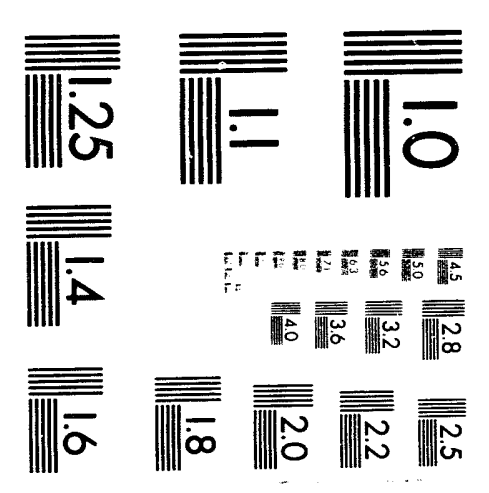



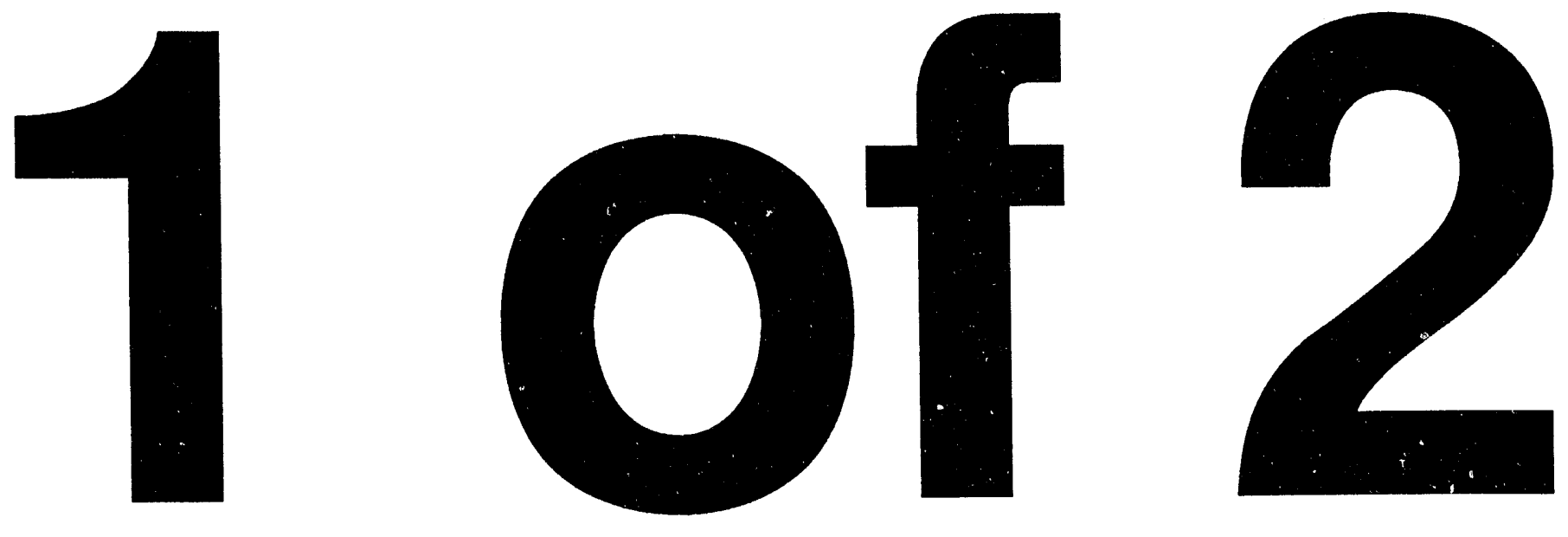
Progress Report for

\title{
Collisional and Chaotic Transport of Energetic Particles in Toroidal Plasma
}

John R. Cary, Principal Investigator

Svetlana G. Shasharina, Co-Investigator

Department of Astrophysical, Planetary, and Atmospheric Sciences

University of Colorado

Boulder, CO 80309-0391

March, 1993

\begin{abstract}
We have made progress in four general areas of confinement plasma physics is proposed. (1) We developed an accurate theory for the trapping/detrapping process in tokamaks with ripple and stellarators. (2) We determined the alpha particle distribution in the situation where both collisions and collisionless chaotic (ripple) transport are present. (3) We investigated transport of the toroidally trapped particles in rippled tokamaks with up-down asymmetry.
\end{abstract}

\section{DISCLAIMER}

\begin{abstract}
This report was prepared as an account of work sponsored by an agency of the United States Government. Neither the United States Government nor any agency thureof, nor any of their employees, makes any warranty, express or implied, or assumes any legal liability or responsibility for the accuracy, completeness, or usefulness of any information, apparatus, product, or process disclosed, or represents that its use would not infringe privately owned rights. Reference herein to any specific commercial product, process, or service by trade name, trademark, manufacturer, or otherwise does not necessarily constitute or imply its endorsement, recommendation, or favoring by the United States Government or any agency thereof. The views and opinions of authors expressed herein do not necessarily state or reflect those of the United States Government or any agency thereof.
\end{abstract}




\section{Table of Contents}

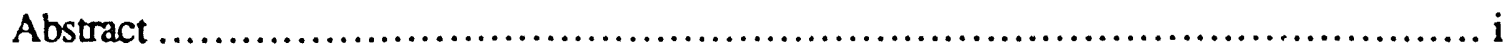

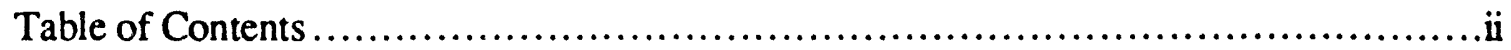

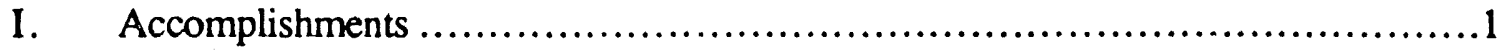

II. Trapping probability in asymmetric tori ......................................

III. Stochastic loss of alphas in tokamak............................................6

IV. Transport in tokamak with vertically asymmetric ripple $\ldots \ldots \ldots \ldots \ldots \ldots \ldots \ldots \ldots \ldots . . .8$

V. References .......................................................... 10

Appendix A. Probability of Orbit Transition in Asymmetric Toroidal Plasma

Appendix B. Ripple Induced Stochastic Loss of Alphas in a Tokamaks

Appendix C. Transport in Non-axisymmetric Tori with Up-Down Asymmetry 
I.

Accomplishments

Research in three general areas of confinement plasma physics was performed. The main effort was to reexamine the collisionless motion of the particles in asymnretric toroidal devices to be abie to formulate the boundary conditions for the distribution function at low collision frequency. Then, we investigated the transport of suprathermal particles (i.e. alpha particles) in the regime where the effects of both collisions and collisionless chaotic transport were taken into account. In addition, we study new transport phenomenae related to up-down asymmetry of the ripple in tokamak.

Studies of transport in asymmetric toroidal devices, e.g. stellarators and tokamaks with ripple, in the low-collisionality regime have been a subject of intense attention in recent years. These investigations are necessary for the understanding of fusion reactors, which must operate at high temperature, where the collision frequency for the particles of the bulk plasma is small. These investigations are also important for smaller experimental devices with neutral beam injected particles.

To carry out the study of the low-collisional transport, we first need to investigate the collisionless motion of the particles in the magnetic fields of the rippled tokamak and stellarator. This motion is not trivial because of the existence of the transitioning particles. ${ }^{1-4}$ These particles make transitions between the locally trapped state and the toroidally trapped state even without collisions. Since these particles can have large radial displacements from the magnetic surfaces, these particles contribute significantly to transport. Hence, an accurate transport theory, either analytical or numerical, must include the evaluation of the trapping probability, the fraction of a toroidally trapped phase uniform distribution that makes a transition to the trapped state at the end of the bounce motion.

In Sec. II, we present a new calculation of the transition probability. The original motivation for re-examining the transition probability calculations was our numerical work 
which showed that previous calculations ${ }^{1-4}$ of the transition probability are inaccurate for realistic parameters. Our new method differs from previous by using a more accurate model of the field variation near the transition and by including in lowest order the poloidal motion due to finite rotational transform.

Another area of our interest is related to alpha particles. The confinement of these alphas is critical to the development of fusion reactors. Our goal here is to include both collisional and collisionless mechanisms of the losses into Fokker-Planck equation. The collisional mechanism is slowing down due to friction on the background electrons and change of the pithch angle due to scattering off the background ions. ${ }^{5,6}$ The collisionless loss occurs on a time scale much shorter then any of the collisional times and is related to the dynamic stochasticity 7,8 of the orbits arising due to magnetic ripple. That is why the stochastic losses are accounted for by assuming that all the stochastic particles are lost immediately. We present the solution of the Fokker Plank equation for the supra thermal particles in Sec.III.

Any breaking of symmetry of the magnetic field should be thoroughly investigated, as it might lead to an additional loss channel and affect the confining properties of the device. That is why we investigate the neoclassical transport associated with up-down asymmetry of the magnetic field in tokamaks and stellarators. Accounting for this asymmetry is important for the devices with vertically displaced magnetic axes 9,10 or with a single-null divertor. The asymmetry manifests itself in different magnitudes of the ripple or helical perturbation in the up and down parts of the magnetic surface. Due to this difference, the toroidally trapped particles acquire a net radial drift. In Sec.IV we study the collisionless dynamics of the particles in such configurations as well as solutions of the kinetic equation. 
II. Trapping probability in asymmetric tori

To investigate the motion of the particles in magnetic confinement devices, tokamak and stellarator, we use the standard model, in which the magnetic field strength has the form,

$$
\left.B=B_{0}\left[1-\varepsilon_{\mathrm{l}}(\psi) \cos (\theta)-\varepsilon_{\mathrm{r}}(\psi) \cos (\mathrm{N} \phi-l \theta)\right)\right]
$$

The numbers $l$ and $\mathrm{N}$ are the poloidal and toroidal mode numbers for a stellarator, while for a tokamak $l=0 ; \psi, \theta, \phi$ are traditional Boozer coordinates. ${ }^{2}$

There are two types of trapped particle orbits, if the drift to neighboring field lines is neglected: the toroidally trapped and the locally trapped particles. The inclusion of drifts allows the toroidally trapped particles to become locally trapped. The slow change of $\psi$ and $\theta$ causes the ripple amplitude and toroidicity to change, while the slow change of $\theta$ causes the phase between the fast and slow components of the magnetic field to change. Due to these effects, a toroidally trapped particle, after passing over the last local maximum near its bounce point, may be trapped by that last maximum. Conversely, a locally trapped particle may result in the decrease of the local maximum, thus allowing the particle to escape the local magnetic well.

We define the trapping probability, $\mathrm{P}$, as a ratio of the flux of the particles trapped into the magnetic well to the total flux reflecting between the points, $\phi_{0}$ and $\phi_{1}$, which are the coordinares of the maxima of the well. We show that $\mathrm{P}$ can be represented as

$$
P_{r}=\left[E_{0}, Y_{0}\right] /\left\{\Delta E_{x}(1-l+/ N)+\frac{1}{2}\left[E_{1}, Y_{1}\right]\right\},
$$

where

$$
Y_{0} \equiv \oint_{E=E_{0}} v_{\varphi} d \varphi,
$$




$$
\begin{gathered}
\mathrm{Y}_{1}=2 \int_{\varphi_{0}}^{\varphi_{1}} \mathrm{~d} \varphi v_{\varphi}, \\
\Delta \mathrm{E}_{\mathrm{x}}=\mathrm{E}_{1}-\mathrm{E}_{0} .
\end{gathered}
$$

Here, $E_{0}$ and $E_{1}$ are the values of energy on the local maxima $\phi_{0}$ and $\phi_{1}$, and $v_{\varphi}=u B_{0} / B$ with $\mathrm{u}$ being the parallel velocity. The Poisson bracket used in Eq.(3) is defined as

$$
[a, b]=\frac{\partial a}{\partial \psi} \frac{\partial b}{\partial \theta}-\frac{\partial a}{\partial \theta} \frac{\partial b}{\partial \psi} .
$$

The main novelties in Eq.(2) in comparison with most of the prevous papers are: (1) we include the additional flux of the particles arising due to drift which also contributes to the total incoming flux (the denominator), (2) we include the correct Lamé coefficient, $B_{0} / B$, into the element of the length, and (3) we distinguish between the values $E_{0}$ and $E_{1}$ which are different due to the finite rotational transfonn. The rotational transform implies that, on the magnetic field line and, hence, in Eq.(2), the poloidal and toroidal angles are related by

$$
\theta=\theta_{0}++\phi
$$

To calculate the integrals in Eqs.(3) and (4) we model the magnetic field, Eq.(1), along the field line by

$$
\mathrm{B} / \mathrm{B}_{0}=\left\{\begin{array}{cl}
1-\varepsilon_{\mathrm{t}} \cos \left(\theta_{0}\right)-\Delta_{0} \cos \left(\mathrm{k}_{0}\left(\phi-\phi_{\mathrm{mn}}\right)\right) & \text { for } \phi_{0}<\phi<\phi_{\mathrm{m} n} \\
1-\varepsilon_{\mathrm{t}} \cos \left(\theta_{0}\right)-\Delta_{1} \cos \left(\mathrm{k}_{1}\left(\phi-\phi_{\mathrm{mn}}\right)\right) & \text { for } \phi_{\mathrm{mn}}<\phi<\phi_{1}
\end{array}\right. \text {, }
$$

where $\Delta_{0}\left(\Delta_{1}\right)$ is the height of the left (right) maximum of the magnetic field relative to the minimum achieved at $\phi_{m n}$, and $k_{i}$ is the contraction factor, $k_{i}=\pi /\left(\phi_{i}-\phi_{m n}\right)$. Applying this model to Eqs. (2)-(4), we obtain analytic expressions for $Y_{0}$ and $Y_{1}$ and we obtain the trapping probability, $\mathrm{P}$, in terms of elliptic integrals. 
We compare the trapping probability obtained by use of the adiabatic theory with direct numerical integration of the guiding center equations. The trajectories are started at $\theta=0$ for various values of $\varphi$ with fixed initial value of the energy and the first-ordercorrected adiabatic invariant. We determine the range of initial values of $\varphi$ for which trajectories reflect or become trapped. Ultimately we find two initial values, $\varphi_{\mathrm{T} 1}$ and $\varphi_{\mathrm{T} 2}$, such that trajectories launched between $\varphi_{\mathrm{T} 1}$ and $\varphi_{\mathrm{T} 2}$ (modulo $2 \pi / N$ ) become trapped, while trajectories launched between $\varphi_{\mathrm{T} 2}$ and $\varphi_{\mathrm{T} 1}$ (modulo $\left.2 \pi / N\right)$ reflect. The numerical trapping probability is then evaluated as a ratio of the fluxes limited by $\varphi_{\mathrm{T} 1}$ and $\varphi_{\mathrm{T} 2}$ to the flux in full period.

For the case depicted on Fig. 9 of App. A, we use parameters which are ATF-like: $\delta_{\mathrm{r}}=0.21, \delta_{\mathrm{t}}=0.14, \varepsilon_{\mathrm{r}}=\delta_{\mathrm{r}} \rho^{2}$ and $\varepsilon_{\mathrm{t}}=\delta_{\mathrm{t}} \rho$. where $\rho$ is the minor radius, $t=0.35+0.55 \rho^{2}$. The electrostatic potential is assumed to have parabolic form: $\Phi=\Phi_{0} \rho^{2}$. The energy parameters, $\gamma_{\mu} \equiv \mu \mathrm{B}_{0} / \mathrm{m}(\Omega \mathrm{a})^{2}, \gamma_{\mathrm{E}} \equiv \mathrm{E} / \mathrm{m}(\Omega \mathrm{a})^{2}$, and $\gamma_{\Phi} \equiv \mathrm{q} \Phi_{0} / \mathrm{m}(\Omega \mathrm{a})^{2}$, are $\gamma_{\mu}=1.81 \times 10^{-4}, \gamma_{E}=3 \times 10^{-4}$, and $\gamma_{\Phi}=2 \times 10^{-4}$ with $\mathrm{N}=12$

Our numerical analysis shows that the new approach to evaluate the trapping probability, as well as the model of the magnetic field, the adiabatic invariant are superior to those used in the previous papers because it overall give the best prediction for the trapping probability. 
III. Stochastic loss of alphas in tokamak

In the presence of asymmetric magnetic fields, such as ripple, the trajectories of fast particles can be chaotic. These chaos arises due to resonance between the toroidal precession and the bounce frequency. The stochastic diffusion rate exceeds other mechanisms by order of magnitude. Hence, the determination of the fast particle loss due to stochasticity is crucial for knowing whether alphas can be well confined. We reexamined the motion of alpha particles in rippled magnetic fields in order to determine the distribution and heat deposition of fusion produced alphas in the presence of both chaotic losses and collisional redistribution of pitch angle and energy.

To model the fast chaotic losses we impose the boundary condition that the particle distribution vanishes in the stochastic region of the velocity space. This region is specified by the Goldston-White-Boozer criterion,

$$
\xi<\xi_{\mathrm{t}}, \quad \mathrm{v}>\mathrm{v}_{\mathrm{s}},
$$

with

$$
v_{S}=\frac{\Omega}{2 \varepsilon_{r}}\left(\frac{\varepsilon_{t}}{\pi N q}\right)^{3 / 2} \frac{1}{d q / d r},
$$

where $q=1 / t$ and $\xi=\left[1-2 \mu B_{o} / v^{2}\right]^{1 / 2}$, and $r$ is the minor radius of the torus. Eqs.(9) and (10) mean that the particle should be toroidally trapped and sufficiently fast. We impose these boundary conditions to the stationary drift kinetic equation,

$$
\frac{u \varepsilon_{t}}{q r}=C p(f)+C_{v}(f)+S_{0} \delta\left(v-v_{0}\right)
$$

with the Lorentz collision operator, $C_{P}(f)$, slowing down term, ${ }^{5,6} C_{v}(f)$, and the source, $\mathrm{S}_{0} \delta\left(\mathrm{v}-\mathrm{v}_{0}\right)$ 
We assume that the radial width of the banana orbit is small. We apply Cordey's procedure of expanding in the ratio of the collision frequency to the bounce frequency. Then the lowest order term, $\mathrm{f}_{0}$, can be found by use of the solubility condition and separable expansion, $f_{0}=\sum_{n} a_{n}(v) C_{n}(\xi)$, in the eigen-functions of the operator $C_{P}(f) / u$ averaged along the trajectory. We then evaluate the relative flux of the particles into stochastic region gives the relative stochastic loss of alphas,

$$
\gamma_{s t}=-<\int_{M} C_{p}\left(f_{o}\right) d^{3} v>/\left(4 p v_{0}^{2} S_{0}\right)
$$

where $M$ is the the velocity space with $v<v_{0}$ and without stochastic loss cone, and the angular brackets denote the magnetic surface averaging.

For the "typical" case, i.e. ITER design, we found that the alpha particle loss constitutes approximately $60 \%$ of all the particles and the energy loss is up to $15 \%$. 


\section{Transport in Non-axisymmetric Tori with Up-Down Asymmetry}

The most important classical channel of the particle loss in toroidal magnetic systems is the ripple diffusion of the toroidally trapped particles. 1,7,8 It arises due to distortion of the axial symmetry of the torus by the finite perturbation of the toroidal magnetic field. Hence, the ripple diffusion has been a subject of intense studies for the ideal configurations as well as for concrete devices.

The best solution of the problem is to diminish the toroidal asymmetry. But decreasing the ripple magnitude is quite expensive, because it requires the volume of the coils to be increased. That is why studies were aimed mostly to estimate the critical magnitude of ripple in order to find the balance between allowed losses and a reasonable design of the device. It is not only the loss value which should be known. The distribution of the particle and energy flux on the chamber walls is also very important. Here, the influence of the geometry and the features of the orbits come into play. Although the main loss of bulk plasma is expected to be governed by anomalous mechanisms, the classical loss of high energy particles (alpha-particles, NBI-ions, tail particles) is comparable with the anomalous one.

In this regards, any new breaking of the symmetry of the magnetic field should be thoroughly investigated, as it might lead to an additional loss channel and affect the confining properties of the device.

In the present paper we investigate the neoclassical transport associated with updown asymmetry of the magnetic field in tokamaks and stellarators. In stellarators, the ripple magnitude is usually considered to be some function of the minor radius. In several cases, helical modulation dependent on the poloidal angle was studied, ${ }^{11}$ but the up-down symmetry was always implied both for stellarators and tokamaks. However this is not always the case in the real experiments. Substantial up-down asymmetry can arise due to 
even rather small vertical displacement of plasma with respect to the center of the chamber. 9,10 Besides, all magnetic configurations with a single null divertor are naturally asymmetric from the very beginning. In such cases, the depth of the local magnetic wells, $\Delta$, is different in the upper and lower parts of the magnetic surfaces. The value of this asymmetry can be of order unity, $\left(\Delta^{+}-\Delta^{-}\right) /\left(\Delta^{+}+\Delta^{-}\right) \approx 1$. In these magnetic configurations, the toroidally trapped particles - those with reflection points located in the region where the local magnetic wells exist - acquire a net radial velocity, which can be estimated from Liouville's theorem,

$$
\dot{r}_{\mathrm{t}} \approx v_{\mathrm{d}} \sqrt{\frac{\varepsilon_{\mathrm{r}}}{\varepsilon_{\mathrm{t}}}},
$$

where $v_{d}$ is the gradient drift velocity. This velocity could lead to an additional transport. This is the case at low collisionality, when the presence of the physical boundaries is essential and the losses due to new net drift motion are convective and quite rapid. The particles are lost at a rate

$$
\frac{1}{\tau} \approx \min \left\{\frac{\mathrm{a}}{\dot{\mathrm{r}}_{\mathrm{t}}}, \frac{\mathrm{a}}{\mathrm{v}_{\mathrm{d}}}\right\} .
$$

where $\mathrm{a}$ is the minor radius of the torus.

The situation is different at higher collisionality, when the radial displacement of the particles between the collisions is small. In this case, we solve the bounce-averaged kinetic equation,

$$
\begin{aligned}
& \langle\dot{\mathrm{r}}\rangle^{\prime} \frac{\partial \mathrm{F}^{\mathrm{t}}}{\partial \mathrm{r}}+\langle\dot{\beta}\rangle^{\mathrm{t}} \frac{\partial \mathrm{F}^{\prime}}{\partial \beta}=\frac{v}{\mathrm{~B}_{0} \partial \mathrm{J}^{\mathrm{t}} / \partial \mathrm{E}} \frac{\partial}{\partial \mu} \mu \mathrm{I}^{\prime} \frac{\partial \mathrm{F}^{\prime}}{\partial \mu}, \\
& \langle\dot{\mathrm{r}}\rangle^{\mathrm{r}} \frac{\partial \mathrm{F}^{\mathrm{r}}}{\partial \mathrm{r}}+\langle\dot{\beta}\rangle^{\mathrm{r}} \frac{\partial \mathrm{F}^{\mathrm{r}}}{\partial \beta}=\frac{v}{\mathrm{~B}_{0} \partial \mathrm{J}^{\mathrm{r}} / \partial \mathrm{E}} \frac{\partial}{\partial \mu} \mu \mathrm{I}^{\mathrm{r}} \frac{\partial \mathrm{F}^{\mathrm{r}}}{\partial \mu},
\end{aligned}
$$

where 


$$
\begin{aligned}
& \langle\dot{r}\rangle^{\prime}=\frac{1}{r \Omega \partial J^{t} / \partial E}\left(\frac{\partial J^{t}}{\partial \beta}-\delta(Q) \frac{\partial Q}{\partial \beta} J_{s}^{s}\right) \equiv \dot{r}^{t}+\dot{r}_{b}^{t}, \\
& \langle\dot{\beta}\rangle^{\prime}=-\frac{1}{r \Omega \partial J^{t} / \partial E}\left(\frac{\partial J^{t}}{\partial r}-\delta(Q) \frac{\partial Q}{\partial r} J_{s}^{r}\right) \equiv \dot{\beta}^{t}+\dot{\beta}_{b}^{t},
\end{aligned}
$$

are the average components of the drift velocity,

$$
\begin{aligned}
& J=\oint R_{0}^{2} \dot{\phi} d \phi=\oint R_{0} \frac{u B_{0}}{B} d \phi, \\
& I=\oint \frac{B R_{0}^{2}}{B_{0}} \dot{\phi} d \phi=R_{0} \oint u d \phi, \\
& T_{b}=\oint \frac{d \phi}{\dot{\phi}}=\frac{\partial J}{\partial E} .
\end{aligned}
$$

where we omit the indices distinguishing the toroidally (" $t$ ") and locally trapped ("r") states and the function $Q$ determines the boundary between these states, so that the separatrix is given by,

$$
Q(r, \beta, E, \mu)=0
$$

The rigorous analysis of the bounce-averaged kinetic equation (App. C) shows that the additional diffusive flux of the toroidally trapped particles, which is produced by the $\beta$ independent part of the distribution function, is totally compensated by the change in the flux of the locally trapped particles, so that the main transport remains unchanged. The remaining contribution to the diffusion in $1 / \mathrm{V}$-regime of the toroidally trapped particles coming from $\tilde{\mathrm{F}}^{\prime}$ is almost the same for the up-down symmetric and asymmetric tori. It has been investigated before ${ }^{11}$ and is related to $1 / \mathrm{N}$-regime of the locally trapped particles by the relation, $D_{t}^{\text {sym }} / D_{r}^{\text {sym }} \approx \frac{1}{(q N)^{2}} \sqrt{\frac{\varepsilon_{t}}{\varepsilon_{r}}}$. Although, note that some new effects due to the asymmetry, which do not change the order of the fluxes, can be expected in the results obtained before ${ }^{11}$ since we should include the general (up-down asymmetric) dependence 
of $\varepsilon_{\mathrm{r}}$ on the poloidal angle. It will give new terms for $\frac{\partial J^{\mathrm{t}, \mathrm{r}}}{\partial \beta}$ with a relative order $\frac{\partial \varepsilon_{\mathrm{r}} / \partial \beta}{\varepsilon_{\mathrm{t}}}$ in comparison with the conventional ones. 
V.

\section{References}

1 R.J. Goldston and H.H Towner, J. Plasma Phys. 26, 283 (1981).

2 J.R. Cary, C.L. Hedrick, and J.S. Tolliver, Phys. Fluids. 31, 1586 (1988).

3 V.P. Nagornij and V.A. Yavorskij, Sov.J. Plasma Phys. 15, 309 (1989).

4 L.M. Kovrizhnykh and S.G. Shasharina, Nucl. Fusion 30, 453 (1990).

5 J.D. Cordey, Nucl. Fusion 16, 199 (1976).

6 C.T. Hsu, P.J. Catto, and D.J. Sigmar, Phys. Fluids B2, 280 (1990).

7 R. J. Goldston, R. B. White, and A. H. Boozer, Phys. Rev. Lett. 47, 647 (1981).

8 R. B. White and H. E. Mynick, Phys. Fluids B 1, 980 (1989).

9 D.P. Schlissel, T.H. Osborne, J.C. DeBoo, J.R. Ferron, E.A. Lazarus, T.S. Taylor, Nuclear Fusion 32, 689 (1992).

10 R.B. Bengston, A. Ouroua, A.G. Meigs, Bulletin of the American Physical Society, 34, No 9, paper $8 \mathrm{~S} 3$.

11 L.M.Kovrizhnykh, Nuclear Fusion 24, 851 (1984). 
Probability of orbit transition in asymmetric toroidal plasma

\author{
John R. Cary and S. G. Shasharina*
}

Department of Astrophysical, Planetary, and Atmospheric Sciences

and Department of Physics

University of Colorado

Boulder, CO 80309-0391

USA

\begin{abstract}
Trapped particles in stellarators and tokamaks can be either locally trapped in the ripple wells or toroidally trapped by the $1 / R$ variation of the magnetic field strength. Transitioning particles make transitions back and forth between these two states. The transition probability gives the relative rate of toroidally trapped particles to become locally trapped. In this paper the transition probably is uniquely defined, and an inproved analytical calculation of the transition probability is given. It is then calculated numerically by following the full guiding-center dynamics. Comparison with the numerical results shows that the present calculation is more accurate than those previously presented.
\end{abstract}

PACS nos. 03.20.+i, 28.52.Av, 52.20.Dq

(Received: 
Appendix A. Probability of Orbit Transition in Asymmetric Toroidal Plasma 
I .

Introduction

Studies of transport in asymmetric toroidal devices, e.g. stellarators and tokamaks with ripple, in the low-collisionality regime have been a subject of intense attention in recent years ${ }^{1-4}$. These investigations are necessary for the understanding of fusion reactors, which must operate at high temperature, where the collision frequency for the particles of the bulk plasma is small. Even in smaller experimental devices such low-collisionfrequency studies are important for developing an understanding of transport and confinement of supra-thermal ions, such as alpha particles and neutral beam injected particles.

Unanimity of understanding in this area has not yet been achieved. As noted in Refs. 1 and 2, several contradictory scalings for the neoclassical diffusion coefficient in asymmetric tori have been proposed. These discrepancies arise due to the various choices of the boundary conditions for the collisionless distribution function on the phase-space boundary between two types of motion: toroidally-trapped and locally-trapped. Toroidally trapped orbits are analogous to the banana orbits found in axisymmetric devices. The particles are reflected by the increase of the magnetic field due to moving to smaller major radius. Locally trapped particles are trapped between the local magnetic maxima produced by the magnetic coils.

The choice of the boundary condition is complicated because of the existence of transitioning particles. ${ }^{1-8}$ These particles make transitions between the locally trapped state and the toroidally-trapped state even without collisions. Since these particles can have large radial displacements from the magnetic surfaces, these particles contribute significantly to transport. Hence, an accurate transport theory, either analytical or numerical, must include the proper boundary conditions for the transitioning part of phase space. 
The particle motion is governed by the guiding-center equations of motion, because for all particles the gyroradius is small compared with scale length of the magnetic field. However, the guiding-center equations are analyzed only with difficulty in asymmetric configurations. Their use in determining the distribution function ${ }^{9}$ requires the solution of a drift-kinetic equation involving both the toroidal and poloidal angles as well as the velocity space. This is significantly more complicated than the axisymetric case, in which dependence on the toroidal angle is absent. As a result, such calculations are difficult in the very low collision frequency limit. In addition, the use of guiding-center equations for Monte Carlo simulations is computationally intensive, due to the need to follow particles on two quite different time scales, the time to traverse or bounce in a local ripple well, and the drift time.

For this reason, a "bounce-averaged" approach is advantageous in neoclassical theory. This approach is based upon the existing time-scale hierarchy. After elimination of the gyrofrequency with guiding-center theory, the highest frequency in the system is the local bounce-frequency, the inverse of the time to bounce in or traverse a local ripple well. An ordering based on this time-scale disparity permits an adiabatic theory. ${ }^{10}$ This adiabatic theory finds a transformation to local-bounce adiabatic variables, in which the toroidal angle is absent. One of the variables of this adiabatic theory is the local-bounce adiabatic invariant, J. This invariant is conserved to all orders ${ }^{11}$ in the adiabatic parameter $\varepsilon_{\mathrm{a}}$, essentially the time-scale ratio. This statement means that one can construct an asymptotic series, $\mathrm{J}=\mathrm{J}_{0}+\varepsilon_{\mathrm{a}} \mathrm{J}_{1}+\ldots$, whose time derivative vanishes to all orders in the adiabatic parameter. Note, that the non-asymptotic term of order $\exp \left(-1 / \varepsilon_{a}\right)$ is not represented in this formalism. In the adiabatic variables, the poloidal (drift and rotational transform) motion decouples from the local-bounce motion, so the rapid time-scale has been eliminated from the equations of motion. 
The adiabatic phase, i.e. the variable conjugate to $\mathrm{J}$ which locates the particle on a $\mathrm{J}$. contour, for the ripple bounce motion increases at a rate which depends on $J$ and the poloidal location. Though calculable, it is simplifying to ignore this adiabatic phase. For most of the motion this is possible, since the phase does not affect the poloidal motion of the particles. However, the transition process does depend on the phase. But, since the phase is uniformly distributed due to both the collisionless dynamics (see Sec. II) and collisional effects, if the evolution of a distribution is desired, it is necessary to know only the transition probability - the fraction of a toroidally trapped phase uniform distribution that makes a transition to the trapped state at the end of the bounce motion.

The transition probability has been evaluated in a number of papers. ${ }^{1,2,5-10}$ Several approaches, based on somewhat different assumptions, were used. Since this quantity is very important in the investigation of transport in the low-collisionality regime, a comparison of these results and their accuracy relative to fully numerical guiding center integrations is needed. This is the goal of this paper.

We begin in the following section with a general discussion of transitioning orbits in asymmetric toroidal devices. We recall the reasons for the existence of transitioning orbits, and the Hamiltonian guiding-center equations of motion. The theory of integrable Hamiltonian systems allows us to define a unique transition probability as the relative flux of trajectories that become trapped.

In Sec. III we discuss the local-bounce adiabatic theory. Results of the general theory of separatrix crossing show that for numerical experiments it is necessary to know the local-bounce adiabatic invariant theory through first order in the adiabatic parameter. Next we review the previous analysis of the lowest-order adiabatic invariant. We finish this section with a calculation of the first-order corrections to the adiabatic invariant. This 
calculation is quite general; it applies to any Hamiltonian system of two degrees of freedom having a time-scale disparity between the canonical pairs

In Sec. IV we present a new calculation of the transition probability. The original motivation for re-examining the transition probability calculations was our numerical work, discussed below, which showed that previous calculations $1,7,8,10$ of the transition probability are inaccurate for realistic parameters. Our new method differs from previous by using a more accurate model of the field variation near the transition and by including in lowest order the poloidal motion due to finite rotational transform.

In Sec. $V$ we compare the various transition probabilities with direct numerical integrations. Our goal is to see whether adiabatic theory, which relies on the time-scale separation, is sufficiently accurate for realistic configurations. In this section we also make head-to-head comparisons of the published analytic results for the transition probability. We show that our new method of calculating the transition probability is reasonably accurate for typical parameter values and that it is an improvement over previous results.

In the last section we present a summary and a discussion of needed research. The two appendices contain information on particulars of the standard stellarator model. Appendix $\mathbf{A}$ is a discussion of the near-separatrix limits of the lowest-order adiabatic invariants. In App. B the corrections to the adiabatic invariant are evaluated for the standard stellarator model. 
II.

Background

In this section, we describe the transitioning orbits that occur in stellarators and tokamaks with ripple. We first discuss the different types of trajectories in such a system. We note how drifts can cause particles to make the transition from one state to another. Next we review general aspects of integrable motion and show how it is applied to adiabatic theory. Finally, we discuss the modifications for cases where transitions are possible.

\section{A. Types of orbits in stellarators and rippled tokamaks}

We use Boozer coordinates ${ }^{12}$ (flux variable: $\psi$, poloidal angle: $\theta$, toroidal angle: $\varphi$ ), in which the magnetic field,

$$
\text { B }=\nabla \psi \times \nabla \theta+t(\psi) \nabla \varphi \times \nabla \psi=B_{\psi}(\psi, \theta, \varphi) \nabla \psi+B_{\theta}(\psi) \nabla \theta+B_{\varphi}(\psi) \nabla \varphi
$$

has constant Clebsch representation components and angular covariant components. The quantity $t=1 / q$ is the rotational transform of the field lines, the inverse of the safety factor q. In these coordinates, the standard model (Ref. 10, App. B) is one in which the magnetic field strength has the form,

$$
B=B_{0}\left[1-\varepsilon_{t}(\psi) \cos (\theta)-\varepsilon_{r}(\psi) \cos (\chi)\right]
$$

where

$$
\chi \equiv N \varphi \cdot l \theta .
$$

This field strength varies due to toroidicity and a single ripple harmonic. The numbers $l$ and $\mathbf{N}$ are the poloidal and toroidal mode numbers for a stellarator, while for a tokamak $l=0$. 
To lowest order particles move along field lines. Hence, it is convenient to introduce an angle,

$$
\beta \equiv \theta-t \varphi,
$$

that is constant along a field line and linear in the angles $\theta$ and $\varphi$. Thus, along a field line the magnetic field has the form,

$$
B=B_{0}\left[1-\varepsilon_{l}(\psi) \cos (\theta)-\varepsilon_{r}(\psi) \cos ((N q-l)(\theta-\beta)-l \beta)\right]
$$

Differentiation of Eq. (3) along a field line (i.e., with respect to $\theta$ at constant $\beta$ ) gives the condition,

$$
\varepsilon_{\mathrm{q}} \sin (\theta)=-(\mathrm{Nq}-l) \varepsilon_{\mathrm{r}} \sin (\chi)
$$

for the existence of a local magnetic minimum and, therefore, a local magnetic well. Hence, when

$$
\alpha \equiv \frac{\varepsilon_{\mathrm{I}}|\sin (\theta)|}{(\mathrm{Nq}-l) \varepsilon_{\mathrm{r}}} \leq 1
$$

is satisfied, there exist magnetic wells which can trap particles locally, i.e., within one ripple period. This condition is typically satisfied for stellarators. A sample magnetic field variation is shown in Fig. 1. For most of tokamaks this condition (7) is usually satisfied only near the midplane, but it may be satisfied away from the midplane if the ripple is large.

To lowest onder in guiding-center theory, a particle remains on a field line. In this case one can distinguish two types of trapped particle orbits (cf Fig. 1). Toroidally trapped particles are those which move over many ripple periods. They have sufficient energy to pass over one or more of the local magnetic maxima on that field line. The locally trapped 
particles are trapped in a single ripple period between two magnetic maxima on that field line.

Toroidally trapped particles can become locally trapped due to magnetic drifts and the $\mathrm{E} \times \mathrm{B}$ drift due to the potential $\Phi(\psi)$ that arises from the ambipolarity constraint. Drifts cause $\psi$ and $\beta$ to change slowly. The slow change of $\psi$ causes the ripple amplitude and toroidicity to change, while the slow change of $\beta$ causes the phase between the fast and slow components of the magnetic field in Fig. 1 to change. Due to either or both of these effects, the particle may drift to a different field line on which the value of the maximum is greater. Thus, after passing over the last local maximum near its bounce point on one field line, a toroidally trapped particle may be trapped by that last maximum after reflection and drift to a different field line. This process is illustrated in Fig. 2. Conversely, a locally trapped particle may result in the decrease of the local maximum, thus allowing the particle to escape the local magnetic well.

Indeed, this typically happens, so that all toroidally trapped particles eventually become locally trapped. However, there are some locally deeply trapped particles that never drift to a field line with sufficiently small local maxima to detrap. Thus, there are three types of particles: the superbananas, which stay locally trapped indefinitely, the passing particles, are never reflected by the magnetic field variations, and the transitioning particles, which have one portion of their trajectories in the toroidally trapped state and another in the locally trapped state.

\section{B . Hamiltonian guiding-center theory}

Hamiltonian theory allows one to analyze the just discussed orbits by adiabatic theory and so provides the reduction to a system with fewer degrees of freedom. It also allows us to define the trapping probability unambiguously, the goal of this paper. Here we recall a few features of such systems that we will need to use in the adiabatic analysis. 
The Hamiltonian function gives the differential equations for the dynamics of a Hamiltonian system. These are derived from Hamilton's equations,

$$
\dot{q}_{i}=\frac{\partial h}{\partial p_{i}}
$$

and

$$
\dot{p}_{i}=-\frac{\partial h}{\partial q_{i}}
$$

Equivalently, they come from the phase-space Lagrangian,

$$
\mathrm{L}_{\phi}=\sum_{\mathrm{i}} \mathrm{p}_{\mathrm{i}} \dot{\mathrm{q}}_{\mathrm{i}}-\mathrm{h}
$$

Independent variation of this Lagrangian with respect to the phase-space variables $q$ and $p$ gives Hamilton's equations (8). An advantage of the phase-space Lagrangian formalism is that it allows arbitrary (noncanonical) transformations of the $2 \mathrm{~N}$ phase-space variables $z \equiv\left(q_{1}, \ldots, q_{N}, p_{1}, \ldots, p_{N}\right)$

Of particular importance here is that there is a phase-space Lagrangian for guidingcenter motion. This Lagrangian is

$$
L_{\varepsilon=}=(e A / c+m u b) \cdot \dot{X}-h,
$$

where

$$
h=\frac{1}{2} m u^{2}+V(X, t)
$$

is still known as the Hamiltonian, and

$$
V(X, t) \equiv \mu B(X, t)+e \Phi(X, t)
$$


is the potential energy. This Lagrangian is straightforwardly expressed in terms of physical variables, such as the parallel velocity $u$, the guiding-center position $X$, and the magnetic moment $\mu$. The equations of motion are the Euler-Lagrange equations obtained by variation with respect to the variables $(\mathbf{X}, u)$. In the phase-space Lagrangian the time derivatives of the variables occur linearly. This is seen to hold in particular for both Lagrangians (9) and (10a). For the Lagrangian (10a) there are four phase-space variables $(\mathbf{X}, \mathrm{u})$, so this is a system of two degrees of freedom.

The phase-space Lagrangian (10a) is not in the canonical form (9). Canonical form means that the time derivatives of only half (in this case two) of the phase-space variables appear in the Lagrangian, and that the coefficients of these time derivatives, which must appear linearly as noted above, are the other two variables. General canonical coordinates do exist, ${ }^{13}$ but they are often not convenient because of the need to solve a differential equation to find them. However, for nested magnetic surfaces, the case of interest here, convenient coordinates do exist.

The vector magnetic potential for systems with nested magnetic surfaces has the form,

$$
A=\psi \nabla \theta+A_{\varphi}(\psi) \nabla \varphi
$$

where $A_{\varphi}$ is related to the rotational transform via

$$
t=-\frac{d A_{\emptyset}}{d \psi} \text {. }
$$

Thus, in these coordinates, the phase-space Lagrangian reduces to

$$
L_{\varphi}=\left(\psi+m u b_{\theta}\right) \dot{\theta}+\left(\frac{e}{c} A_{\varphi}+m u b_{\varphi}\right) \dot{\varphi}+u b_{\psi} \dot{\psi}-h .
$$


Here, $b_{j}$ is the covariant component of the magnetic unit vector, i.e., $b_{\theta}=B_{\theta} / B$. The Euler-Lagrange equations obtained by variation of the Lagrangian (13) with respect to the variables $(\psi, \theta, \varphi, u)$ are the equations of motion.

This Lagrangian is not in canonical form either. It can be put into canonical form ${ }^{14}$ exactly, but we choose to simply drop the terms in $b_{\psi}$ and $b_{\theta}$. This is justified because the quantity $b_{\psi}$ is typically much smaller than $b_{\theta}$, which is smaller than $b_{\varphi}$ by the inverse aspect ratio squared, and $\dot{\psi}$ is much smaller than $\dot{\theta}$ and $\dot{\varphi}$, which are of the same order for rotational transform of order unity. With these approximations, the phase-space Lagrangian has the canonical form,

$$
L_{\phi}=(e \psi / c) \dot{\theta}+\left(e A_{\phi} / c+m u b_{\varphi}\right) \dot{\varphi}-h
$$

Thus, the canonical momentum conjugate to the poloidal angle is, up to a constant factor, the (toroidal) flux variable $\psi$, while the canonical momentum conjugate to the toroidal angle is

$$
p_{\varphi}=e A_{\varphi} / c+m_{\varphi}
$$

In terms of these canonical variables the Hamiltonian is

$$
h=\frac{\left(p_{\varphi} \cdot e A_{\varphi} / c\right)^{2}}{2 m b_{\varphi}^{2}}+V(\psi, \theta, \varphi)
$$

\section{Overview of the reduction of the hamiltonian to integrable form}

The goal of adiabatic theory is to reduce the hamiltonian to integrable form, so that the motion is completely known. In essence, this involves finding an invariant of the motion via adiabatic analysis. This reduction has been carried out through lowest order in 
Ref. 10. Here we review this analysis in order to be able to discuss its improvement in the Sec. III.

In stellarators or rippled tokamaks there is no obvious constant of the motion. However, when $t l / \mathrm{N}$ and $t \varepsilon_{l} l / \mathrm{N} \varepsilon_{r}$ are small, invariants of the motion can be found by an adiabatic theory ${ }^{10}$ based on the fact that to lowest order particles stay at fixed poloidal location. Not even the motion along field lines causes a significant change of the poloidal angle in one ripple period when the rotational transform per field period is small.

The first step of this theory is to separate the particles into three classes. Those with energy, $E<V_{u m}$, less than the local toroidal maximum (at constant $\psi$ and $\theta$ since even the $\theta$-motion is small) of the potential energy, where

$$
V_{\mathrm{tm}}(\psi, \theta) \equiv \mu B_{0}\left[1-\varepsilon_{\mathrm{t}}(\psi) \cos (\theta)+\varepsilon_{\mathrm{r}}(\psi)\right]+\mathrm{e} \Phi(\psi)
$$

are locally trapped. Those satisfying $E>V_{t m}$ are positive passing for $u>0$ and negative passing for $u<0$. For each of these classes of particles there is an adiabatic invariant, and adiabatic theory predicts that the motion stays on the invariant surfaces. These invariant surfaces meet at the transition surface defined by $E=V_{\text {tm }}$. As in Ref. 10 the ripple trapped invariant is denoted $\mathrm{J}_{\boldsymbol{r}}$, while the passing invariants are denoted by $\mathrm{J}_{ \pm^{-}}$. The invariants $\mathrm{J}_{ \pm}$ are to lowest order simply the ripple averages of the toroidal angular momentum.

Figure 3 (calculated using the theory of Ref. 10) shows the poloidal cross-section of the adiabatic invariants for a case without electrostatic potential. The contours of the three distinct types of motion join at the transition curve, $\mathrm{E}=\mathrm{V}_{\mathrm{tm}}$, the heavily drawn partial circle. The object made up by connecting three invariant surfaces touching at the transition curve and revolving in the toroidal angle is called a patched torus. (Patched tori also occur in the motion of particles in the earth's magnetotail. ${ }^{15}$ ) 
The arrows on the contours show the direction of the particle motion. The locally passing particles, inside the transition curve, bounce between the top and bottom parts of the transition curve. This figure shows that the positive passing particles and the locally trapped particles flow into the upper half of the transition curve, and the negative passing particles flow out. Thus, all trajectories in this case eventually become negative passing, so the negative passing state is called the majority state. Upon reaching the lower half of the transition curve the negative passing particles must either reflect, coming out in the positive passing state, or trap, coming out in the locally trapped state. The trapping probability gives the relative frequency of becoming trapped.

At and near transition, where the particles are forced to cross a separatrix, a contour of the Hamiltonian separating topologically distinct types of motion, adiabatic theory breaks down. The theory of separatrix crossing ${ }^{16,17}$ has seen many recent advances. Here we will apply this theory to the motion in rippled toroidal magnetic fields. In separatrix crossing the particular state into which the trajectory passes is determined by the phase of the trajectory. However, it is just such phase information that one would like to be able to ignore, since to keep it requires that the trajectories be followed on the ripple time scale. For this reason, we want to show that there is a natural ensemble which leads to a well defined transition probability.

Separatrix crossing theory also predicts a phase-dependent part of the change of the adiabatic invariant. This means that an ensemble initially having one value of $J$. (for example) becomes spread over a range of values of $\mathrm{J}_{\mathrm{r}}$ and $\mathrm{J}_{+}$after crossing the separatrix. This spreading will be ignored here, since it is of first order in the adiabaticity parameter, which in this case is the ratio of the ripple to the bounce and/or drift time scale. However, that this effect always leads to chaos in the adiabatic limit. ${ }^{18}$ For this reason it was noted that all transitioning particles are chaotic, ${ }^{19}$ and that on long enough time scales the chaos 
due to this phase-dependent change of the adiabatic invariant can cause transport without collisions.

As mentioned above, adiabatic theory gives invariants $J_{r}$ and $J_{ \pm}$for each of the possible states: locally trapped, positive passing, and negative passing. Corresponding to each of these states are also the associated canonically conjugate angles, $\zeta_{r}$ and $\zeta_{ \pm}$(the adiabatic toroidal angle). Perturbation theory gives the transformation from the original coordinates $\left(\psi, \theta, \varphi, p_{\varphi}\right)$ to these new coordinates. The transformation also changes the variables of the poloidal degree of freedom, so that there are new variables $\psi_{j}$ (the adiabatic flux) and $\theta_{j}$ (the adaibatic poloidal angle), where $j=r$ or \pm .

In addition the adiabatic theory ${ }^{10}$ gives the Hamiltonian as a function of these new variables. In fact there is a Hamiltonian $\mathrm{H}_{\mathrm{j}}\left(\psi_{\mathrm{j}}, \theta_{\mathrm{j}}, \mathrm{J}_{\mathrm{j}}\right)$ for each of the possible states. At this point the problem has been reduced to a one-degree-of-freedom system and can be solved by standard methods. For each state the evolution is given by Hamilton's equations,

$$
\dot{\theta}_{j}=\frac{\partial H_{j}}{\partial \psi_{j}}
$$

and

$$
\dot{\psi}_{j}=-\frac{\partial H_{j}}{\partial \theta_{j}}
$$

Alternatively, since $\mathrm{H}_{\mathrm{j}}$ is a constant of the motion, the time to go any range of angle can be found by integrating,

$$
\mathrm{dt}=\mathrm{d} \theta / \frac{\partial \mathrm{H}_{\mathrm{j}}}{\partial \psi_{\mathrm{j}}}\left(\Psi_{\mathrm{j}}, \theta_{\mathrm{j}}, \mathrm{J}_{\mathrm{j}}\right)
$$

in which $\Psi_{j}$ is known from the fact that $\mathrm{H}_{\mathrm{j}}$ is a constant of the motion. 
Use of this formalism for Monte Carlo simulation would involve the following. Equations (18) are used to follow a trajectory until it meets a separatrix. If the particle was in a minority state, it is then put into the majority state, and the integration is continued with the appropriate Hamiltonian. If the particle was in the majority state, then it is put into the trapped state according to the transition probability. Hence, to complete the understanding, we need to discuss the trapping probability.

\section{Definition of the trapping probability}

The previous section we noted how the adiabatic theory could be used to follow particle trajectories as might be done in, for example, a Monte Carlo simulation. The only uncertainty in this process is the transition probability, which answers the question: When a particle in the majority state reaches the transition curve, what fraction of the time does it make a transition to one of the minority states as opposed to the other? To answer such a question, one must first show that there is a unique ensemble. For near-integrable Hamiltonian systems, there is a unique ensemble, one that is uniform in the adiabatic phase over the entire torus. From this it follows that the transition probability equals the fraction of initial adiabatic toroidal angle at constant adiabatic poloidal angle that makes the transition to one state or the other. This definition is not convenient for numerical experiments, but we show that it implies that the transition probability is just the relative flux of particles between two invariant tori on any cut (not necessarily one of constant adiabatic poloidal angle) that make the desired transition.

To make our discussion of the unique ensemble for integrable Hamiltonian systems more concrete, we put our explanation in terms of the well known case of drift orbits in an axisymmetric torus. In this case the Hamiltonian (16) is independent of the toroidal angle $\varphi$, and so the angular momentum $\mathrm{P}_{\varphi}$ is an invariant of the motion. Thus, a trajectory is 
confined to a surface of constant $h$ and $p_{\varphi}$. The cross-sections of these tori are nearly circular for passing particles and of the shape of a banana for trapped particles.

Standard methods provide a canonical transformation to the bounce action $\mathrm{I}_{\mathrm{b}}$, which is constant on contours of the Hamiltonian $h\left(\psi, \theta, p_{\varphi}\right)$. This transformation provides a new bounce angle $\theta_{b}$ conjugate to the bounce action and a new toroidal angle $\zeta$ conjugate to the toroidal angular momentum. In these variables the Hamiltonian $h=H\left(I_{b}, p_{\varphi}\right)$ is a function of only the momentum variables, which are constants of the motion. Hamilton's equations,

$$
\dot{\theta}_{b}=\omega_{b} \equiv \frac{\partial H}{\partial I_{b}}\left(I_{b}, P_{\varphi}\right)
$$

and

$$
\dot{\zeta}=\omega_{\zeta} \equiv \frac{\partial \mathrm{H}}{\partial \mathrm{p}_{\varphi}}\left(\mathrm{I}_{\mathrm{b}}, \mathrm{p}_{\varphi}\right)
$$

imply that the conjugate angles increase linearly in time, even though the regular angles $\theta$ and $\varphi$ do not increase at a constant rate

This linear increase of the canonical angles with time implies that the trajectory is ergodic $^{20}$ on the invariant surface whenever the frequency ratio, $\omega_{b} / \omega_{\zeta}$, is irrational. This implies that a particle samples all angles equally over the course of time. Moreover, the distribution of phases $\zeta$ at constant $\theta_{b}$ is uniform. Finally, it should be mentioned that in the limit of infinite time, a distribution with a small spread of one of the actions phase mixes to where the distribution is uniform in the angles.

When separatrix crossing is present, but the associated small phase-dependent change of the adiabatic invariant is neglected, the situation is the same. There can be a small change of the phase due to separatrix crossing. Nevertheless, as long as the total 
phase change accumulated by making a full poloidal circuit through either of the minority states is irrational, ergodicity on the patched torus follows. Hence, the ensemble for defining the trapping probability is one that is constant in $\theta_{j}$ and uniform in $\zeta_{j}$, when the $j^{\text {th }}$ state is the majority state.

To make this more concrete, we consider the case where the majority state is the positive-passing state. Then for a given value of $\mathrm{J}_{+}$and a given initial launch angle $\theta_{+}, \mathrm{a}$ range $\Delta \zeta_{+r}$ of initial angles $\zeta_{+}$become locally trapped. Since the ensemble is uniform in $\zeta_{+}$, the trapping probability is just $\mathrm{P}_{T}=\Delta \zeta_{+} / 2 \pi$, the trapping range divided by the total range. As the particle passes through the majority state at adiabatic poloidal angle $\theta_{+}$once during each orbit, and the particle has the angle $\zeta_{+}$in the range $\Delta \zeta_{+r}$ the fraction $P_{T}=\Delta \zeta_{+r} / 2 \pi$ of the time, the trapping probability is also the fraction of separatrix encounters for which a particle becomes trapped. A measurement based on this would require a knowledge of the adiabatic angle to calculate the range and a knowledge of the adiabatic invariant to initialize the trajectories at constant J.

To obtain a numerically convenient definition, we note that the transition probability is related to the fluxes of particles converting to the various states. For uniform density in phase space, the flux of particles crossing a constant $-\theta_{+}$surface with adiabatic argle $\zeta_{+}$in the range $\Delta \zeta_{+r}$ adiabatic flux-variable $\psi_{+}$in the narrow range $\delta \psi_{+}$, and adiabatic invariant $J_{+}$in the narrow range $\delta J_{+}$is given by

$$
F_{r}=\iint_{+r} d \zeta_{+r} \iint_{\delta J_{+}} d J_{+} \int \psi_{+} d \psi_{+} \dot{\theta}_{+}=\dot{\theta}_{+} \Delta \zeta_{+r} \delta \psi_{+} \delta J_{+} \cdot
$$

There is no volume factor, since the Jacobian for a canonical transformation is unity. The integration over the adiabatic angle $\zeta_{+}$is trivial, because the rate of increase of the adiabatic angle $\dot{\theta}_{+}$does not depend on the $\zeta_{+}$. The integration over the variables $J_{+}$and $\psi_{+}$follow 
because the range of integration is small. The total upward flux is given be a similar expression, except that the range of the angle $\zeta_{+}$is $2 \pi$. Thus, the relative upward flux is $\Delta \zeta_{+r} / 2 \pi$, which is the trapping probability. In terms of a range $\delta \mathrm{E}$ of energy rather than a range of adiabatic flux-variable the trapping flux is given by

$$
\mathrm{F}_{\mathrm{r}}=\dot{\theta}_{+} \Delta \zeta_{+r} \delta \mathrm{E} \delta \mathrm{J}_{+} / \frac{\partial \mathrm{H}_{+}}{\partial \psi_{+}}=\Delta \zeta_{+r} \delta \mathrm{E} \delta \mathrm{J}_{+} .
$$

Again, the relative flux is the trapping ratio.

Because Hamiltonian flows preserve flux, this holds on an arbitrary surface. This fact provides a second, more convenient method for calculating the trapping probability. By numerical integration one calculates the relative flux of trapping trajectories crossing an arbitrary surface between contours of constant energy and contours of constant adiabatic invariant. (The fact that phase space is four dimensional makes the visualization of the appropriate volume difficult.)

This discussion shows that the transition probability cannot be considered in isolation. Defining the transition probability requires understanding of the averaged dynamics, since the transition probability is defined in terms of the adiabatic angles and invariants. In addition, having the transition probability without the adiabatic description of the dynamics would be pointless, since then one would still have to integrate the guiding-center equations between transitions. 
III.

Adiabatic theory

In the previous section we noted that the trapping probability is given by the ratio of trapping flux to total flux for uniform phase-space density between surfaces of constant adiabatic invariant. Thus, in order to measure the trapping probability numerically, we need to be able to fix the value of the adiabatic invariant. But adiabatic calculations are always carried to finite order, so the question arises as to what order we need to calculate these quantities to have a valid measurement. In the first part of this section we point out that one need know the adiabatic invariant through first order. (This was first noted in Ref. , where the change of the phase between separatrix crossings was first calculated for Hamiltonian systems of one and a half degrees of freedom and slow time dependence.) In the second part of this section we briefly review lowest-order adiabatic theory. Finally, we calculate the first correction to the adiabatic invariant in general. This calculation applies to any Hamiltonian system in which there is a frequency disparity between the motion of two canonical pairs.

\section{A. Required accuracies for measurement of the trapping probability}

One method of numerically determining the trapping probability involves initiating a set of trajectories uniformly on a surface of constant adiabatic angle $\boldsymbol{\theta}_{\mathrm{j}}$ with energy and adiabatic invariant in a small range. However, in practice one has the adiabatic invariant only through some order. So one must initiate the particles at either constant lowest-order or constant first-order adiabatic invariant, etc. By examination of the errors we will determine the order of accuracy needed for the adiabatic invariant and the adiabatic angle. To be specific, we will assume that the majority state is the positive pasing state.

We denote the lowest-order (positive passing) adiabatic invariant by $\mathrm{J}_{+} 0$, the firstorder correction by $\mathrm{J}_{+1}$, and the first-order corrected invariant by $\mathrm{J}_{+}^{1} \equiv \mathrm{J}_{+0}+\varepsilon_{\mathrm{a}} \mathrm{J}_{+1}$. We use $\mathrm{J}_{+}$to refer somewhat imprecisely to the invariant corrected to all orders. [In fact, there 
exists only an asymptotic (probably not convergent) power series for $\mathrm{J}_{+}$. Thus, J actually refers to the power series truncated at some very high order.) We use $\varepsilon_{\mathrm{a}}$ to denote the adiabaticity parameter, the ratio of the time scale of the local motion to that of the poloidal motion, which is also the typical relative size of a correction. In a time $T$ the adiabatic phase of each trajectory increases by

$$
\zeta_{+}(T) \cdot \zeta_{+}(0)=\omega_{\zeta_{+}}\left(J_{+}, E\right) T\left[1+O\left(\varepsilon_{a}^{M}\right)\right]
$$

where $\mathrm{J}_{+}$is the launch value of the true adiabatic invariant, and $\mathrm{M}$ is the (arbitrarily large) order of accuracy of the invariant. The typical time for the drift from one transition point to another is given by $T_{\theta}=O\left(1 / \omega_{\theta_{+}}\left(J_{+}, E\right)\right)$. Hence, the rapid phase increases by a large amount that is $O\left(\omega_{\zeta_{+}}\left(J_{+}, E\right) / \omega_{\theta_{+}}\left(J_{+}, E\right)\right)=O^{\prime}\left(1 / \varepsilon_{a}\right)$ for an order-unity change of the slow phase, as expected. Moreover, the increase is uniform over the ensemble with an error that is at most $O\left(\varepsilon_{a}^{M}\right)$.

Suppose instead the ensemble is one of fixed zeroth order adiabatic invariant, $J_{+0}$. The true adiabatic invariant differs from $\mathrm{J}_{+0}$ by at least $\mathrm{J}_{+}^{1}-\mathrm{J}_{+0}=\varepsilon_{\mathrm{a}} \mathrm{J}_{+}$, and since $\mathrm{J}_{+1}$ is phase dependent, the adiabatic frequency $\omega_{\zeta+}\left(J_{+}, E\right) \approx \omega_{\zeta+}\left(J_{+0}, E\right)+\varepsilon_{a}\left(\partial \omega_{\zeta+} / \partial J_{+}\right) J_{+1}$ will vary by an amount that is $O\left(\varepsilon_{\mathrm{a}}\right)$ over the ensemble. Thus, the rapid phase increases by

$$
\begin{aligned}
\zeta_{+}(\mathrm{T}) & -\zeta_{+}(0)=\left[\omega_{\zeta_{+}}\left(\mathrm{J}_{+}, \mathrm{E}\right)+\varepsilon_{\mathrm{a}}\left(\partial \omega_{\zeta_{+}} / \partial \mathrm{J}_{+}\right) \mathrm{J}_{+1}\right] \mathrm{T}_{\theta}\left[1+\mathrm{O}\left(\varepsilon_{\mathrm{a}}^{\mathrm{N}}\right)\right] \\
& =\omega_{\zeta_{+}}\left(\mathrm{J}_{+}, \mathrm{E}\right) \mathrm{T}_{\theta}\left[1+O\left(\varepsilon_{\mathrm{a}}\right)\right],
\end{aligned}
$$

where the errors in the last expression are phase dependent. Since the first two factors in the last expression are $O\left(1 / \varepsilon_{\mathrm{a}}\right)$, the error term is now order-unity. Note that, actually, this term is small in the ripple strength, so can be neglected for tokamaks. This implies that 
ordinary adiabatic motion may cause phase bunching that is $O(1)$, large enough to make the numerical measurement of the transition probability invalid.

To prevent this, the adiabatic invariant must be held constant through first order in the ensemble. This cannot be taken care of by simply starting the trajectories close to the transition point, since adiabatic theory breaks down there. (In Sec. V-C we compare the results obtained holding the adiabatic invariant constant through first order with those obtained holding it constant only through zeroth order.)

We stress that the higher accuracy requirement for the adiabatic invariant is present only in the numerical measurement of the trapping probability. For other analyses only the lowest-order theory is needed. For example, in a Monte Carlo transport code or a neoclassical theory of transport in the low-collision frequency regime, one needs only the lowest-order adiabatic dynamics, the lowest-order transition probability, and the lowestorder transformation from the usual variables to the adiabatic variables. The lowest-order adiabatic dynamics and transition probability give the motion in the adiabatic variables. The lowest-order transformation gives the location in the physical variables. The additional first-order corrections would add only the small oscillations around the average trajectory, and these oscillations are small compared with change of the particles location due to the drift motion.

\section{B. Hamillonian adiabatic theory for two degrees of freedom}

Cary et al ${ }^{10}$ derived the lowest-order adiabatic invariants using the fact that the poloidal motion in one ripple period is small. This arises (1) because the guiding center drift is small in one ripple bounce or traversal, and (2) because even parallel motion causes a small change $\Delta \theta=t \Delta \varphi=2 \pi t / \mathbb{N}$ of poloidal angle in one traversal of a ripple well. Here we briefly review this calculation. 
Because of the time scale separation, the poloidal variables are the slow variables. while the toroidal variables $\varphi$ and $\mathrm{p}_{\varphi}$ are the fast variables. This time-scale separation may be introduced into the phase-space Lagrangian (14) by inserting an adiabatic parameter $\varepsilon_{\mathrm{a}}$ :

$$
\mathrm{L}_{\phi}=(e \psi / c) \dot{\theta} / \varepsilon_{\mathrm{a}}+\mathrm{p}_{\varphi} \dot{\varphi}-\mathrm{h} .
$$

This adiabatic parameter is a formal ordering parameter. It is put there as an aid to collecting terms of similar magnitudes, but it is set to unity at the end of the calculation. This method of introducing the ordering was used by Littlejohn ${ }^{21}$ in his derivation of the Hamiltonian guiding center equations. It happens to be more convenient than other methods for problems of adiabaticity with two or more degrees of freedom.

Independent variation of the phase-space Lagrangian (25) with respect to all four phase-space variables gives the equations,

$$
\begin{aligned}
& \dot{\theta}=\varepsilon_{\mathrm{a}} \frac{\mathrm{c}}{\mathrm{e}} \frac{\partial \mathrm{h}}{\partial \psi^{\prime}} \\
& \dot{\psi}=-\varepsilon_{\mathrm{a}} \frac{\mathrm{c}}{\mathrm{c}} \frac{\partial \mathrm{h}}{\partial \theta^{\prime}} \\
& \dot{\varphi}=\frac{\partial \mathrm{h}}{\partial \mathrm{p}_{\varphi}}
\end{aligned}
$$

and

$$
\dot{p}_{\varphi}=-\frac{\partial h}{\partial \varphi},
$$

which manifest the slowness of the poloidal motion. These equations are almost of the form of Hamilton's equations, except for the constant factors $c \varepsilon_{\mathrm{a}} / \mathrm{e}$ on the right sides of Eqs. (26a) and (26b). 
The goal of adiabatic theory is to find a transformation 10 new variables $\left(\psi_{\mathrm{j}}, \theta_{\mathrm{j}}, \mathrm{J}_{\mathrm{j}}, \zeta_{\mathrm{j}}\right)$ such that one of them $\left(\zeta_{\mathrm{j}}\right.$, the adiabatic phase for the rapid degree of freedom) is absent from the Hamiltonian. The resulting equations have the simple form of Eqs. (18) and (19). In Ref. 10 the adiabatic invariant is found to be

$$
\left.J_{j}=\frac{1}{2 \pi} \oint d \varphi \rho_{\varphi}(\Psi, \Theta, \varphi, E)\right)
$$

in which

$$
E=h\left(\psi, \theta, p_{\varphi}, \varphi\right)
$$

is the value of the Hamiltonian and $p_{\varphi}(\psi, \theta, \varphi, E)$ is the function obtained by solving Eq. (28) for $p_{\varphi^{*}}$ That is,

$$
p_{\varphi}\left(\psi, \theta, h\left(\psi, \theta, \varphi, p_{\varphi}\right), \varphi\right)=p_{\varphi}
$$

The integration contour of Eq. (27) depends upon the state being considered. For locally trapped particles the contour is the closed contour of $\mathrm{H}$. For passing particles, the contour is actually over one toroidal period at fixed poloidal location. Such a contour may be considered closed since the system is exactly periodic in the toroidal angle.

Inverting Eq. (27) gives the lowest-order adiabatic Hamiltonian $\mathrm{H}_{\mathrm{j} 0}$. Because $\mathrm{H}_{\mathrm{j} 0}$ is independent of the angle $\zeta$, the quantities $\mathrm{J}_{\mathrm{j}}$ are the adiabatic invariants to lowest order.

The evaluation of these adiabatic invariants was carried out in Ref. 10 for a more general model. (In that reference the adiabatic invariant was defined not to have the factor of $2 \pi$.) Here we give the results for the specific model of Eqs. (1) and (2) with the phasespace Lagrangian (14). For locally trapped particles the lowest-order adiabatic invariant is

$$
J_{r_{0}}=\left(4 B_{\varphi} / \pi N\right)\left(\mu m / \varepsilon_{1} B_{0}\right)^{1 / 2} g(\sigma, \eta),
$$


while for passing particles the lowest-order invariant is

$$
J_{ \pm 0}= \pm e A_{\varphi} / N c+\left(2 B_{\varphi} / \pi N\right)\left(\mu m / \varepsilon_{r} B_{0}\right)^{1 / 2} g(\sigma, \eta)
$$

The function $g(\sigma, \eta)$ in these equations is given by

$$
g(\sigma, \eta) \equiv \begin{cases}{[(1+\sigma \eta) \Pi(\sigma, 1 / \eta)-K(1 / \eta)] / \eta^{1 / 2}} & \text { for } \eta>1 \\ (1+\sigma \eta) \Pi(\sigma \eta, \eta)-K(\eta) & \text { for } \eta<1 \\ \sigma^{1 / 2} \arctan \left(\sigma^{1 / 2}\right) & \text { for } \eta=1\end{cases}
$$

in terms of the complete elliptic integral,

$$
K(x) \equiv \int_{0}^{\pi / 2} \frac{d z}{\left(1-x \sin ^{2} z\right)^{1 / 2}}
$$

of the first kind and the complete elliptic integral,

$$
\Pi(y, x) \equiv \int_{0}^{\pi / 2} \frac{d z}{\left(1-x \sin ^{2} z\right)^{1 / 2}\left(1+y \sin ^{2} z\right)}
$$

of the third kind.

To complete the formulas for the adiabatic invariant, we must state what the parameters $\sigma$ and $\eta$ are. The parameter $\eta$ is a measure of the energy of the particle relative to the maximum $\mathrm{V}_{\mathrm{tm}}$ of the potential at fixed poloidal location:

$$
\eta \equiv\left(E-\mu B_{0}\left[1-\varepsilon_{l} \cos (\theta)\right]-e \Phi+\varepsilon_{l} \mu B_{0}\right\} / 2 \varepsilon_{l} \mu B_{0}
$$

This parameter is greater than unity [the first case of Eq. (32)] for passing trajectories, and it is less than unity for trapped trajectories. The other parameter, 


$$
\sigma \equiv 2 \varepsilon_{\mathrm{r}}\left[\left[1-\varepsilon_{\mathrm{l}} \cos (\theta)-\varepsilon_{\mathrm{r}}\right]\right.
$$

is the local relative ripple strength.

\section{First-order corrected adiabatic invariant}

We have noted that the first-order corrections to the adiabatic invariant are needed for the numerical checks. The procedure we use is to write the adiabatic invariant as a series,

$$
J=g_{0}(\psi, \theta, E)+\varepsilon_{\mathrm{a}} d_{1}(\psi, \theta, E, \varphi)+\varepsilon_{\mathrm{a}}^{2} g_{2}(\psi, \theta, E, \varphi)+\ldots
$$

in the criginal variables, and then to determine each term by requiring the adiabatic invariant to be constant to all orders. Our convention is to use the script letters to denote functions of E rather than $P_{\varphi}$.

Through second order the total time derivative of the adiabatic invariant is

$$
\begin{aligned}
j=\varepsilon_{\mathrm{a}} & \left(\frac{\mathrm{c}}{\mathrm{e}} \frac{\partial g_{l}}{\partial \theta} \frac{\partial \mathrm{h}}{\partial \psi}-\frac{\mathrm{c}}{\mathrm{e}} \frac{\partial g_{l}}{\partial \psi} \frac{\partial \mathrm{h}}{\partial \theta}+\frac{\partial g_{1}}{\partial \varphi} \frac{\partial \mathrm{h}}{\partial p_{\varphi}}\right) \\
& +\varepsilon_{\mathrm{a}}^{2}\left(\frac{\mathrm{c}}{\mathrm{e}} \frac{\partial g_{1}}{\partial \theta} \frac{\partial \mathrm{h}}{\partial \psi}-\frac{\mathrm{c}}{\mathrm{e}} \frac{\partial g_{1}}{\partial \psi} \frac{\partial \mathrm{h}}{\partial \theta}+\frac{\partial g_{2}}{\partial \varphi} \frac{\partial \mathrm{h}}{\partial \mathrm{p}_{\varphi}}\right)+\ldots
\end{aligned}
$$

Requiring the first term to vanish determines the first-order correction of the adiabatic invariant up to an initial value:

$$
g_{1}(\varphi)=g_{1}\left(\varphi_{0}\right)+\frac{c}{e} \int_{\varphi_{0}}^{\varphi} d \varphi^{\prime} \frac{\partial p_{\varphi}}{\partial E}\left[h, g_{0}\right]
$$

Here we have introduced the poloidal Poisson Bracket, 


$$
\left[\mathrm{h}, g_{0}\right] \equiv \frac{\partial \mathrm{h}}{\partial \theta} \frac{\partial g_{0}}{\partial \psi}-\frac{\partial \mathrm{h}}{\partial \psi} \frac{\partial g_{0}}{\partial \theta}
$$

and the function $p_{\varphi}(\psi, \theta, E, \varphi)$ of Eq. (29).

Differentiating Eq. (29) with respect to $\psi$ and $\theta$ gives the relation,

$$
\frac{\partial p_{Q}}{\partial E} \frac{\partial h}{\partial \psi}=-\frac{\partial p_{Q}}{\partial \psi}
$$

and a similar relation with $\psi$ replaced by $\theta$. Use of these relations puts Eq. (38) in the simplified form,

$$
g_{1}(\varphi)=g_{1}\left(\varphi_{0}\right)+\frac{c}{e} \int_{\varphi_{0}}^{\varphi} d \varphi^{\prime}\left[\delta_{0}, p_{\varphi}\right] .
$$

An identical procedure give the second correction,

$$
g_{2}(\varphi)=g_{2}\left(\varphi_{0}\right)+\frac{c}{e} \int_{\varphi_{0}}^{\varphi} d \varphi^{\prime}\left[g_{1}, p_{\varphi}\right] .
$$

In previous work ${ }^{16}$ a similar analysis was carried out for the case of a Hamiltonian with slow time dependence. There it was shown that the initial value in Eq. (41) is determined by a solvability condition. The solvability condition is the condition that the corrections to the adiabatic invariant be single valued in phase space. Thus, one must have $g_{n}\left(\varphi_{0}+2 \pi\right)+g_{n}\left(\varphi_{0}\right)$ for passing particles, while for trapped particles the value of $g_{n}\left(\varphi_{0}\right)$ must not change after adding the integral around the phase-space loop in Eqs. (41) and (42). In Ref. 10 it was shown that this condition is satisfied for $\mathscr{g}_{1}$ when $g_{0}$ is the lowestorder adiabatic invariant. Invoking this condition for $g_{2}$ gives the condition, 


$$
0=\oint d \varphi\left[g_{1}, p_{\varphi}\right]=\oint d \varphi\left[g_{1}\left(\varphi_{0}\right), p_{\varphi}\right]+\frac{c}{e} \oint d \varphi\left[\int_{\varphi_{0}}^{\varphi} d \varphi^{\prime}\left[g_{0}, p_{\varphi}^{\prime}\right], p_{\varphi}\right],
$$

for the initial condition $g_{1}\left(\varphi_{0}\right)$. Here we have introduced the shorthand, $P_{\varphi}^{\prime} \equiv p_{\varphi}\left(\varphi^{\prime}\right)$. It is understood that the loop integral is over one period for passing particles and around a phase-space loop for trapped particles.

The first term on the right of Eq. (43) is $\left[g_{1}\left(\varphi_{0}\right), g_{0}\right]$. The remaining term is simplified through use of the Jacobi identity and integration by parts. Applying the Jacobi identity to half of this term gives

$$
\begin{aligned}
\frac{c}{\mathrm{e}} \oint \mathrm{d} \varphi\left[\int_{\varphi_{0}}^{\varphi} \mathrm{d} \varphi^{\prime}\left[\delta_{0}, p_{\varphi}^{\prime}\right], p_{\varphi}\right]=-\frac{c}{2 \mathrm{e}}\left[\oint d \varphi \int_{\varphi_{0}}^{\varphi} \mathrm{d} \varphi^{\prime}\left[p_{\varphi^{\prime}}^{\prime}, p_{\varphi}\right], \delta_{0}\right] \\
+\frac{c}{2 \mathrm{e}} \oint d \varphi \int_{\varphi_{0}}^{\varphi} \mathrm{d} \varphi^{\prime}\left(\left[\left[\delta_{0}, p_{\varphi}^{\prime}\right], p_{\varphi}\right]+\left[\left[\delta_{0}, p_{\varphi}\right], p_{\varphi}^{\prime}\right]\right) .
\end{aligned}
$$

Integration by parts shows that the last term in this equation vanishes. This result, inserted into Eq. (43), implies that

$$
\left[A, g_{0}\right]=0 \text {, }
$$

where

$$
A \equiv g_{1}\left(\varphi_{0}\right)-\frac{c}{2 c} \oint d \varphi \int_{\varphi_{0}}^{\varphi} d \varphi^{\prime}\left[p_{\varphi}^{\prime}, p_{\varphi}\right]
$$

must be satisfied by the initial condition.

The condition (44a) is satisfied by letting $A$ be any function of the lowest order invariant, $8_{0}$. The simplest choice is to set A to zero. As noted in Ref. 16, this is 
equivalent to imposing the additional condition that a contour of the corrected invariant, $g_{0}+\varepsilon_{\mathrm{a}} g_{1}$, enclose phase space area equal to the value of the invariant on that contour, a condition satisfied by the lowest-order invariant. With the choice, $A=0$, Eq. (44b) gives the first order correction and any value of the toroidal angle, since the initial point is arbitrary. Thus,

$$
\begin{aligned}
g_{1}(\varphi) & =\frac{c}{4 \pi \mathrm{e}} \oint \mathrm{d} \varphi^{\prime} \int_{\varphi}^{\varphi^{\prime}} \mathrm{d} \varphi^{\prime \prime}\left[p_{\varphi}\left(\varphi^{\prime \prime}\right), p_{\varphi}\left(\varphi^{\prime}\right)\right] \\
& =-\frac{\mathrm{c}}{2 \mathrm{c}} \frac{\partial \partial_{0}}{\partial \theta} \frac{\partial \delta_{0}}{\partial \psi}+\frac{\mathrm{c}}{\mathrm{e}} \oint \mathrm{d} \varphi^{\prime} \frac{\partial p_{\varphi}}{\partial \psi}\left(\varphi^{\prime}\right) \int_{\varphi^{\prime}}^{\varphi^{\prime}} \mathrm{d} \varphi^{\prime \prime} \frac{\partial p_{\varphi}}{\partial \theta}\left(\varphi^{\prime \prime}\right) .
\end{aligned}
$$

This general formula for the correction may now be evaluated for any of the particular cases of interest. This correction is evaluated for the standard model in App. B. 
IV. Calculation of the trapping probability

In Sec. II we noted that adiabatic theory predicts that particles will stay approximately on invariant surfaces until they encounter a transition curve, where they must change state. For example, a negative-passing particle at its banana tip must either reflect to become positive passing, or it must become locally trapped. In the last section we reviewed the theory of the adiabatic invariants and showed how to calculate them through first order. In the present section we show how the transition probability, which determines the fraction of particles that enter a particular state, can be calculated. Our calculation is based on the fact that the probability of becoming trapped is simply the relative flux of trajectories that become trapped, as was shown in Sec. II. Conservation of flux implies that this argument holds even near the transition point, where adiabatic theory breaks down. Our calculation is basically of two parts. The first part is to determine the regions of phase space which become trapped or reflected. The second is to calculate the relative fluxes of the two processes in this region.

\section{A. Trapping region on a given field line}

If drifts are neglected, particles stay on field lines. In this approximation, no trapping takes place. Energy conservation ensures that a particle moving towards smaller major radius with sufficient energy to cross a local maximum of the potential energy will still have sufficient energy upon its retum. However, drifts cause particle to move to a different field lines on which a local maximum may be larger, so that the particle becomes trapped.

Figure 4 indicates the trapping and reflection processes in an energy diagram. This heavy curve is the potential energy along a field line. The trajectory labeled $c$ asymptotes to the top of the left local maximum, the trajectory labeled $a$ asymptotes to the right local maximum, while the trajectory labeled $b$ asymptotes to the left local maximum after 
reflecting off the right maximum. This diagram is not precise because energy is in fact conserved. The diagram indication of decreasing energy for these particles is actually a representation of the fact that the drift to neighboring field lines is causing the potential energy on a field line to increase as shown in Fig. 2. Here and below, we use the term potential energy in the generalized sense of $\mu \mathrm{B}+\mathrm{e} \Phi$.

Figure 5 is a representation of this same process in phase space. The heavy curves in this diagram are contours of the Hamiltonian along a field line, i.e., at fixed $\beta$. Were there no drifts, a trajectory would move along one of these lines, since energy is conserved. But the drifts cause the local potential-energy wells to increase in size. As a result trajectories are a drawn into these wells and become trapped. As before, this is represented somewhat misleadingly by showing the trajectory $b$ spiraling into the well, because we cannot show the well increasing in size.

The present goal is to determine the class of trajectories starting on a particular field line that become trapped between two of the maxima in Fig. 4. We do this by determining the ranges of the energy relative to that of the local maximum for the various classes of particles. Here we improve on the results Ref. 10 by including poloidal motion due to rotational transform in lowest order.

To include the rotational transform we will make the approximation that $\beta$ is nearly constant rather than $\theta$. Differentiating Eq. (4a) and inserting the result into the phase-space Lagrangian (14) gives

$$
L_{\phi}=\frac{e}{c} \psi \dot{\beta}+\operatorname{mub}_{\varphi} \dot{\varphi}-h
$$


plus a total time derivative, that of $\left(A_{\varphi}++\psi\right) \varphi$, which has no effect on the equations of motion and so may be discarded from the Lagrangian. (We have now set the formal ordering parameter $\varepsilon_{\mathrm{a}}$ to unity.) Thus, in these coordinates the conjugate momentum to $\varphi$ is

$$
v_{\varphi} \equiv \operatorname{mub}_{\varphi}
$$

The vector potential does not appear. Similarly, the momentum conjugate to $\beta$ is now $\psi$. Thus, in this subsection we will use these as our coordinates, so that, for example, $\partial h / \partial \psi$ is the derivative at constant $\beta, v_{\varphi}$, and $\varphi$.

The trajectory $b$ is one for which the value of $E-E_{0}$ has decreased to zero after completing one ripple well circuit, i.e., reflecting off the maximum at $\varphi_{1}$ and returning to $\varphi_{0}$, where $E_{0}$ is the value of the energy on the local potential maximum at $\varphi_{0}$. Since $E$ is conserved in the flow, this implies that $E_{0}$ has increased in one circuit by the amount $\Delta E_{r}$. defined to be the value of $E-E_{0}$ for trajectory $b$ as it first crosses the maximum at $\varphi_{0}$. The change of $E_{0}$ is given by the integral of its total time derivative,

$$
\begin{aligned}
\Delta E_{0} & =\int d q\left(\frac{\partial E_{0}}{\partial \psi} \frac{d \psi}{d t}+\frac{\partial E_{0}}{\partial \beta} \frac{d \beta}{d t}\right) \\
& \approx \frac{\partial E_{0}}{\partial \psi} \int d \varphi \frac{d \psi}{d t} / \frac{d \varphi}{d t}+\frac{\partial E_{0}}{\partial \beta} \int d \varphi \frac{d \beta}{d t} / \frac{d \varphi}{d t} \\
& =-\frac{\partial E_{0}}{\partial \psi} \int d \varphi \frac{m c}{e} \frac{\partial h}{\partial \beta} / \frac{\partial h}{\partial v_{\varphi}}+\frac{\partial E_{0}}{\partial \beta} \int d \varphi \frac{m c}{e} \frac{\partial h}{\partial \psi} / \frac{\partial h}{\partial v_{\varphi}} .
\end{aligned}
$$

The last expression comes from the approximation that $E_{0}$ varies little in one ripple period of the motion, so that the derivatives of $\mathrm{E}_{0}$ can be taken out of the integrand and evaluated at some point along the trajectory. We have now dropped the formal ordering parameter $\varepsilon_{\mathrm{a}}$. 
The integrands in Eq. (48) can be rewritten as derivaluves of the function $v_{\varphi}(\psi, \beta, E, \varphi)$ which is analogous to $p_{\varphi}$ of Eq. (29). That is, $v_{\varphi}$

$$
v_{\varphi}\left(\psi, \beta, h\left(\psi, 1 \quad, v_{\varphi}\right), \varphi\right)=v_{\varphi} .
$$

is found by solving for $v_{\varphi}$ as a function of the energy at constant values of $\psi$ and $\beta$. Differentiation of Eq. (49) with respect to $\psi$ and $\beta$ yields formulas that permit Eq. (48) to be simplified to

$$
\Delta \mathrm{E}_{0}=\frac{\mathrm{c}}{\mathrm{e}}\left(\frac{\partial \mathrm{E}_{0}}{\partial \psi} \int \mathrm{d} \varphi \frac{\partial \mathrm{v}_{\varphi}}{\partial \beta}-\frac{\partial \mathrm{E}_{0}}{\partial \beta} \int \mathrm{d} \varphi \frac{\mathrm{d} v_{\varphi}}{\partial \psi}\right)
$$

The last approximation we make is to replace the integrals in Eq. (50), which are along the true trajectory, by integrals along the separatrix, the constant-energy curve labeled 0 in Fig. 5. The justification for this replacement is that the drift is small in one orbit, so the trajectories near the separatrix can be replaced by the trajectory on the separatrix. After making this replacement, the partial derivatives may be taken outside of the integrals to obtain

$$
\Delta E_{0}=\frac{c}{e}\left[Y_{0}, E_{0}\right]_{\beta} \text {, }
$$

where

$$
Y_{0} \equiv \oint_{E=E_{0}} v_{\varphi} d \varphi
$$

is the loop integral on the separatrix (the contour $E=E_{0}$ ), and the subscript on the bracket in Eq. (51) indicates that Eq. (49) is to be used with $\theta$ replaced by $\beta$. This formula shows that the change of $\mathrm{E}_{0}$ depends on only the poloidal location of the crossing of the last 
maximum. It is independent of the initial value of $E-E_{0}$, provided this is small, so that the integration along the trajectory can be replaced by the integration along the separatrix.

This calculation of the change of $E_{0}$ is valid provided the trajectory finishes its circuit of the separatrix before drifting too far. For the particular trajectory $b$ that asymptotes into the maximum, this analysis breaks down, because this trajectory drifts far away in $\psi$ and $\theta$ from where it first crossed this maximum, and so the approximations involved in taking the derivatives of $\mathrm{E}_{0}$ outside the integral are bad. However, as has been shown in Ref. 17, for only an exponentially small (in the adiabaticity parameter) relative number of trajectories does this approximation fail. For trajectories slightly above or slightly below $b$ the change of $\mathrm{E}_{0}$ is given by Eq. (51). This implies that the difference between the energy of orbit-b and that of orbit-c is close to $-\Delta\left(E-E_{0}\right)$ to within an exponentially small factor:

$$
\Delta E_{\mathrm{r}}=\frac{\mathrm{c}}{\mathrm{e}}\left[\mathrm{Y}_{0}, \mathrm{E}_{0}\right]_{\beta}
$$

An analogous argument give the difference $\Delta \mathrm{E}_{+}$in energy between trajectory $a$ and the energy $E_{1}$ of the right maximum:

$$
\Delta E_{+}=\frac{1}{2} \frac{c}{e}\left[Y_{1}, E_{1}\right]_{\beta}
$$

where

$$
Y_{1}=2 \int_{\varphi_{0}}^{\varphi_{1}} d \varphi v_{\varphi}
$$

is defined in parallel to be the difference between the phase-space area enclosed by the both halves of the contour $\mathrm{E}=\mathrm{E}_{1}$ (the value of the energy at the next maximum on this field line) 
in one ripple period and the sepatarix corresponding to the right maximum. However, only half this integral is kept in Eq. (54), since the trajectory $c$ only executes the positivevelocity portion before encountering the local maximum.

The total energy range of trapping or reflected trajectories is

$$
\Delta \mathrm{E}_{\mathrm{T}}=\Delta \mathrm{E}_{\mathrm{x}}+\Delta \mathrm{E}_{+} \text {, }
$$

where

$$
\Delta E_{x}=E_{1}-E_{0}
$$

is the difference in energy between the two maxima on a field line. Thus, we conclude that on a given field line the trajectories that trap or reflect between the 0-maximum and the 1-maximum are those with $0<E-E_{0}<\Delta E_{T}$, while the trajectories that trap are those with

$0<\mathrm{E}-\mathrm{E}_{0}<\Delta \mathrm{E}_{\mathrm{r}}$. We must now calculate the fluxes in these regions in order to determine the trapping probability.

\section{B. Flux across the local maximum}

We recall from Sec. II that the transition process can be considered as follows. An ensemble of trajectories uniform in phase space with energy and corrected adiabatic invariant in some small fixed ranges and $\varphi$ throughout a field period is initiated at some surface. These trajectories move along magnetic field lines and drift to other surfaces, eventually coming to a poloidal position where the trajectories must either reflect or trap. The relative flux of trajectories that trap is the trapping probability.

This transition process is shown in Fig. 6, where an incoming positive passing particle is either reflected or trapped depending on its energy relative to the local maxima (on a field line) of the potential encountered by the trajectory. The solution, 


$$
\chi=2 \pi n \cdot \arcsin (\alpha)
$$

of Eq. (5) for the location of these maxima, shows that these maxima are lines of constant $\chi$ when $\varepsilon_{\mathrm{t}}+\mathbb{N} \varepsilon_{\mathrm{r}}$ is small. These are the dashed lines of Fig. 6.

The field line (constant $\beta$ ) passing through point $c$ separates two types of trajectories. The trajectory that comes in just above this field line see a higher value of potential maximum, so it is reflected by the potential maximum at $\chi$ near zero. The trajectory that comes in below this field line sees a lower value of the maximum, and since it has the same energy, is able to pass over the local maximum. It, however, is reflected by the next maximum at $\chi$ near $2 \pi$. Its drifting motion continues to increase the value of the local maximum. By the time the trajectory has retumed to the local maximum near $\chi=0$, it has drifted to a field line on which the energy of the trajectory is less than that of the local maximum. Hence, the particle is reflected again and becomes trapped in the local magnetic well. The field line passing through point $c^{\prime}$ is exactly one period to the right of the field line passing through pont $c$, and so the dynamics on this line is the same. Thus, all of the trajectories cross the maximum line near zero between points a (the projection backward of c) and $c$.

Trajectories crossing this line farther below point $c$ cross the local maximum with greater energy relative to the maximum. A trajectory crossing above point $b$ is just barely reflected by the first local maximum on its retum from the second local maximum. A trajectory crossing just below this point does have sufficient energy to cross back over the local maximum and, hence, does not become trapped. In summary, trajectories which cross the local maximum between points $b$ and $c$ become trapped, while those that cross between points $a$ and $b$ reflect, becoming negative passing. To calculate the trapping probability we need to calculate the relative fluxes crossing the surface of constant $\chi$ 
between these points for energy and inital adiabatic invariant in some infinitesinal ranges and initial $\varphi$ throughout a period.

The flux crossing a surface of constant $\chi$ is easily described in yet another variable set in which one of the variables is $\chi$. Replacing the variable $\varphi$ by the variable $\chi$ in the phase-space Lagrangian (14) yields

$$
L_{\phi}=p_{\theta} \dot{\theta}+p_{\chi} \dot{\chi} \cdot h,
$$

where

$$
p_{\chi} \equiv p_{\Phi} N
$$

and

$$
p_{\theta} \equiv e \psi / c+l p_{\varphi} N=(e / c)\left(\psi+l A_{\varphi} N\right)+l m u b_{\varphi} N
$$

Thus, the variables $\left(\theta, p_{\theta}\right)$ and $\left(\chi, p_{\chi}\right)$ form canonical pairs with the equations of motion being Hamilton's equations.

In these variables the differential flux crossing a surface of constant $\chi$ is given by

$$
d F=\dot{\chi} d p_{\chi} d \theta d p_{\theta}
$$

The volume element is unity because these variables are canonical. This also implies that Hamilton's equation, $\dot{\chi}=\partial \mathrm{h} / \partial \mathrm{p}_{\chi}$ (at constant $\theta$ and $\mathrm{p}_{\theta}$ ) gives the first factor. Hence, the differential flux is given by

$$
d F=\frac{\partial h}{\partial p_{\chi}} d p_{\chi} d \theta d p_{\theta}=d E d \theta d p_{\theta}
$$


So, the flux equals the integral of the element (62) over the surface of constant $\chi$ over the region corresponding to the initial ensemble of energy within a differential range $\delta E$, corrected adiabatic invariant within a differential range $\delta \mathrm{J}$, and $\varphi$ throughout a period.

We note that all trajectories cross the maximum at $\varphi_{0}$ within a small range of $\psi$ and $\theta$ values. To determine the range of $\theta$ near the transition region, we refer to Fig. 6 . We note that points $c$ and $c^{\prime}$ have the same value of $\theta$ but are separated by $\Delta \varphi=2 \pi / N$ and, hence, $\Delta \beta=2 \pi / q N$. Thus, points $a$ and $c$ differ by $\Delta \beta=2 \pi / q N$, since points $a$ and $c^{\prime}$ are on the same field line. Finally, points $a$ and $c$ have approximately the same value of $\chi$. Setting $\Delta \chi=N q \Delta \beta+(N q-\sqcap) \Delta \theta=0$, gives the angular separation of

$$
\Delta \theta=2 \pi /(\mathrm{Nq}-l)
$$

between points $a$ and $c$, which is small when the number of field periods is large. In addition, there is a variation of the poloidal location due to the drifts, but this is even smaller for all but an exponentially small class of particles.

The range of energies in the differential flux need not be infinitesimal, but only small enough such that all of the trajectories have essentially the same dynamics. Hence, we broaden the range to be $\Delta \mathrm{E}_{\mathrm{T}}$. This is valid because trajectories in this range of energies all cross the maximum at $\varphi_{0}$ within a range of $\theta$ and $\psi$ that is small, as just noted, and so have approximately the same dynamics.

The total flux of trajectories reflecting or trapping between $\varphi_{0}$ and $\varphi_{1}$ is then given by 


$$
\begin{aligned}
& F_{T}=\int_{\delta J} d p_{\theta} d \theta \int_{\Delta E_{T}} d E=\int_{\delta J ~} \int_{0<\varphi<2 \pi / N} d p_{\theta} d \theta \Delta E_{T} \\
& \approx \Delta E_{T} \int_{\delta J \varphi<2 \pi / N} d p_{\theta} d \theta
\end{aligned}
$$

an integral over the region of the constant- $\chi$ surface corresponding to the initial ensemble. The last approximate equality follows from the fact that the variation of $\psi, \theta$ and, hence. $\Delta \mathrm{E}_{\mathrm{T}}$ over this surface is small, so that $\Delta \mathrm{E}_{\mathrm{T}}$ may be taken outside the integral. The flux of trajectories that become trapped has the identical form, except that $\Delta \mathrm{E}_{\mathrm{T}}$ is replace by $\Delta \mathrm{E}_{\mathrm{r}}$. Thus, we deduce that the trapping probability is given by

$$
P_{r}=F_{r} / F_{T}=\Delta E_{r} / \Delta E_{T}=\left[Y_{0}, E_{0}\right]_{\beta} /\left(\Delta E_{x}+\frac{1}{2}\left[Y_{1}, E_{1}\right]_{\beta}\right)
$$

where $\Delta E_{\mathrm{T}}$ is given by $\mathrm{Eq}$. (53) and $\Delta \mathrm{E}_{\mathrm{T}}$ is given by Eq. (56).

In subsequent sections we will calculate the quantities $E_{j}, Y_{j}$, and $\Delta E_{x}$ as functions of the poloidal angle rather than the field-line variable $\beta$. Thus, it is useful to express the transition probability in terms of the $\theta-\psi$ bracket of Sec. III. These two brackets are related by the derivative $\partial \theta, \partial \beta$ along the maximum:

$$
\left[Y_{1}, E_{1}\right]_{\beta}=\left[Y_{1}, E_{1}\right] \frac{\partial \theta}{\partial \beta}
$$

Solving Eqs. (3), (4), and (58) gives the condition of being at the maximum as

$$
\chi=(\mathrm{Nq}-l) \theta-\mathrm{Nq} \beta=2 \pi \mathrm{n}-\arcsin (\alpha) .
$$

In deriving the trapping probability we previously made the approximation that $\chi$ was nearly constant along the maximum. Hence, in differentiation of the second equality with respect to $\theta$, the variation of $\arcsin (\alpha)$ must be ignored: 


$$
\frac{\partial \beta}{\partial \theta}=1-l t / \mathbb{N}
$$

Hence, our final result for the trapping probability is

$$
P_{r}=\left[Y_{0}, E_{0}\right] /\left\{\Delta E_{x}(1-l t / N)+\frac{1}{2}\left[Y_{1}, E_{1}\right]\right]
$$

The argument we have presented provides better justification of the analysis of Ref. 10 and further improves upon it. In that work the formula (66) was obtained, except that the actions in Eqs. (52) and (55) were integrals along contours of the Hamiltonian at fixed poloidal location, and the factor $(1-1+/ N)$ in the denominator of Eq. (66) was absent. This is in essence a neglect of the lowest order motion along field lines due to finite rotational transform per field period. The present calculation is an improvement in that the integrals are along contours of the Hamiltonian at fixed $\beta$, i.e., on a fixed field line. Hence, the nonzero rotational transform is not treated perturbatively. Only the drifts are treated perturbatively.

The analysis so far presented is limited in that we have assumed that the positive passing state is the majority state. To calculate the trapping probability for cases when the majority state is the negative passing state or the locally trapped state, it is convenient to introduce nomalized incoming fluxes. The trapping probability $(66)$ is the ratio of the flux of trajectories that become trapped to the incoming flux of all of the positive passing trajectories that either trap or reflect. Hence, we define

$$
f_{+} \equiv \Delta E_{x}(1-l+\mathbb{N})+\frac{1}{2}\left[Y_{1}, E_{1}\right]
$$


the be the normalized incoming positive passing flux when $\Delta \mathrm{E}_{\mathrm{x}}$ is greater than zero, i.e. above the midplane in this model. This is not exactly the flux, because the factor $\partial \theta / \partial \beta$ and the integrals seen in Eq. (64) have been divided out.

The normalized incoming flux of ripple trapped trajectories is given by

$$
f_{r} \equiv-\left[Y_{0}, E_{0}\right]
$$

This is the negative of the numerator of the trapping probability (66), because this numerator is the outgoing (i.e., away from the transition surface) normalized flux of trapping trajectories. Because of flux conservation, the outgoing negative passing flux must equal the difference between the incoming positive passing flux and the outgoing trapping flux. The incoming negative passing flux is the negative of the outgoing negative passing flux. Hence, the incoming normalized negative passing flux is

$$
f_{.} \equiv-\Delta E_{x}(1-l z / N)-\frac{1}{2}\left[Y_{1}, E_{1}\right]+\left[Y_{0}, E_{0}\right]
$$

Flux conservation, used to find Eq. (69), dictates that the sum of the incoming fluxes adds to zero.

$$
f_{+}+f_{-}+f_{r}=0
$$

In fact, our calculations of these fluxes did not rest on whether the positive passing state was the majority state, but only on the Figs. 4-6, which assumed that $\Delta E_{x}$ was positive. So we can now simply calculate the quantities $f_{j}$ and from them deduce the majority state and the trapping probability. Since the $f_{j}$ add up to zero, either one of them or two of them are positive. If one is positive, say state $m$, the situation is as we discussed. That state is the majority state, since it is the only state with incoming flux. The probability of making the transition to state $n$, one of the other states, is 


$$
P_{n}=-f_{n} / f_{m} \text {, }
$$

the ratio of the outgoing flux of state $n$ (hence, the minus sign) to the incoming flux of state m. This transition probability is guaranteed to be less than unity, as Eq. (70) dictates that the sum of the magnitudes of the $f_{n}$ for the minority states equals $f_{m}$, and, hence, each of the $f_{n}$ is less that $f_{m}$ in magnitude.

If two of the $f_{j}$ are positive, then trajectories of those two states are incoming, and there is only one outgoing state, which must, therefore, be the majority state. The probability of transition to that outgoing state is unity. Thus, we have shown how to eliminate the problem of having probability greater than unity.

Lastly, we mention the minor changes necessary when $\Delta \mathrm{E}_{\mathrm{x}}$ is negative. In this case, the calculation proceeds as before, except that now the minima labeled 0 and 1 have interchanged as have the roles of the negative passing and positive trajectories. Thus, the incoming flux of negative passing trajectories is

$$
f_{.}=\left|\Delta E_{x}\right|(1-l t / N)+\frac{1}{2}\left[Y_{0}, E_{0}\right]
$$

the incoming flux of trajectories that become trapped is

$$
\mathbf{f}_{\mathbf{r}}=-\left[\mathrm{Y}_{1}, \mathrm{E}_{1}\right]
$$

and the incoming flux of positive passing trajectories is

$$
f_{+}=\left|\Delta E_{x}\right|(1-l t / N)-\frac{1}{2}\left[Y_{0}, E_{0}\right]+\left[Y_{1}, E_{1}\right]
$$

The majority state and transition probability follow as in the previous discussion. 
The standard stellarator model and most others have the property that the ripple averaged motion is up-down symmetric. This symmetry implies the $\Delta E_{()}, \Delta E_{1}, Y_{0}$ and $Y_{1}$ are even in $\theta$. It is this symmetry that guarantees that the three invariant contours which meet at the upper half of the transition contours shown in Fig. 3 meet also at the lower half. A consequence of this symmetry is that the quantities $f_{j}$ are odd in $\theta$, because they are constructed by taking a single $\theta$ derivative of the even quantities. Hence, the analysis is consistent, because the two incoming states at one transition point are outgoing at the other, with incoming flux at one point equaling outgoing flux at the other.

\section{Evaluation of the trapping probability}

The trapping probability has now been expressed through Eqs. (52), (55), (57), and (66) or (71) in terms of the values of the energies on the separatrices and the poloidal derivatives of these energies and the phase-space areas enclosed by the associated contours of the Hamiltonian. The evaluation of these energies in closed form is not possible for the actual potential energy. Hence, we develop a model for the potential energy along a field line.

Our model (see Fig. 7) is based on the expansion of the toroidal term in the magnetic field about the nominal point $\left(\theta_{0}, \varphi_{0}\right)$ transition point of transition that for best accuracy should be midway between the potential maxima. That is, we take

$$
\chi=\pi+\mathrm{x}
$$

and $\theta=\theta_{0}+\delta \theta=\theta_{0}+t \delta \varphi$. Since $x=\delta \chi=N \delta \varphi-1 \delta \theta$ along a field line, we have $\theta=\theta_{0}+x /(\mathrm{Nq}-l)$. Expansion then gives

$$
\mathrm{V}=\mu \mathrm{B}_{0}\left[1-\varepsilon_{\mathrm{t}} \cos \left(\theta_{0}\right)+\varepsilon_{\mathrm{t}} \sin \left(\theta_{0}\right) \mathrm{x} /(\mathrm{Nq}-l)+\varepsilon_{\mathrm{r}} \cos (\mathrm{x})\right]+\varepsilon \Phi(\psi)
$$


Such an expansion (used also by Goldston and Towner $^{7}$ )is a good approximaluon provided $\mathrm{x}$ is not too large - essentially that $2 \pi /\left(\mathrm{Nc}_{4}-l\right)$ be small. In this approximation the potential of Fig. 4 is the sum of a straight line and a sim

nusoidal function. Even in this approximation it is not possible to obtain simple results for the separatrix action, so we examine the potential further with an eye towards additional approximations.

The addition of the sinusoid displaces the maxima and minima from the usual $x=n \pi$. Differentiating with respect to $x$ shows that the extrema satisfy $\sin (x)=-\alpha$, with $\alpha$ evaluated at $\theta_{0}$. Thus, the minimum is located at

$$
x_{m n}=-\arcsin (\alpha)
$$

where the potential energy is

$$
E_{m n}=e \Phi+\mu B_{0}\left[1-\varepsilon_{t} \cos \left(\theta_{0}\right)-\varepsilon_{r}\left(\alpha \arcsin (\alpha)-\sqrt{1-\alpha^{2}}\right)\right]
$$

The left maximum is located at

$$
x_{0}=-\pi+\arcsin (\alpha)
$$

where the potential energy is

$$
E_{0}=e \Phi+\mu B_{0}\left[1-\varepsilon_{t} \cos \left(\theta_{0}\right)+\varepsilon_{r}\left(\alpha[\arcsin (\alpha)-\pi]+\sqrt{1-\alpha^{2}}\right)\right]
$$

The right maximum is located at

$$
x_{1}=\pi-\arcsin (\alpha)
$$

where the potential energy is 


$$
E_{1}=e \Phi+\mu B_{0}\left[1-\varepsilon_{t} \cos \left(\theta_{0}\right)+\varepsilon_{r}\left(\alpha[\arcsin (\alpha)+\pi]+\sqrt{1-\alpha^{2}}\right)\right]
$$

Thus, the energy difference between the successive maxima is given by

$$
\Delta \mathrm{E}_{\mathrm{x}}=2 \pi \mu \mathrm{B}_{0} \varepsilon_{\mathrm{l}} \sin \left(\theta_{0}\right) /(\mathrm{Nq}-l),
$$

the height of the left maximum (Fig. 7) of the magnetic field relative to the minimum is

$$
2 \Delta_{0} \equiv\left[B\left(x_{0}\right) \cdot B\left(x_{m n}\right)\right] / B_{0}=\varepsilon_{r}\left(\alpha[2 \arcsin (\alpha)-\pi]+2 \sqrt{1-\alpha^{2}}\right),
$$

and the height of the right maximum (Fig. 7) of the magnetic field relative to the minimum is

$$
2 \Delta_{1} \equiv\left[B\left(x_{1}\right)-B\left(x_{m n}\right)\right] / B_{0}=\varepsilon_{r}\left(\alpha[2 \arcsin (\alpha)+\pi]+2 \sqrt{1-\alpha^{2}}\right) .
$$

The approximation we now introduce is to make the potential of Fig. 4 out of piecewise sinusoids joined smoothly. That is, we let

$$
B / B_{0}=\left\{\begin{array}{ll}
1-\varepsilon_{t} \cos \left(\theta_{0}\right)-\pi \alpha \varepsilon_{r}-\Delta_{0} \cos \left(k_{0}\left(x-x_{m n}\right)\right) & \text { for } x_{0}<x<x_{m n} \\
1-\varepsilon_{t} \cos \left(\theta_{0}\right)+\pi \alpha \varepsilon_{r}-\Delta_{1} \cos \left(k_{1}\left(x-x_{m n}\right)\right) & \text { for } x_{m n}<x<x_{1}
\end{array}\right. \text {, }
$$

where

$$
k_{j} \equiv \pi / x_{j}-x_{m n}
$$

This is a uniform approximation that is very good even for large values of $\alpha$. This approximation aids in simplification in that all of the separatrix actions are now expressible in terms of complete elliptic integrals. 
We now proceed to evaluate the separatrix actions in this model. To calculate the action $Y_{0}$ of Eq. (52) we express the integral as a sum over each of the regions of Eq. $(81)$. This gives

$$
Y_{0}=\frac{1}{1-l t / N}\left(\frac{2}{k_{0}} \int_{x_{0}}^{x_{m n}} d\left(k_{0} x / N\right) m u B_{\varphi} / B+\frac{2}{k_{1}} \int_{x_{m n}}^{x_{R}} d\left(k_{1} x / N\right) m u B_{\varphi} / B\right)
$$

where $x_{R}$ is the right turning point for the contour of energy $E_{0}$. Each of these integrals has the form of the integral (27) that gives rise to the expression (30), except that each of these integrals would give only half of the action contribution had they the same parameters. The first integral is one half of Eq. (30) evaluated at $\eta=1$ and at

$$
\sigma=\sigma_{0} \equiv 2 \Delta_{0}\left[1-\varepsilon_{\mathrm{t}} \cos \left(\theta_{0}\right)-\pi \alpha \varepsilon_{\mathrm{r}}-\Delta_{0}\right]
$$

Expressions for $9(\sigma, \eta)$ for $\eta$ near unity are given in App. A. The second integral is one half of Eq. (30) evaluated at $\eta=\Delta_{0} / \Delta_{1}$ and

$$
\sigma=\sigma_{1} \equiv 2 \Delta_{1} /\left[1 \cdot \varepsilon_{1} \cos \left(\theta_{0}\right)+\pi \alpha \varepsilon_{\mathrm{r}} \cdot \Delta_{1}\right]
$$

Hence, we find

$$
\begin{aligned}
Y_{0}= & \frac{4 \mathrm{~B}_{\phi}\left(\mu \mathrm{m} / \mathrm{B}_{0}\right)^{1 / 2}}{\mathrm{~N}-l t}\left\{[1-2 \arcsin (\alpha) / \pi]\left(\sigma_{0} / \Delta_{0}\right)^{1 / 2} \arctan \left(\sigma_{0}^{1 / 2}\right)\right. \\
& \left.+[1+2 \arcsin (\alpha) / \pi] g\left(\sigma_{1}, \Delta_{0} \Delta_{1}\right) / \Delta_{1}^{1 / 2}\right\}
\end{aligned}
$$

The integral for $Y_{1}$ is the same except that the first integral is now evaluated at $\eta=\Delta_{1} / \Delta_{0}$ and $\sigma=\sigma_{0}$, while the second is evaluated at $\eta=1$ and $\sigma=\sigma_{1}$. Thus, 


$$
\begin{aligned}
Y_{1}= & \frac{4 \mathrm{~B}_{\varphi}\left(\mu \mathrm{m} / \mathrm{B}_{0}\right)^{1 / 2}}{\mathrm{~N} \cdot 1 \mathrm{t}}\left(11 \cdot 2 \arcsin (\alpha) / \pi \mid g\left(\sigma_{0}, \Delta_{1} / \Delta_{0}\right) / \Delta_{0}^{1 / 2}\right. \\
& +\left[1+2 \arcsin (\alpha) / \pi \mid\left(\sigma_{1} / \Delta_{1}\right)^{1 / 2} \arctan \left(\sigma_{1}^{1 / 2}\right)\right\} .
\end{aligned}
$$

We have now determined each of the quantities in the expression for the trapping probability of Eq. (66). The calculation of the Poisson brackets leads to very complicated expressions, so at this point we make further simplifying approximations applicable to stellarators and tokamaks independently.

\section{Trapping probability in stellarator}

In this section we derive the formulas for the trapping probability in the small- $\alpha$ limit applicable to stellarators. We do not set $\alpha$ to zero, since this would give us only the previous results of Ref. 10. Instead we obtain the corrections through first order in $\alpha$. These corrections are important in the large-electric-potential limit, when the dominant drift is in the $\theta$ variable, because the $\theta$-variation of $\alpha$ then gives a significant change of the potential well depth. This will be seen more clearly in the comparisons with numerical experiments in the next section.

Through first order in $\alpha$ we replace the relative magnetic field variations of Eqs. (80) by

$$
\Delta_{(0,1)}=\varepsilon_{\mathrm{r}} \mp \pi \varepsilon_{\mathrm{r}} \alpha / 2+O\left(\alpha^{2}\right)
$$

Thus,

$$
\Delta_{0} \Delta_{1}=1-\pi \alpha .
$$


This differs from unity by a small amount, so 9 in Eq. (86) may be expanded as discussed in App. A. The lowest-order term of this expansion gives a term exalctly like the first term in the braces in Eq. (86) except that the index 0 is replaced by 1 . Since the appearance of $\alpha$ in these two terms is of opposite sign, upon adding one obtains simply twice the result obtained by setting $\alpha$ to zero in either of the terms. That is, $\Delta_{0,1}$ are replaced by $\varepsilon_{\mathrm{T}}$, and $\sigma_{0,1}$ are replaced by

$$
\bar{\sigma} \equiv 2 \varepsilon_{\mathrm{r}} /\left(1-\varepsilon_{\mathrm{r}} \cos (\theta)-\varepsilon_{\mathrm{r}}\right)
$$

In first correction to the expansion of App. A it is consistent to replace $\Delta_{1}$ by $\varepsilon_{r}$ and $\sigma_{1}$ by $\bar{\sigma}$. Ultimately, we obtain

$$
Y_{(0,1)}=\bar{Y} \mp \frac{1}{2} \Delta Y,
$$

where

$$
\bar{Y}=\frac{8 B_{\varphi}\left(\mu \mathrm{m} / B_{0}\right)^{1 / 2}}{N \cdot l_{t}}\left(\sigma / \varepsilon_{r}\right)^{1 / 2} \arctan \left(\sigma^{1 / 2}\right)
$$

and

$$
\Delta Y=\frac{4 \pi \alpha B_{\phi}\left(\mu m / B_{0}\right)^{1 / 2}}{\left(N-l_{t}\right)(1+\bar{\sigma}) \varepsilon_{\mathrm{r}}^{1 / 2}}\left[^{3 / 2} \arctan \left(\sigma^{1 / 2}\right)+(\bar{\sigma} / 2)(1+\ln \mid 16 / \pi \alpha l)\right]
$$

The energies $E_{0}$ and $E_{1}$, also needed for the probability (66) are given by

$$
E_{(0,1)}=\bar{E} \mp \frac{1}{2} \Delta E_{x}+O\left(\alpha^{2}\right) .
$$

The quantity $\Delta \mathrm{E}_{\mathrm{x}}$ is given exactly by $\mathrm{Eq}$. (79). The quantity $\overline{\mathrm{E}}$ is found from averaging Eqs. (77) and (78b) to lowest order in $\alpha$ :

$$
\overline{\mathrm{E}}=e \Phi+\mu B_{0}\left[1-\varepsilon_{\mathrm{t}} \cos (\theta)+\varepsilon_{\mathrm{r}}\right]
$$


Thus, through first order in $\alpha$ the trapping probability from Eq. (66) is

$$
P_{r}=\frac{f \cdot \Delta f}{1+f / 2+\Delta f / 2}
$$

where

$$
f \equiv[\bar{E}, \bar{Y}] /\left(2 \pi \mu B_{0} \varepsilon_{t} \sin (\theta)+\mathbb{N}\right)
$$

and

$$
\Delta \mathrm{f} \equiv([\Delta \mathrm{E}, \overline{\mathrm{Y}}]+[\overline{\mathrm{E}}, \Delta \mathrm{Y}]) /\left[4 \pi \mu \mathrm{B}_{0} \varepsilon_{\mathrm{t}} \sin (\theta)+\mathbb{N}\right]
$$

The evaluation of the trapping probability is straightforward but tedious, so we do not present the details of the algebra here. For numerical evaluation we found it simpler to evaluate the terms and combine them numerically.

\section{E. Trapping probability in rippled tokamak}

In tokamaks $l=0$, and the ripple is usually very small, of the order of $10^{-3}$. This allows one to neglect the ripple variation of the magnetic field in the denominator of the momentum $P_{\varphi}=m u B_{\Phi} / B$. This amounts to taking the limit of $\sigma \rightarrow 0$ in Eqs. (86) and (87). This limit was given previously in Sec. IVB of Ref. 10. The resulting formulas for the separaix actions are

$$
Y_{0}=\frac{8 B_{0}\left(\mu m \Delta_{0} / B_{0}\right)^{1 / 2}}{N\left(1-\varepsilon_{1} \cos (\theta)\right)}\left(1-\frac{2}{\pi} \arcsin (\alpha)+\left(1+\frac{2}{\pi} \arcsin (\alpha)\right) \sqrt{\frac{\Delta_{1}}{\Delta_{0}}} f(n)\right)
$$

and

$$
Y_{1}=\frac{8 B_{\phi}\left(\mu m \Delta_{1} / B_{0}\right)^{1 / 2}}{N\left(1-\varepsilon_{t} \cos (\theta)\right)}\left(1+\frac{2}{\pi} \arcsin (\alpha)+\left(1-\frac{2}{\pi} \arcsin (\alpha)\right) E(n)\right)
$$


with

$$
f(n)=E(n) \cdot\left(1 \cdot n^{2}\right) K(n)
$$

where

$$
n^{2} \equiv \Delta_{0} / \Delta_{1}
$$

and $K$ and $E$ are the complete elliptic integrals of the first and second kinds. To obtain the trapping probability one must insert these results for the separatrix actions along with (77) and (78b) for the separatrix energies into Eqs. (71) and (72). We did not carry out this algebraic calculation as it was simpler to evaluate only the derivatives and then compose the probabilities numerically.

\section{F. Analysis of different approaches}

Our calculation of the trapping probability is valid provided the drift frequency is small compared with the frequency for oscillation in a ripple well (for locally trapped particles) or traversal of ripple wells (for locally untrapped particles). There are now several other calculations (Refs. $1,7,8,10$ ) of the trapping probability. All of these calculations arrive at the conclusion that the trapping probability is a ratio of energy differences or, equivalently, when there is no potential, magnetic field strengths, since $\mathrm{V}=\mu \mathrm{B}$. The differences in the calculations arise in the approximations made in calculating these energy differences. Before proceding with the comparisons with numerics, it is useful to compare the various analytical calculations, so that we can understand how the differences arise.

\section{Comparison with Goldston and Towner}

The work of Goldston and Towner ${ }^{7}$ was geared towards the calculation of the trapping probability in tokamaks. In this case $\alpha$ can be large, so that the wells are highly nonsinusoidal. Goldston and Towner obtained 


$$
P_{G T}=\frac{R \varepsilon_{r}}{4 \pi \varepsilon_{l}\left(\mu B_{0}\right)^{1 / 2} t \sin (\theta)}\left\{T \frac{d\left(\ln \varepsilon_{r}\right)}{d t} \cdot \frac{p}{\varepsilon_{r} N} \frac{d}{d t}\left|\varepsilon_{l} \sin (\theta)\right|\right\}
$$

with $\mathrm{R}$ being the major radius of the torus, the functions $\mathrm{T}$ and $p$ given by

$$
T=\frac{16 \sqrt{2\left(1-\alpha^{2}\right)}(\pi / 2-\arcsin (\alpha)) \varepsilon_{\mathrm{r}}}{\pi \Delta_{0}}
$$

and

$$
p=8 \pi(\pi / 2 \cdot \arcsin (\alpha))
$$

and $\Delta_{0}$ given by Eq.(72a). (This definition of $T$ corrects a misprint on p. 293 of Ref. 7.) The time derivative in Eq.(98) is a derivative along the drift trajectory,

$$
\frac{d}{d t}=\dot{\theta} \frac{\partial}{\partial \theta}+\dot{\rho} \frac{\partial}{\partial \rho}
$$

where

$$
\dot{\theta}=\frac{1}{m p \Omega_{0}}\left\{e \frac{d \Phi}{d \rho}+\mu B_{0}\left[\frac{d \varepsilon_{r}}{d \rho}-\frac{d \varepsilon_{l}}{d \rho} \cos (\theta)\right]\right\}
$$

is the bounce-averaged angular drift for a near-separatrix particle, and

$$
\dot{\rho}=-\frac{\mu B_{0}}{m R_{0} \Omega_{0}} \sin (\theta)
$$

is the bounce-averaged radial drift for a near-separatrix particle. The new variables introduced in these equations are explained in detail in Sec. VA. 
Goldston and Towner also assumed that the ripple amplitude is small, and so they made the approximations described in Sec. IVE. These approximations, accurate for tokamaks, are often invalid for stellarators, where the ripple amplitude can be appreciable. i.e., the parameter $\sigma$ can easily be as large as 0.7 . It may also be noted that the results of Goldston and Towner were left in the form of definite integrals, which were then approximated. It was not recognized that the various terms were just derivatives of the separatrix-enclosed actions.

2. Comparison with Cary, Hedrick, and Tolliver

In this work ${ }^{10}$ the ripple-averaged adiabatic invariants were introduced. These invariants were calculated by expansion in the poloidal motion per ripple period. Because the poloidal angle changes by approximately $2 \pi t / \mathrm{N}$ in following a magnetic field line through one ripple period, this expansion assumes that $t / \mathbb{N}$ (and, hence, $\alpha$ ) are small in addition to the guiding-center drift per ripple period being small. Such an approximation may be necessary for the calculation of ripple-averaged invariants for locally untrapped particles, because standard adiabatic theory requires ${ }^{11}$ the trajectories to be nearly (i.e., in lowest order) closed loops in phase space, and only when $\theta$ is constant can the locally untrapped trajectories be closed loops, because then the potential energy is exactly periodic in the remaining variable, the toroidal angle $\varphi$.

Given an adiabatic theory for all classes of trajectories, the trapping probability can be simply expressed in terms of the relative rates of change of the separatrix actions as was shown in early work on the theory of separatrix crossing. ${ }^{16}$ The resulting formulas are obtained from those derived here by eliminating the terms $\Delta \mathrm{f}$ in Eq. (97). The resulting trapping probabilites are always in the physical range $(0,1)$. Unfortunately, because the expansion in $t / N$ is also made, the results of Cary, Hedrick, and Tolliver should not be as accurate as the present results, because in the present analysis all of the the motion along 
field lines is taken into account exactly. Only the drift motion is treated pernurbatively in the calculation of the trapping probability. But, in the present work we do not give ripple averaged invariants for the case of large $t / N$. This remains an area for future work.

\section{Comparison with Nagornij and Yavorskij}

In this work, ${ }^{8}$ the trapping probability was defined to be the appropriate ratio of magnetic field strength differences, since the potential energy was ignored. The calculation provided the differences due to drift basically in terms of Poisson brackets of action. However, in the action integrals, the denominator in $p_{\varphi}=m u B B_{\varphi} / B$, which gives a lengthening of the field line when the magnetic field is weak, was ignored. Hence, the results of Ref. 8 are valid only for small $\varepsilon_{\mathrm{r}}$. In addition, the authors miss the factor $l+/ N$ seen in Eq. (74), which comes from the transformation from poloidal angle $\theta$ to field-line angle $\beta$.

Even with these facts taken into account, we do not agree exactly with their final Eq. (55), in getting to which they used the fact that $\alpha$ is small. Possible additional approximations are not visible, since the intermediate steps were not published. It is somewhat disturbing that their final result contains a nonperiodic (in fact, linear) function of the poloidal angle.

For numerical comparison, where we assumed that the magnetic surfaces are circular (cf Sec. VA for definitions), we first define 21

$$
\begin{aligned}
P_{N Y} & =\frac{4\left(\varepsilon_{\mathrm{r}} \gamma_{\mu}\right)^{1 / 2}}{\pi \varepsilon_{t}}\left\{\frac{1}{\varepsilon_{\mathrm{r}}} \frac{\mathrm{d} \varepsilon_{\mathrm{r}}}{\mathrm{d} \rho}\left[1+\frac{t}{N}\left(|\theta|-\pi+\frac{\pi}{4} \ln (16 / \pi \alpha)\right) \cot (|\theta|)\right]\right. \\
+ & \left.\frac{2 l}{N} \frac{\mathrm{dt}}{\mathrm{d} \rho}+\frac{2 \gamma_{\Phi}}{\gamma_{\mu} \Phi_{0}} \frac{\mathrm{d} \Phi}{\mathrm{d} \rho}\left[1+\frac{t}{N}\left(|\theta|-\pi+\frac{\pi}{8 \varepsilon_{\mathrm{r}}} \ln (16 / \pi \alpha)\right) \cot (|\theta|)\right]\right\},
\end{aligned}
$$


the trapping probability derived by $V$. Nagornij in the same fashion but including the effects of an electrostatic potential. This result differs from the published ${ }^{8}$ result in that the published result did not contain the terms linear in $d \Phi / d \rho$ and $d t / d \rho$, and $N$ is replaced by $N-l t$.

\section{Comparison with Kovrizhnykh and Shasharina}

This work' starts from the previously noted result of separatrix-crossing theory that the trapping probability depends on the relative rates of change of the separatrix enclosed areas. This work was the first to introduce a model for the variation of the magnetic field strength along a field line so that the action integrals could be given explicitly for arbitrary values of $\alpha$. In this model the field is approximated by a single sinusoid plus a constant portion, which is inaccurate in the region where the constant-B approximation is made. The present calculation improves on this idea by using pieced sinusoids. Also the work of Ref. 1, like two of the other calculations, ${ }^{7,8}$ neglects the factor of $1 / \mathrm{B}$ in the action integral, so the results are not accurate for large values of $\varepsilon_{r}$. In addition, the factor $\left(1-l_{t} / N\right)$ of Eq. (65) is not present.

The the magnetic field, $B=B_{T}\left\{1-\Delta_{0} \cos \left[k_{0}\left(x-x_{m n}\right)\right]\right\}$, with $B_{T} / B_{0}=1-\varepsilon_{t} \cos \left(\theta_{0}\right)$, used in Ref.1 had a toroidal modification of the ripple amplitude, since $\mathrm{B}_{\mathrm{T}}$ is a factor of the entire field. To be consistent with the present calculation, one must replace 1 by 2 in the brackets of the definition of $C$ in Eq. (12) of Ref. 1.

\section{Summary}

All trapping probability calculations relate the trapping probability to the relative flux of trapping trajectories near the transition point. In the present work the validity of this argument is considered in detail and shown to rely on the ratio of poloidal to ripple frequencies being irrational, which is the case nearly everywhere when there is shear. The 
relative flux is related, equivalently, to either the energy ranges of trapping and reflecting orbits or to the rate of change of the phase space enclosed by the various separatrices.

The major differences arise in the choice of the lowest-order system, the model magnetic field used, and subsequent approximations. In Refs. 1, 7, 8, and the present calculation the lowest order motion was along a field line ignoring drift. In Ref. 10 the lowest order motion ignored also the change of the poloidal angle due to finite rotational transform in order to develop adiabatic invariants for the locally passing trajectories. In all calculatiuns except the present and Ref. 10, the 1/B factor was ignored in the action integral. This factor is important when the ripple is large. In Ref. 7 the trapping probability was implicitly assumed to be small, while in Refs. 8 and 10, $\alpha$, which measures the well distortion away from sinusoidal, was assumed to be small. In Ref. I a mord is introduced to eliminate the need for these approximations. In the present work, an improved model that is a uniform approximation is introduced.

In summary, the present calculation improves on the previous in one or another of the following ways. (1) The trapping probability is not assumed to be small. (2) The ripple strangth is not assumed to be small. (3) The rotational transform per field period is not assumed to be small. (4) A uniform approximation for the magnetic field strength along a field line is used. 
V. Comparison of analytic theory with numerical simulation

As discussed in the previous section, in the calculation of the trapping probabilities here and elsewhere approximations are made, because the exact irajectories cannot be found. If the small parameter of each of these calculations were indeed infinitesimal, then the calculations would have to be correct. (We have used this fact to check our that our calculation was done correctly.) But in typical situations these small parameters can be fairly large. For example, the quantity $\sigma$ can be as large as 0.7 in present machines, but it is assumed to be small in Refs. 1, 7, and 8 . All of the calculations assume the time scales of the drift and the bounce motion to be widely separated, but this too can begin to fail for realistic parameters, as we will see in this section.

The purpose of this section is to show comparisons of the present theory with numerical integrations of the full guiding-center equations in cases for typical parameters, which may be near breakdown of the time-scale separation. We compare the numerical results not only with our own theory, but also with the previously given theories. We will see that for a typical stellarator configuration without ambipolar potential, the time-scale separation is fairly large and, as a result, the present theory does well at predicting the transition probability. However, for ions in ambipolar potentials of the order of their energy, the time-scale disparity is smaller, and so the agreement between the analytic transition probability and the numerical result is not so good. Nevertheless, the analytic theory yet gives a reasonable value of the transition probability.

A direct comparisons with transition processes in tokamaks was done previously in Ref. 7, where it was shown that the approximations made by Goldston and Towner allowed for accurate prediction of the transition probability for a typical tokamak case. We have performed analogous calculations here so that our results can be compared with those of Goldston and Towner for tokamak parameters. 
A. Typical parameters and time-scale separation

The parameters of the numerical integrations for the stellarator case are those of Ref. 10, which are ATF-like ${ }^{22}$ (Advanced Toroidal Facility). We briefly describe these parameters again here. The vector potential, $A_{\varphi}=-t_{0} \psi-t_{1} \psi^{2} / 2 \psi_{e}$, where $\psi_{e}$ is the toroidal flux at the plasma edge, has shear; the rotational transform rises from $t_{0}$ at the center to $t$ )$_{0}+t_{1}$ at the plasma edge. The values $t_{0}=0.35$ and $t_{1}=0.55$ are consistent with ATF. The edge toroidal flux is given by $\psi_{\mathrm{e}}=\frac{1}{2} \mathrm{~B}_{0} \mathrm{a}^{2}$, where $\mathrm{a}$ is the minor radius of the plasma. The ripple amplitude and toroidicity functions are chosen to be $\varepsilon_{\mathrm{r}}=\delta_{\mathrm{r}} \rho^{2}$ and $\varepsilon_{\mathrm{t}}=\delta_{\mathrm{t}} \rho$, where $\rho \equiv\left(\psi / \psi_{e}\right)^{1 / 2}$ is the normalized mean radius of a flux surface, and $\delta_{t}=a / R_{0}$ with $R_{0}$ being the major radius on axis. The edge values $\delta_{r}=0.21$ and $\delta_{t}=0.14$ are consistent with ATF. The electrostatic potential is also assumed to have parabolic form: $\Phi=\Phi_{0} \rho^{2}$. Finally, we choose $B_{\varphi}=B_{0}$ to be constant, consistent with having a vacuum magnetic field.

For the tokamak case, we use the exponential model for the ripple amplitude (see [6]), $\varepsilon_{\mathrm{r}}=\delta_{\mathrm{r}} \exp \left(\mathrm{Nb} / \delta_{\mathrm{r}}\right), b=\sqrt{\rho^{2}+\mathrm{L}^{2}+2 \rho L \cos (\theta)}$, where $b$ is the relative (to minor radius) shift of the center of the ripple from the plasma center. The geometrical parameters are $t_{0}=1, t_{1}=0.67, N=12, \delta_{r}=0.031, \delta_{l}=0.14$.

We integrate in dimensionless variables for which the unit of length is the minor radius, and the unit of time is the inverse of the gyrofrequency $\Omega_{0}=q B_{0} / m c$. A trajectory has three dimensionless energy parameters: $\gamma_{\mu} \equiv \mu B_{0} / m\left(\Omega_{0} a\right)^{2}, \gamma_{E} \equiv E / m\left(\Omega_{0} a\right)^{2}$, and $\gamma_{\Phi} \equiv q \Phi_{0} / \mathrm{m}\left(\Omega_{0} \mathrm{a}\right)^{2}$. The parameter $\gamma_{\mu}$ is the square of the ratio of the gyroradius at the machine center to the minor radius and is taken to have the typical value of $1.81 \times 10^{-4}$. The parameter $\gamma_{E}$ has the same interpretation, with perpendicular energy replaced by total (kinetic plus potential) energy. The parameter $\gamma_{\Phi}$ has the analogous interpretation with particle energy replaced by edge potential energy. For $1.7 \mathrm{keV}$ protons in a magnetic field of $1 \mathrm{~T}$ in a machine of minor radius $30 \mathrm{~cm}, \gamma_{E}=2 \times 10^{-4}$. 
Adiabatic theory requires that the particles make many bounces in the locally trapped state before they become untrapped. The number of the bounces in the local magnetic well, $\mathrm{M}$, of the transitional particle can be estimated as,

$$
M \approx \frac{\Delta \theta}{\dot{\theta}_{\mathrm{dr}} \mathrm{T}_{\mathrm{b}}}
$$

where $\Delta \theta$ is a poloidal width of the locally trapped orbit, which has an order of unity for stellarator (and $\Delta \theta \approx 1 / \alpha$ for tokamaks), $\dot{\theta}_{\mathrm{dr}}$ is the poloidal drift velocity, and $T_{\mathrm{b}}$ is the bounce period $\left(2 \pi / \omega_{b}\right.$ with $\omega_{b}$ from Eq. (20a)). The poloidal drift velocity and the bounce period are given approximately by

$$
\dot{\theta}_{\mathrm{dr}} \approx \frac{1}{\mathrm{~m} \Omega_{0} \mathrm{a}^{2} \rho} \frac{\partial}{\partial \rho}\left\{\mathrm{e} \Phi+\mu \mathrm{B}_{0}\left[\varepsilon_{\mathrm{r}}-\varepsilon_{\mathrm{t}} \cos (\theta)\right]\right\}
$$

and

$$
\mathrm{T}_{\mathrm{b}} \approx \frac{2 \pi \mathrm{R}_{0}}{\mathrm{~N} \rho\left(\mu \mathrm{B}_{0} \delta_{\mathrm{r}}\right)^{1 / 2}}
$$

We these relationships to the above formula, we find the number of bounces,

$$
M=\frac{N \varepsilon_{t} \delta_{r}^{1 / 2}}{4 \pi \gamma_{\mu}^{1 / 2}\left(\gamma_{\Phi} / \gamma_{\mu}+\delta_{r}-\delta_{t} \cos (\theta) / 2 p\right)}
$$

In the absence of an ambipolar potential at a location halfway out in minor radius with the paramaters noted above, $M$ is of the order of 10 . Thus, we expect our trapping probability to do well in this case. However, with a potential of the order of the perpendicular energy, the number of bounces drops to two or three. Hence, we expect our transition theory to be marginally valid. Surprisingly we find that cyen for these cases our theory does a fair job. 


\section{B. Numerical method}

To determine the trapping probability, trajectories were integrated with the Hamiltonian guiding center equations. The trajectories were started at $\theta=0$ for various values of $\varphi$ with fixed initial value of the energy and the first-order-corrected adiabatic invariant. (Explicit formulas for this invariant are given in App. B.) The value of the initial parallel velocity was determined from energy conservation, while the initial value of the flux variable $\psi$ was determined from chosen value of the corrected adiabatic invariant: For an initial value of $\varphi$ the equation $J_{i}=J^{1}(\psi, \theta=0, \varphi, E, \mu)$ is solved by Newton's method to determine the initial value of $\psi$.

Integration of the trajectories allowed us to determine whether a trajectory reflects or becomes trapped. A trajectory was integrated until the derivative $\dot{\chi}$ changed sign, indicating reflection from a second local maximum. The trajectory was then integrated until one of two things happened. If a second sign change of $\dot{\chi}$ occurred, the trajectory had become trapped in a local well like those between points $b$ and $c$ in Fig. 6. Otherwise the value of $\chi$ changed by more than $2 \pi$, indicating the trajectory had passed over the local maximum upon returning like those trajectories between points $a$ and $b$.

By successive bifurcations we determined the range of initial values of $\varphi$ for which trajectories reflect or become trapped. Ultimately we found two initial values, $\varphi_{T 1}$ and $\varphi_{\mathrm{T} 2}$, such that trajectories launched between $\varphi_{\mathrm{T} 1}$ and $\varphi_{\mathrm{T} 2}$ (modulo $2 \pi / \mathrm{N}$ ) become trapped, while trajectories launched between $\varphi_{\mathrm{T} 2}$ and $\varphi_{\mathrm{T} 1}(\operatorname{modulo} 2 \pi / \mathrm{N})$ reflect. The uncertainty of $\varphi_{\mathrm{T} 1}$ and $\varphi_{\mathrm{T} 2}$, determined by the level of bifurcation at which one stops, was reduced to less than $10^{-4}$. Upon examining Fig. 6 we note that for positive passing trajectories $(u>0)$ the trajectory launched at $\varphi_{\mathrm{T} 1}$ asymptotes into point $c$, while the trajectory launched at $\varphi_{\mathrm{T} 2}$ asymptotes into point $b$ after reflecting. For negative passing trajectories these roles are reversed. 
The last task is to calculate the relative flux of these two regions. As noted in Sec II, the trapping probability is given by the relative flux of trajectories in ranges $\delta \mathrm{E}$ and $\delta \mathrm{J}$ that become trapped. In the original coordinates this flux is

$$
F=\int d \varphi d p_{\varphi} d \psi \dot{\theta}
$$

The integral over $P_{\phi}$ is change through two variable substitutions, first $d E=d p_{\varphi}\left(\partial h / \partial p_{\varphi}\right)=d p_{\varphi} \dot{\varphi}$ and second $d J=d \psi\left(\partial J^{1} / \partial \psi\right)$. Thus we obtained the flux of trajectories that become trapped as

$$
\mathrm{F}_{\mathrm{r}}=\delta \mathrm{J} \delta \mathrm{E} \int_{\varphi_{\mathrm{T} 1}}^{\varphi_{\mathrm{T} 2}} \mathrm{~d} \varphi \frac{\dot{\theta}}{\dot{\varphi}} / \frac{\partial \mathrm{J}^{1}}{\partial \psi}
$$

while the total flux is obtained by integrating the same quantity over a full period. The ratio gives the numerical transition probability.

In previous numerical work trajectories were chosen at random in the midplane, and the fraction becoming trapped was said to be the trapping probability. This corresponds to setting the integrand in Eq. (101) to a constant. The denominator,

$$
\frac{\partial J^{1}}{\partial \psi}=\frac{\partial}{\partial \psi}\left(J_{0}(\Psi, \theta, E)+\varepsilon_{a} J_{1}(\Psi, \theta, \varphi, E)\right)
$$

is nearly a constant when adiabatic theory is valid, so that the correction is small. However, the other term,

$$
\frac{d \theta}{d \varphi}=t+\frac{\partial}{\partial \theta}\left(\frac{u B_{0}}{\Omega_{0} \delta_{t} B}\right)
$$


(from Ref. 10) is nearly constant only when the second term can be ignored, which is typically possible only when the ripple amplitude is small (as it is in tokamaks) relative to the particle energy at midplane, so that the parallel velocity $u$ is nearly constant at fixed energy and poloidal location.

Lastly we must assign a trapping angle and value of the flux to any given numerical run. We could use the adiabatic theory of Ref. 10, but we chose not to do this in order to eliminate the number of assumptions in the numerical experiments. The chosen angle should be in the middle of the trapping region shown in Fig. 6. Were this figure precise, that would be the angle of point $c$, which separates a trapping trajectory from a reflecting trajectory. However, the actual separating trajectory never gets to the point $c$, it only asymptotes to this point. Furthermore, in this process this trajectory (and others in this exponentially small class) undergoes large drifts that carry it far from the trapping region of most trajectories.

To get around this problem we first search for the halfway trajectory. This is the trajectory that is launched at point $\varphi_{\mathrm{h}}$ chosen such that half of the trajectories trapping or reflecting in a given period have initial value of $\varphi$ less than $\varphi_{h}$, and so, of course, half have initial value greater. This condition is

$$
\int_{\varphi_{\mathrm{T} 1}}^{\varphi_{\mathrm{h}}} \mathrm{d} \varphi \frac{\dot{\theta}}{\dot{\varphi}} / \frac{\partial \mathrm{J}^{\mathrm{l}}}{\partial \psi}=\frac{1}{2} \int_{\varphi_{\mathrm{T} 1}}^{\varphi_{\mathrm{T} 1}+2 \pi} \mathrm{d} \varphi \frac{\dot{\theta}}{\dot{\varphi}} / \frac{\partial \mathrm{J}^{\mathrm{l}}}{\partial \psi} .
$$

This trajectory passes through the center of the trapping region and is not an asymptote. It is integrated until it first reflects. Then it is integrated back to where is crossed its last local maximum. Finally, it is irtegrated forward until $\chi$ has increased by $\pi$. The value of $\psi$ and 
$\theta$ for this trajectory at this point is used in the analytic expressions for the transition probiility for the entire class of trajectories.

\section{Comparison of numerical results and analytical calculations}

We now proceed to compare our numerical calculation of the trapping probability with the various analytical calculations. For the stellarator case (Figs. 8-10) we use parameters close to those of Figs. 4, 7, and 8 of Ref. 10. Specifically, we take $\gamma_{\mu}=1.81 \times 10^{-4}$ and the other parameters the same. In these examples the theory of Ref. 10 predicts that the majority state is the negative passing state, so that particles trap at negative values of $\theta$.

We begin by illustrating, in Fig. 8, the error incurred by not holding the adiabatic invariant constant through first order for the ensemble of launch conditions. For this figure the parameters are $\gamma_{E}=2 \times 10^{-4}$ and $\gamma_{\Phi}=0$, the same as for Fig. 3. The numerically obtained transition probability for an ensemble with constant zeroth-order adiabatic invariant is labeled $P_{0}$, while the numerically obtained transition probability for an ensemble with constant first-order-corrected adiabatic invariant is labeled $P_{1}$. This figure shows that using constant lowest-order adiabatic invariant gives order-unity oscillations in the numerical transition probability as predicted in Sec. III-A. Hence, in what follows, our numerical results are based on an ensemble of launch points with constant first-ordercorrected adiabatic invariant.

For Fig. 9 the parameters are the same as for Fig. 8. In this case of no electrostatic potential, the transition curve (shown in Fig. 3) has only a finite range of poloidal angle inside the physical region $(p<1)$. The numerical trapping probabilities are shown as points. The analytical calculations are labeled curves: GT (Goldson and Towner) denotes the result (98), CHT (Cary, Hedrick and Tolliver) denotes the results of Ref. 10, NY (Nagomij and Yavorskij) denotes the result (100), KS (Kovrizhnykh and Shasharina) 
denotes the results of Ref. 1 , and CS (Cary and Shasharina) denotes the present results. This figure shows that for $\theta$ near $-3 \pi / 4$ all of the theories, except that of Goldston and Towner, accurately give the trapping probability. As the main difference is that Goldston and Towner use a model for the evaluation of their integrals, we see that this model is invalid. For trapping near $\theta=-\pi / 4$, only the present calculation and that of Ref. 10 are accurate. Hence, we deduce that the neglect of the $1 / \mathrm{B}$ factor in the action integrals is invalid. It is clearly seen in this picture that over the entire range, the present calculation gives best result for the trapping probability.

The numerical trapping probability is seen to have oscillations that are not present in any of the analytical results. These oscillations are associated with the finite accuracy of the adiabatic theory. We have verified this in our numerical simulations by observing that they decrease with decreasing adiabatic parameters (gyroradius to scale length and rotational transform per field period).

For Fig. 10, a positive electrostatic potential $\gamma_{\Phi}=2 \times 10^{-4}$ has been introduced. To have the same kinetic energy half way out in $\psi$, the total energy is chosen to be $\gamma_{E}=3 \times 10^{-4}$. Here it is seen that the general shape of the transition probability curve is obtained by all calculations except that of Ref. 10. Hence, it is deduced that the associated expansion in $t / \mathbb{N}$ must not be good. Again we argue that the present calculation is superior. It is everywhere more accurate than the calculations of Ref. 1 and 7 , and its accuracy is rivaled for small $1 \theta i$ only by the calculation of Ref. 8.

For Fig. 11, a negative electrostatic potential $\gamma_{\Phi}=-2 \times 10^{-4}$ has been introduced. To have the same kinetic energy half way out in $\psi$, the total energy is chosen to be $\gamma_{E}=1 \times 10^{-4}$. In this case, the two best results are the present and that of Ref. 1. The present result is accurate to with $\Delta P=0.1$ over most of the angular range. The inaccuracy appears to be due to a breakdown of adiabaticity. A signature of this is that the oscillations 
in the numerical results are large, as we noted earlier. To verify this, we kept track of the number of ripple periods crossed before trapping. For those trajectories trapping near $\theta=\pi / 2$, only two ripple periods are crossed. So few periods are crossed because these trajectories end up being very close to the separatrix at launch, and because the negative potential increases the speed at which these trajectories travel towards the trapping point.

This vanishing of the numerically calculated trapping probability (at $\theta$ near $-\pi / 4$ ) implies that the flux of trajectories becoming trapped at more negative values of $\theta$ is negative, i.e., trajectories are in fact detrapping. Hence, both trapped and negative passing trajectories are converting to positive passing trajectories, so that the positive passing state is the majority state. This implies that trapping occurs for these trajectories above the midplane when the magnitude of $\theta$ is less than $\pi / 4$.

This shows that the theory of Ref. 10 poorly predicts the majority state for these values of the parameters. The reason for this breakdown appears to be the following. For these parameter values the locally trapped contours are very nearly tangent to the transition contour. Thus, a slight shift of these invariant contours, as illustrated in Fig. 12, can change these contours from incoming on the bottom to incoming on the top. This indicates the need for a more accurate theory of the ripple-averaged invariants.

We argue that the large relative inaccuracy for $\theta$ in the range $[-\pi / 2,-\pi / 4]$ is not so important. In this range the transition probability is small, so the process is not important there. Thus, we believe that the results shown in Figs. 8-10 indicate that the present adiabatic theory with its calculated transition probabilities provide a reasonably accurate basis for the development of a theory of transport.

We also have investigated the case with parameters typical for the tokamak configuration, when the helical modulation is much smaller then the toroidicity, $\delta_{r} \ll \delta_{1}$. The corresponding results are shown on Fig. 13, where the parameters are $\gamma_{\Phi}=0$, 
$\gamma_{E}=1 \times 10^{-4}, \gamma_{\mu}=0.93 \times 10^{-4}$. Since for the tokamak case we have only two models 10 compare, we plot our analytical result given by Eqs(97a-b) (CS) and that presented in Ref. $7(\mathrm{GT})$. The agreement of both models with the numerical data is very good, which means. that the model of Goldston and Towner works well for small ripple, as is the case in tokamaks. 
VI.

Summary and conclusions

The present work ties together and makes more rigorous many of the previous calculations concerning particle transitions in toroidal magnetic confinement schemes with local magnetic wells. We have shown how to define the transition probability unambiguously. We have provided a new analytic calculation of the transition probability that improves upon the existing results. Finally, we have shown by direct comparison with numerical integration that the present results do provide an improvement over previous results for realistic stellarator parameters, and are sufficiently accurate.

Our results also point to the importance of understanding adiabatic theory for this system. The adiabatic invariants determine the range of the particle motion. The adiabatic Hamiltonian determines the rate at which trajectories traverse these contours. We have seen in the last section, that the previous theory ${ }^{10}$ of the adiabatic invariants can incorrectly fredict the majority state of transitioning particles. Further work in this area is needed. 
Appendix A. Asymptotics of $g(\sigma, \eta)$ for $\eta$ near unity

For the stellarator and expansion of Sec. IV it is assumed that $\alpha$ is small, which implies that the second argument of $g$ is near unity. In this appendix we derive the expressions for 9 appropriate to this limit. In particular we calculate $g(\sigma, \eta)$ for $\eta$ near unity through first order in the difference $\eta-1$. The expansion of the action near the separatrix, though singular, is odd ${ }^{16}$ in the difference between the energy and the value on the separatrix to lowest order. This implies that we need calculate the expansion only for $\eta$ either greater or less than unity, and the other follows.

For $\eta$ greater than unity, the definitions (42)-(43) imply that

$$
g(\sigma, \eta)=\sigma \int_{0}^{\pi / 2} d z \frac{\left(\eta-\sin ^{2}(z)\right)^{1 / 2}}{1+\sigma \sin ^{2}(z)}
$$

To determine the asymptotics of this function, we will carry out three operations. The first is to determine the value at $\eta=1$. The second is to evaluate the derivative $\partial 9 / \partial \eta$ for $\eta$ near unity. The last is to integrate once to obtain 9 for $\eta$ near unity.

The value of 9 for $\eta$ at found by simple substitution. Thus,

$$
g(\sigma, 1)=\sigma \int_{0}^{\pi / 2} \mathrm{~d} z \frac{\cos (z)}{1+\sigma \sin ^{2}(z)}=\sigma^{1 / 2} \arctan \left(\sigma^{1 / 2}\right)
$$

The derivative has the form,

$$
\frac{\partial g}{\partial \eta}=\frac{1}{2} \sigma \int_{0}^{\pi / 2} \frac{d z}{\left[\eta-\sin ^{2}(z)\right]^{1 / 2}\left[1+\sigma \sin ^{2}(z)\right]}
$$




$$
\begin{aligned}
& =\frac{1}{2} \sigma \int_{0}^{\pi / 2} \frac{d z}{\left[\eta-1+\sin ^{2}(z)\right]^{1 / 2}(1+\sigma)} \\
& +\frac{\sigma^{2}}{2(1+\sigma)} \int_{0}^{\pi / 2} \frac{d z \cos ^{2}(z)}{\left[\eta-1+\cos ^{2}(z)\right]^{1 / 2}\left[1+\sigma \sin ^{2}(z)\right]}
\end{aligned}
$$

In the first integral after the second equals sign the substitution $z \rightarrow \pi / 2-z$ has been made. This integral is the complete elliptic of the first kind, and its asymptotics are well known. The second integral is not singular at $\eta=1$, so this may be directly substituted in. This gives an integral identical to that for $g(\sigma, 1)$. Thus, we find

$$
\frac{\partial g}{\partial \eta}=\frac{\sigma^{3 / 2} \arctan \left(\sigma^{1 / 2}\right)}{2(1+\sigma)}+\frac{\sigma}{2(1+\sigma)} \ln \left|\frac{16}{\eta-1}\right|
$$

for $\eta$ near unity.

The last step in this calculation is $\mathrm{t}$

:Eq. (A4) starting from unity where the value is given by Eq. (A2). This gives

$$
\begin{aligned}
g(\sigma, \eta) & \approx \sigma^{1 / 2} \arctan \left(\sigma^{1 / 2}\right)+\frac{\eta-1}{2(1+\sigma)}\left\{\sigma^{3 / 2} \arctan \left(\sigma^{1 / 2}\right)\right. \\
& +(\sigma / 2)[1+\ln |1 \sigma /(\eta-1)|]\}
\end{aligned}
$$

for $\eta$ near unity. As mentioned above, this result is valid to $O(\eta-1)$ for both $\eta$ gretaer than or less than unity. 
Appendix B. Corrections to the adiabatic invariant

To carry out the numerical determination of the transition probability, it was necessary to calculate the first-order correction (45) of the adiabatic Invariant for the standard stellarator model. This calculation is tedious and so relegated to this appendix. It is presented at all to allow future researchers results for comparison. It takes place in two steps: simplification of to a form without double integrals and reduction of this form to derivatives of incomplete elliptic integrals. We will derive the results for the positive passing case, then state the results for the other cases.

\section{B 1. Simplication to eliminate double integrals}

The integrations in Eq. (45) are more conveniently expressed in in terms of the variable $\chi$, which was the phase of the ripple. The transformation of the integrals to this variable is straightforward, because $\varphi=(\chi+l \theta) / \mathrm{N}$, and the integrations are at fixed $\theta$. However, the $\theta$-derivative changes under this transformation according to the usual rule,

$$
\left.\left.\frac{\partial p_{\varphi}}{\partial \theta}\right)_{\varphi}=\frac{\partial p_{\varphi}}{\partial \theta}\right)_{x} \cdot l \frac{\partial p_{\varphi}}{\partial \chi}
$$

where the subscript indicates what is being held constant in taking the derivative. Henceforth in this appendix all derivatives are with respect to the $(\psi, \theta, \chi)$ coordinates. That is, for example, $\chi$ is held constant in derivatives with respect to $\theta$. Substitution of this into Eq. (45) gives

$$
\begin{aligned}
g_{1}(\chi) & =-\frac{c}{2 e} \frac{\partial g_{0}}{\partial \theta} \frac{\partial g_{0}}{\partial \psi} \\
& +\frac{c}{e^{2}} \oint d \chi^{\prime} \frac{\partial p_{\varphi}}{\partial \psi}\left(\chi^{\prime}\right) \int_{\chi}^{\chi^{\prime}} d \chi^{\prime \prime}\left(\frac{\partial p_{\varphi}}{\partial \theta}\left(\chi^{\prime \prime}\right)-l \frac{\partial p_{\varphi}}{\partial \chi^{\prime}}\left(\chi^{\prime}\right)\right)
\end{aligned}
$$


Here the state subscript is left off the quantities, because no assumption about the state has yet been made.

We calculate each of the terms in Eq. (B2) in sequence. The first term we dela with is part of the integral:

$$
\begin{aligned}
g_{1 \mathrm{a}} & \equiv-\frac{l \mathrm{c}}{\mathrm{eN^{2 }}} \oint \mathrm{d} \chi^{\prime} \frac{\partial p_{\varphi}}{\partial \psi}\left(\chi^{\prime}\right) \int_{\chi}^{\chi^{\prime}} \mathrm{d} \chi^{\prime \prime} \frac{\partial p_{\varphi}}{\partial \chi}\left(\chi^{\prime \prime}\right) \\
& =-\frac{l \mathrm{c}}{\mathrm{eN^{2 }}} \oint \mathrm{d} \chi^{\prime} \frac{\partial p_{\varphi}}{\partial \psi}\left(\chi^{\prime}\right)\left[p_{\varphi}\left(\chi^{\prime}\right)-p_{\varphi}(\chi)\right] .
\end{aligned}
$$

In simplifying this term, we note the definition (15) implies that taking the difference $p_{\varphi}\left(\chi^{\prime}\right)-P_{\varphi}(\chi)$ eliminates $A_{\varphi}$ and leaves

$$
p_{\varphi}\left(\chi^{\prime}\right)-p_{\varphi}(\chi)=m u\left(\chi^{\prime}\right) B_{\varphi^{\prime}} / B\left(\chi^{\prime}\right)-m u(\chi) B_{\varphi} / B(\chi)
$$

The second term in Eq. (B4) is a constant in the integration of (B3) and so comes out of the integration:

$$
g_{1 \mathrm{a}}=\frac{l \mathrm{mcuB}_{\varphi}}{\mathrm{eBN}^{2}} \oint \mathrm{d} \chi^{\prime} \frac{\partial p_{\varphi}}{\partial \psi}\left(\chi^{\prime}\right)-\frac{l \mathrm{c}}{\mathrm{eN}^{2}} \oint \mathrm{d} \chi^{\prime}\left(\mathrm{et} / \mathrm{c}+\frac{\partial\left(\mathrm{muB}_{\phi} / \mathrm{B}\right)}{\partial \psi}\right) \mathrm{muB}_{\varphi} / \mathrm{B}
$$

The first integral with a factor of $1 / \mathbb{N}$ is the $\psi$-derivative of the lowest-order action. The first term coming from the second integral, after pulling out the factor of $-e t / c$ is relater to the lowest-order invariant ( 31 ) by discarding the vector-potential term. The last term can be written as a single derivative. These manipulations give

$$
g_{1 \mathrm{a}}=\frac{l \mathrm{mcuB}_{\varphi}}{\mathrm{eBN}} \frac{\partial g_{0}}{\partial \psi}+\frac{l t}{N} g_{0 u}-\frac{l \mathrm{~m}^{2} \mathrm{c}}{2 \mathrm{eN}^{2}} \frac{\partial}{\partial \psi} \oint \mathrm{d} \chi^{\prime}\left(\mathrm{uB}_{\varphi} / \mathrm{B}\right)^{2}
$$




$$
g_{0 u} \equiv\left\{\begin{array}{cc}
g_{ \pm 0} \mp \mathrm{eA}_{\varphi} / \mathrm{c} & \text { for passing particles } \\
g_{\mathrm{r} 0} & \text { for trapped particles }
\end{array}\right.
$$

is that part of the action from the term proportional to the parallel velocity in the expression (15) for the canonical momentum. This is all the action for trapped particles. We note that the last term in Eq. (B6) vanishes for trapped particles because the integration along the loop in the $+\chi$ direction is canceled by the integration int the $-\chi$ direction.

The remaining part,

$$
\mathscr{f}_{1 b}(\chi) \equiv-\frac{c}{2 e} \frac{\partial g_{0}}{\partial \theta} \frac{\partial g_{0}}{\partial \psi}+\frac{c}{\mathrm{eN}^{2}} \oint \mathrm{d} \chi^{\prime} \frac{\partial p_{\varphi}}{\partial \psi}\left(\chi^{\prime}\right) \int_{\chi}^{\chi^{\prime}} \mathrm{d} \chi^{\prime \prime} \frac{\partial \rho_{\varphi}}{\partial \theta}\left(\chi^{\prime \prime}\right)
$$

of Eq. (B2) is simplified first by integrating half of the second term by parts. The product of the integrals cancels the first term in Eq. (B8) and what remains is

$$
\delta_{1 b}(\chi) \equiv \frac{c}{e N^{2}}\left(\oint \mathrm{d} \chi^{\prime} \frac{\partial p_{\varphi}}{\partial \psi}\left(\chi^{\prime}\right) \int_{\chi}^{\chi^{\prime}} \mathrm{d} \chi^{\prime \prime} \frac{\partial p_{\varphi}}{\partial \theta}\left(\chi^{\prime \prime}\right)-\oint \mathrm{d} \chi^{\prime} \frac{\partial p_{\varphi}}{\partial \theta}\left(\chi^{\prime}\right) \int_{\chi}^{\chi^{\prime}} \mathrm{d} \chi^{\prime \prime} \frac{\partial p_{\varphi}}{\partial \psi}\left(\chi^{\prime \prime}\right)\right)
$$

In evaluating these intcgrals, it is necessary to remember that the integrations in Eq. (45) are along the contour in the direction of motion with $\chi^{\prime \prime}$ later in time than $\chi^{\prime}$.

To be more specific, we must now limit ourselves to the case of positive passing particles. In this case we evaluate one of these terms of Eq. (B9) using $[-\pi, \pi]$ as our basic interval. The maxima of this evaluation have the same value, because the integrations are carried out with $\theta$ held constant. The first integral in Eq. (B9) is 


$$
\begin{aligned}
\mathrm{Q} & \equiv \oint \mathrm{d} \chi^{\prime} \frac{\partial p_{\varphi}}{\partial \psi}\left(\chi^{\prime}\right) \int_{\chi}^{\chi^{\prime}} \mathrm{d} \chi^{\prime \prime} \frac{\partial p_{\varphi}}{\partial \theta}\left(\chi^{\prime \prime}\right) \\
& =\int_{-\pi}^{\chi} \mathrm{d} \chi^{\prime} \frac{\partial p_{\varphi}}{\partial \psi}\left(\chi^{\prime}\right) \int_{\chi}^{\chi^{\prime}+2 \pi} \mathrm{d} \chi^{\prime \prime} \frac{\partial p_{\varphi}}{\partial \theta}\left(\chi^{\prime \prime}\right)+\int_{\chi}^{\pi} \mathrm{d} \chi^{\prime} \frac{\partial p_{\varphi}}{\partial \psi}\left(\chi^{\prime}\right) \int_{\chi}^{\chi^{\prime}} \mathrm{d} \chi^{\prime \prime} \frac{\partial p_{\varphi}}{\partial \theta}\left(\chi^{\prime \prime}\right) .
\end{aligned}
$$

In the first term, where $\chi^{\prime}$ is less than $\chi, 2 \pi$ must be added to the upper limit of the first inner integral so that it is after $\chi$. In the negative passing case, $2 \pi$ would have to be subtracted from the upper limit of the second inner integral.

Having taken care of this requirement, the integrals may now be manipulated by the usual techniques. Thus,

$$
\mathrm{Q}=\frac{\partial g_{0+}}{\partial \psi} \frac{\partial g_{0_{+}}}{\partial \theta}+\int_{-\pi}^{\pi} \mathrm{d} \chi^{\prime} \frac{\partial p_{\varphi}}{\partial \psi}\left(\chi^{\prime}\right)\left(\int_{\chi}^{0} \mathrm{~d} \chi^{\prime \prime} \frac{\partial p_{q_{q}}}{\partial \theta}\left(\chi^{\prime \prime}\right)+\int_{0}^{\chi^{\prime}} \mathrm{d} \chi^{\prime \prime} \frac{\partial p_{\varphi_{q}}}{\partial \theta}\left(\chi^{\prime \prime}\right)\right)
$$

The last term in the integrand vanishes upon integration, because $p_{\varphi}$ and its $\theta$ and $\psi$ derivatives (at constant $\chi$ ) are even in $\chi$ about the origin. Hence, the integral vanishes because it is of an even function multiplied by the integral from the origin of an even function, which produces an odd function. The other term in the integral factors, because the interior integral is independent of the argument of the exterior integral. As a result,

$$
\mathbf{Q}=\frac{\partial g_{0_{t}}}{\partial \psi} \frac{\partial g_{0_{t}}}{\partial \theta} \cdot \frac{\partial g_{0_{t}}}{\partial \psi}\left(\chi^{\prime}\right) \int_{0}^{\chi} \mathrm{d} \chi^{\prime} \frac{\partial p_{\theta}}{\partial \theta}\left(\chi^{\prime}\right)
$$

The remaining integral in Eq. (B9) is analyzed identically. The same result is obtained, but with a minus sign and the derivatives with respect to $\psi$ and $\theta$ interchanged. The net result is then 


$$
g_{+1 b}(\chi) \equiv \frac{c}{e N^{2}}\left[g+0, \int_{0}^{\chi} \mathrm{d} \chi^{\prime} p_{\varphi}\left(\chi^{\prime}\right)\right]
$$

Combining Eqs. (B6) and (B/3) gives the total first-order correction. A similare analysis gives the total first-order correction for the negative passing particles. These two results may be combined as

$$
\begin{aligned}
g_{ \pm 1}= & \frac{l t}{\mathrm{~N}} g_{0 u}+\frac{l \mathrm{mcuB}_{\varphi}}{\mathrm{eBN}} \frac{\partial g_{+0}}{\partial \psi} \cdot \frac{l \mathrm{~m}^{2} \mathrm{c}}{\mathrm{eN}^{2}} \frac{\partial}{\partial \psi} \int_{0}^{\pi} \mathrm{d} \chi^{\prime}\left(\mathrm{uB}_{\varphi} / \mathrm{B}\right)^{2} \\
& \pm \frac{\mathrm{c}}{\mathrm{eN^{2 }}}\left[g_{+0} \cdot \int_{0}^{\chi} \mathrm{d} \chi^{\prime} \mathrm{p}_{\varphi}\left(\chi^{\prime}\right)\right],
\end{aligned}
$$

in a form without double integrals. The primary difference for the trapped particles is that the shift involved in going from Eq. (B9) to (B10) is one around the closed trapped particle contour, rather than a shift of $2 \pi$ in the angle. A secondary difference is that there is no integral of $u^{2}$ as noted above. The result is

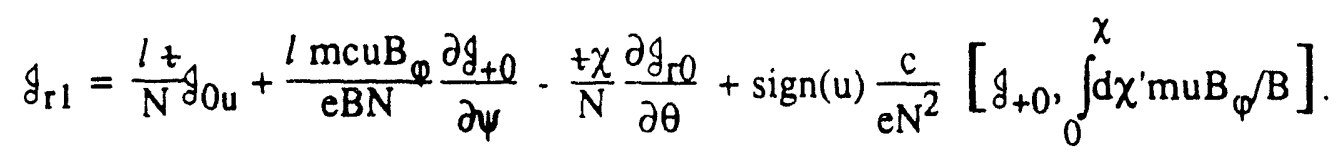

\section{B 2. Expression in terms of special functions}

For numerical evaluation, it is useful to put these expressions for the adiabatic invariant corrections in terms of special functions, for which routines are in existence. There are just two integrals that have not yet been put in such terms, the integral appearing in the third term of Eq, (B14) and the integral on the left of the bracket in Eqs. (B14) and (B15). 
The first of the just mentioned terms is the definite integral of a rational function of $\cos (\chi)$. Such integrals may be evaluated using complex variable techniques, or by using Eq. 2.554 \# 1 of Ref. 23. These methods give

$$
\int_{0}^{\pi} \mathrm{d} \chi^{\prime}(u / B)^{2}=\frac{\pi \mu}{2 \varepsilon_{r} B_{0}} \frac{\sigma^{3} \eta+2 \sigma^{2} \eta \cdot \sigma^{2}}{(1+\sigma / 4)^{3 / 2}}
$$

The second of the aforementioned integrals can be expressed in terms of incomplete elliptic integrals. Standard manipulations give

$$
\int_{0}^{\chi} \mathrm{d} \chi^{\prime} \mathrm{mu} / \mathrm{B}=\frac{4 \sqrt{\varepsilon_{\mathrm{r}} m \mu / \mathrm{B}_{0}}}{1-\varepsilon_{\mathrm{t}} \cos (\theta)-\varepsilon_{\mathrm{r}}}[(1+\sigma \eta) \Pi(\sigma, 1 / \eta, \chi)-F(1 / \eta, \chi)] / \sigma \sqrt{\eta} .
$$

where

$$
\Pi(\sigma, 1 / \eta, \chi)=\int_{0}^{\chi} \frac{\mathrm{d} \chi^{\prime}}{\left(1+\sigma \sin ^{2}\left(\chi^{\prime}\right)\right) \sqrt{1-\sin ^{2}\left(\chi^{\prime}\right) / \eta}}
$$

is the incomplete elliptic integral of the thiro kind and

$$
F(1 / \eta, \chi)=\int_{0}^{\chi} \frac{d \chi^{\prime}}{\sqrt{1-\sin ^{2}\left(\chi^{\prime}\right) / \eta}}
$$

is the incomplete elliptic integral of the first kind.

We do not present the full combined expression for the adiabatic corrections because the expression in this form was never obtained. Instead, the various derivatives were calculated in terms of special functions and the results were summed numerically. 


\section{References}

1 L. M. Kovrizhnykh and S. G. Shasharina, Nucl. Fusion 36, 453 (1990)

2 L. M. Kovrizhnykh, and S. G. Shasharina, Comments on Plasma Phys. and Controlled Fusion XII, 305 (1989).

3 H. E. Mynick, Phys. Fluids 26, 2609 (1983).

4 H. E. Mynick and W. N. G. Hitchon, Nucl. Fusion 24, 317 (1984).

5 D. Dobrott and J. M. Greene, Phys. Fluids 8, 223 (1988).

6 P. N. Yushmanov, in Reviews of Plasma Physics, B. B. Kadomtsev, ed., 16, 102 (1987).

7 R. J. Goldston and H. H. Towner, J. Plasma Phys. 26, 283 (1981).

8 V. P. Nagornij and V. A. Yavorskij, Sov. J. Plasma Phys. 15, 309 (1989).

9 S. P. Hirshman, K. C. Shaing, W. I. van Rij, C. O. Beasley, Jr., and E. C. Crume, Jr., Phys. Fluids 29, 2851 (1986).

10 J. R. Cary, C. L. Hedrick, and J. S. Tolliver, Phys. Fluids 31, 1586 (1988).

11 M. Kruskal, J. Math. Phys. 3, 806 (1962).

12 A. H. Boozer, Phys. Fluids 24, 1999 (1981).

13 J. D. Meiss and R. D. Hazeltine, Phys. Fluidis (1991).

14 A. H. Boozer, Phys. Fluids 23, 905 (1980).

15 J. Buchner and L. M. Zelenyi, J. Geophys. Res. 94, 11821 (1989).

16 J. R. Cary, D. F. Escande, and J. L. Tennyson, Phys. Rev. A 34, 4256 (1986); A. I. Neishtadt, Sov. J. Plasma Phys. 12, 568 (1986).

17 J. R. Cary, and R. T. Skodje, Physica D 346, 287 (1989).

18 C. R. Menyuk, Phys. Rev. A31, 3282 (1985).

19 J. R. Cary, Bull. Am. Phys. Soc. 30, 1579 (1985). 
20 V. I. Arnold, Mathematical Methods of Classical Mechanics (Springer-Verlag, 1978); L. Michelotti, in AIP Conference Proceedings \#184: Physics of Particle Accelerators (American Institute of Physics, New York, 1989) Vol 1, p. 892.

21 R. G. Littlejohn, J. Plasma Phys. 29, 111 (1983).

22 J. F. Lyon, B. A. Carreras, K. '.. Chipley, M. J. Cole, J. H. Harris, T. C. Jernigan, R. L. Johnson, V. E. Lynch, B. E. Nelson, J. A. Rome, J. Sheffield, and P. B. Thompson, Fusion Technology 10, 179 (1986).

23 I. S. Gradshteyn and I. M. Ryzhik, Table of Integrals, Series, and Products, (Academic Press, New York, 1980). 
Figure Captions

Fig. 1. Potential energy $(\mu B+e \Phi)$ along a field line in an asymmetric corus. Passing particles have sufficient energy to pass over all the local potential energy maxima. Locally trapped particles are trapped between two successive maxima. Toroidally trapped particles have sufficient energy to pass over some maxima but not all. Hence, toroidally trapped particles are locally passing.

Fig. 2. Trapping of a trajectory due to the change of the potential energy due to drift to neighboring field lines. When the particle crosses the local maximum moving to the right the potential energy has the solid profile. After reflection (heavier portion of horizontal line) the particle has drifted to a field line with potential energy profile shown as a dashed curve.

Fig. 3. Contours of the adiabatic invariants of Ref. 10 for $N=12, \gamma_{\mu}=1.81 \times 10^{-4}$, $\gamma_{E}=2 \times 10^{-4}$. (These parameters are explained in Sec. V.) The contours for the different states meet at the transition curve, the heavy partial circle. Positive passing contours are solid, negative passing contours are long dashed, and trapped contours are short dashed. Trajectories move along the contours in the direction of the arrow. Revolving these contours in the toroidal angle gives the patched tori.

Fig. 4. Energy diagram for the trapping process. Trajectory a asymptotes to the right maximum. Trajectory $b$ asymptotes to the left maximum after reflecting off the right maximum. Trajectory $c$ asymptotes directly to the left maximum.

Fiss. 5. Phase space representation of the processes shown in Fig. 4. The incoming asymptotes are dashed while the instantaneous contours of the Hamiltonian are solid.

Fig. 6. Transition processes in a torus for fixed energy and initial value of the adiabatic invariant. The line of magnetic maxima are drawn as heavy lines. The trajectory passing 
just above $c$ is reflected by the local maximum at $c$. The trajectory passing just below $c$ just barely passes over this maximum, but due to drifts is unable to pass over the maximum after reflection and so becomes trap, ed. The point $b$ separates those trajectories (above $b$ ) just trapped by the maximum at $b$ from those (below $b$ ) that do pass over this local maximum and so are reflected. The point $c^{\prime}$ is identical to $c$ except for the displacement of one field period.

Fig. 7. Parameters of the magnetic variation along a field line in the neighborhood of a single well.

Fig. 8. The numerical trapping probability versus the poloidal angle of trapping, $\theta$, along the transition circle for $N=12, \gamma_{\mu}=1.81 \times 10^{-4}, \gamma_{E}=2 \times 10^{-4}, \gamma_{\Phi}=0$ for two cases: $P_{0}$ - when the adiabatic invariant is kept constant through the zeroth order, and $P_{1}$ - through the first order.

Fig. 9. The trapping probability $P$ versus the poloidal angle of trapping, $\theta$, along the transition circle for $N=12, \gamma_{\mu}=1.81 \times 10^{-4}, \gamma_{E}=2 \times 10^{-4}, \gamma_{\Phi}=0$. Dots show the numerical results. Solid curves are analytical results of Ref. 1 (labeled KS), Ref. 7 (labeled GT), Ref. 8 (labeled NY), Ref. 10 (labeled CHT) and the present work (labeled CS).

Fig. 10. The trapping probability $P$ versus the poloidal angle of trapping, $\theta$, along the transition circle for $N=12, \gamma_{\mu}=1.81 \times 10^{-4}, \gamma_{E}=3 \times 10^{-4}$, and $\gamma_{\Phi}=2 \times 10^{-4}$. Dots show the numerical results. The analytical results of Refs. 1, 7,8,10 and the present work are the solid curves labeled as in Fig. 9.

Fig. 11. The trapping probability $P$ versus the poloidal angle of trapping, $\theta$, along the transition circle for $N=12, \gamma_{\mu}=1.81 \times 10^{-4}, \gamma_{E}=1 \times 10^{-4}$, and $\gamma_{\Phi}=-2 \times 10^{-4}$. Dots show 
the numerical results. The analytical results of Refs. $1,7,8,10$ and the present work are the solid curves labeled as in Fig. 9.

Fig. 12. As shown in going from panel (a) to panel (b), a slight shift of the inner partial circle, the adiabatic invariant contour for locally trapped particles in a machine with negative electrostatic potential, causes the particles to flow into the transition boundary at the top rather than the bottom.

Fig.13. The trapping probability $\mathrm{P}$ versus the poloidal angle of trapping, $\theta$, along the transition line for the tokamak case for $\gamma_{\mu}=0.93 \times 10^{-4}, \gamma_{E}=1 \times 10^{-4}$, and $\gamma_{\Phi}=0$. Dots show the numerical results. The analytical results of Ref. 7 and the present work are the solid curves labeled as in Fig. 9. 


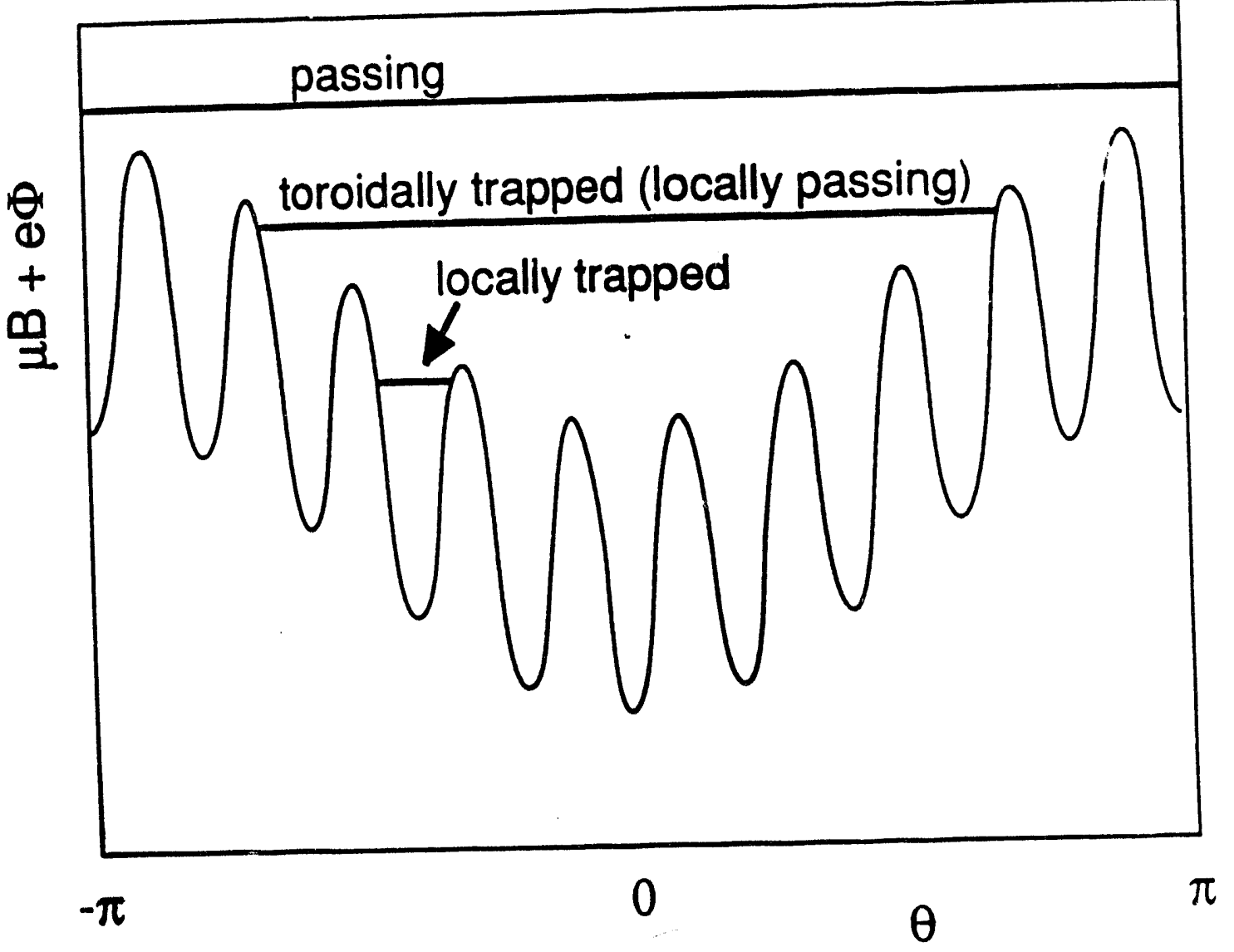

Fig. 1 


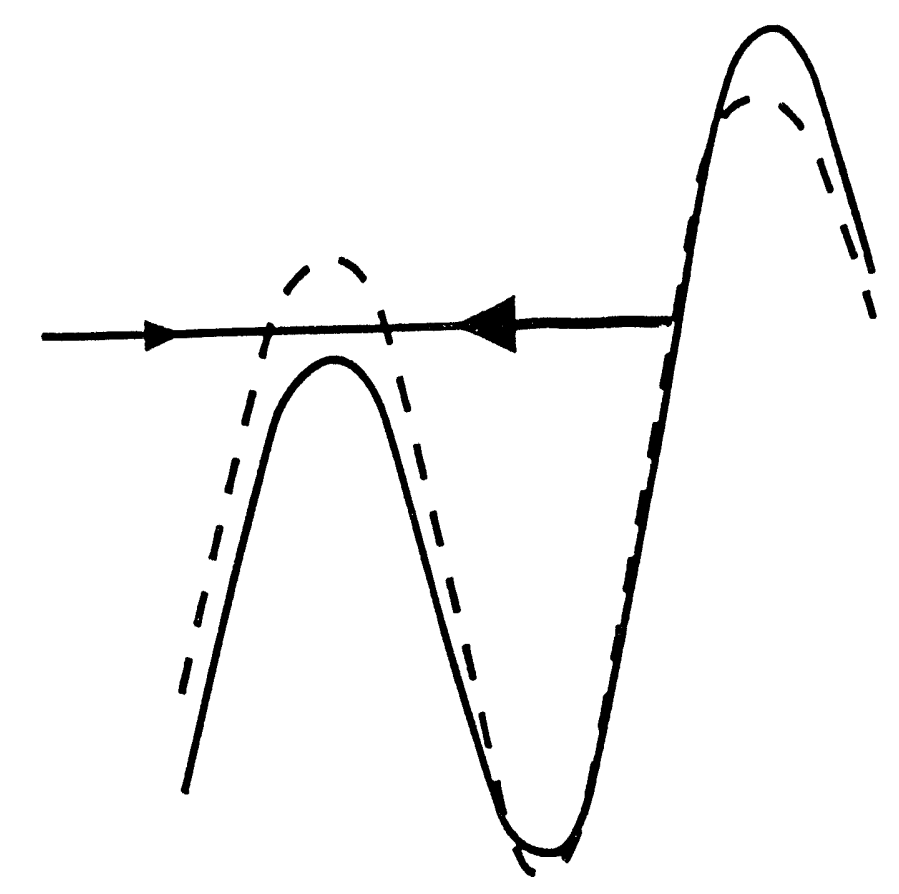

Fig. 2 


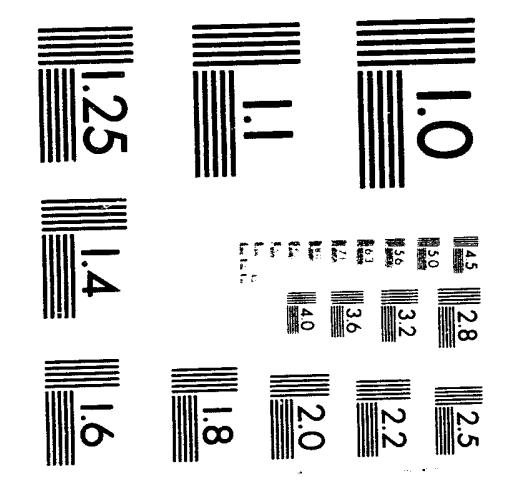



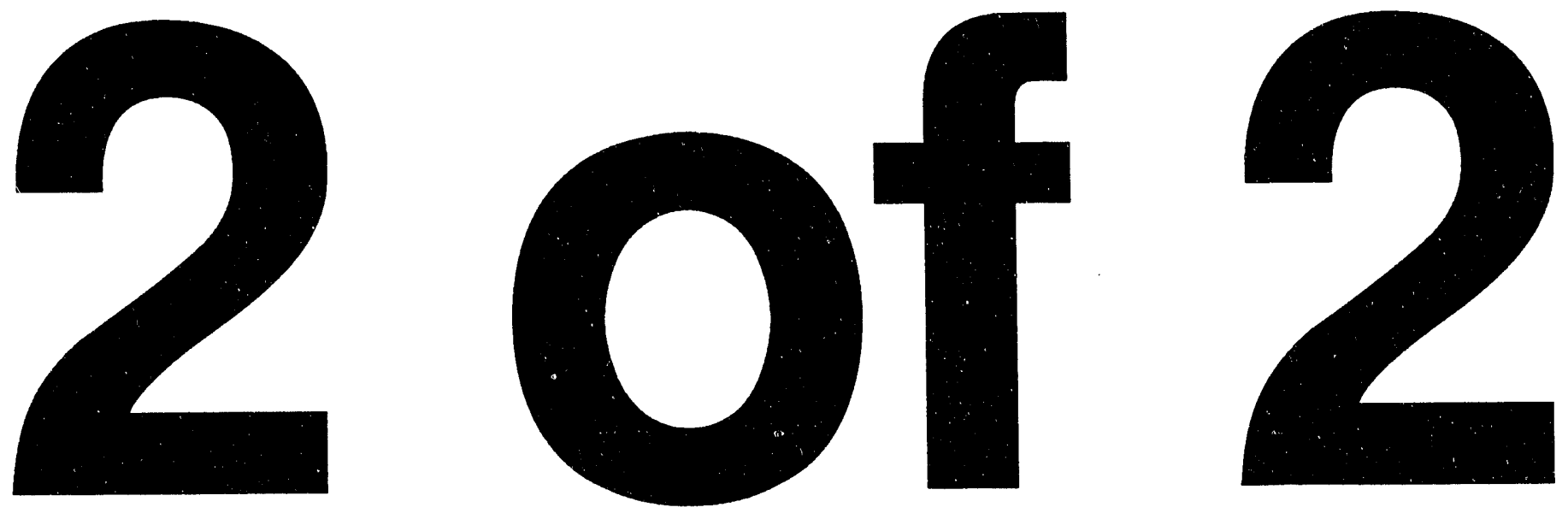



$$
\text { XIf }
$$




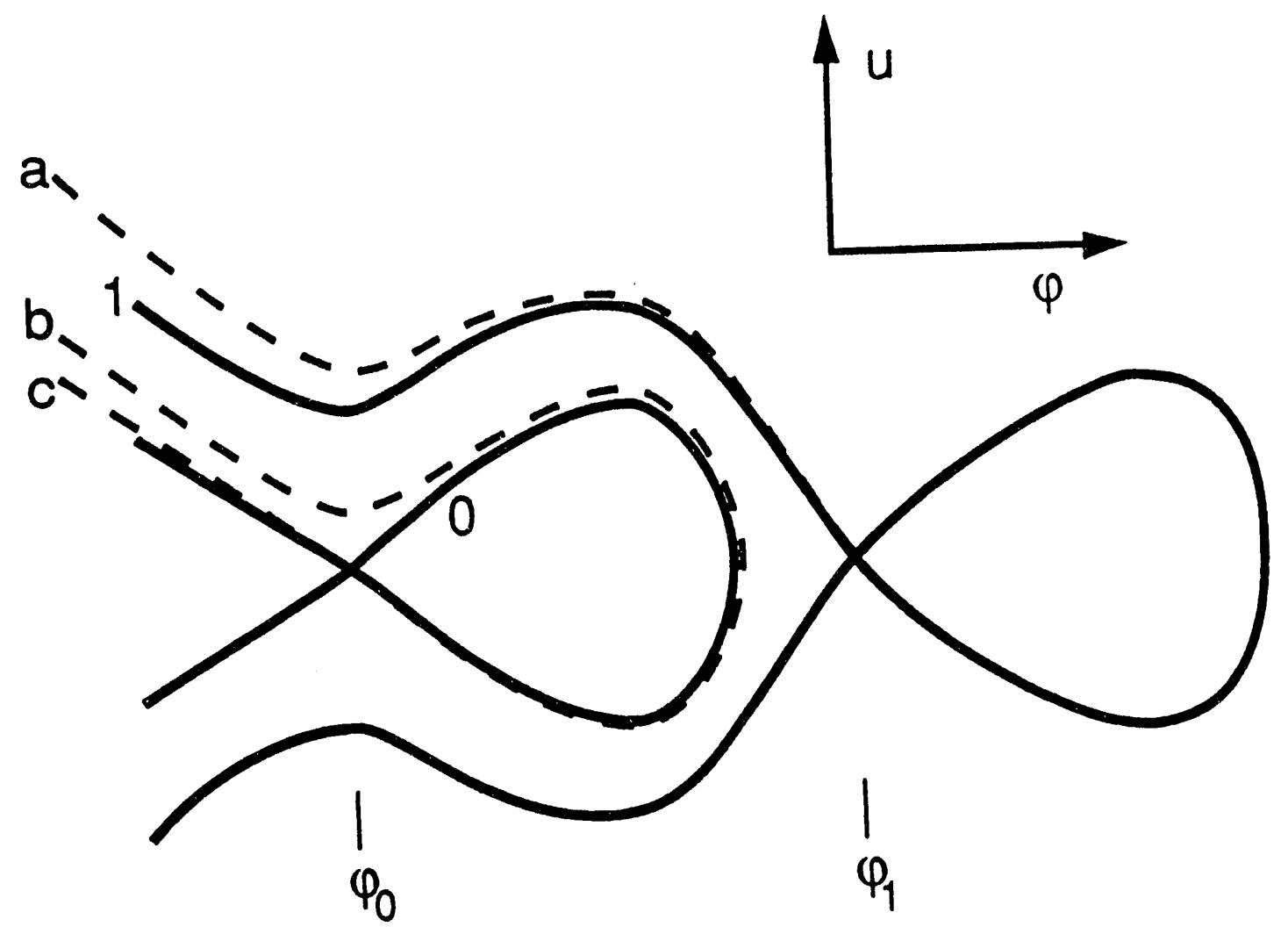

Fig. 5 


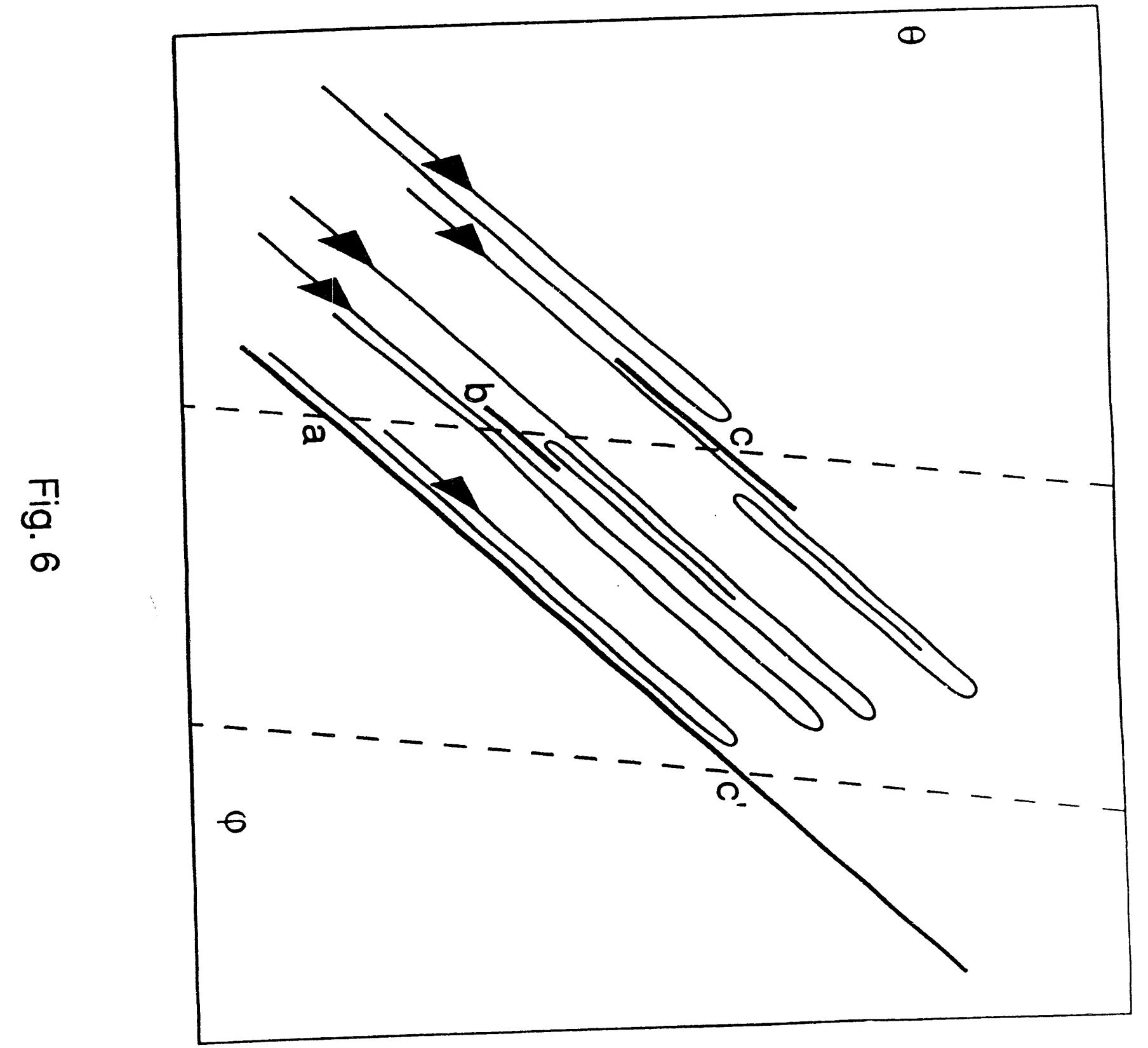

• 
$\frac{\pi}{\overline{0}}$

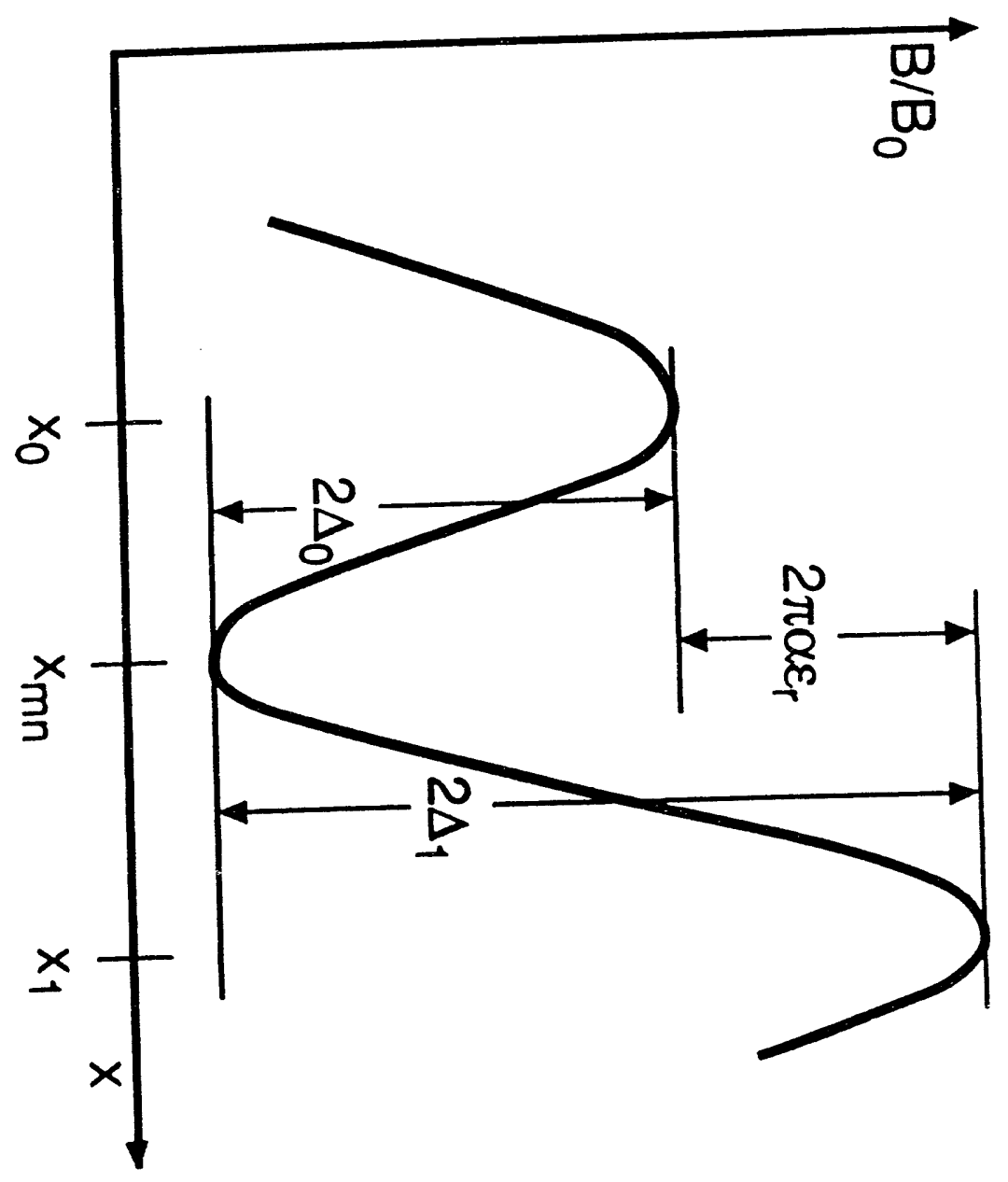

• 


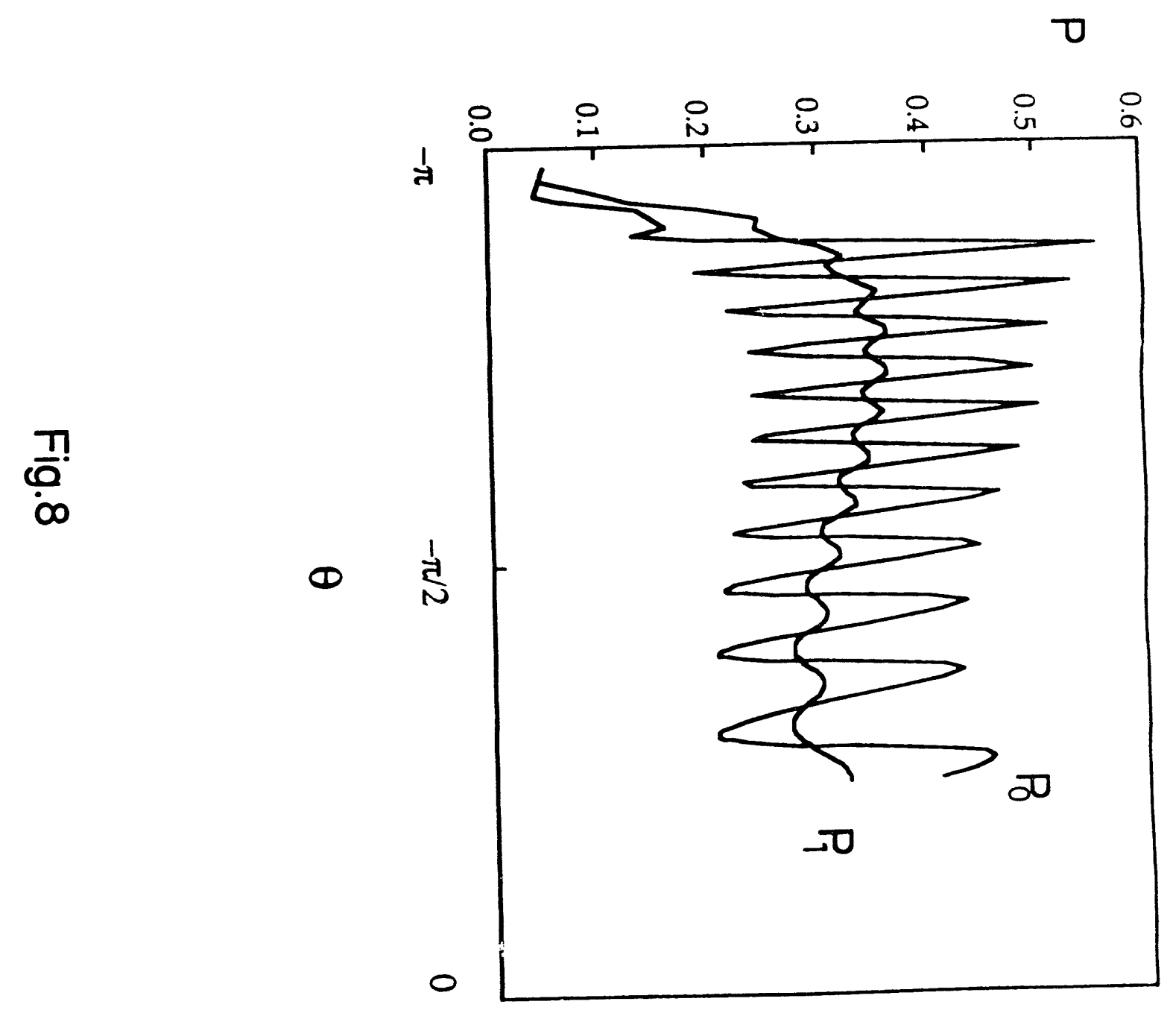




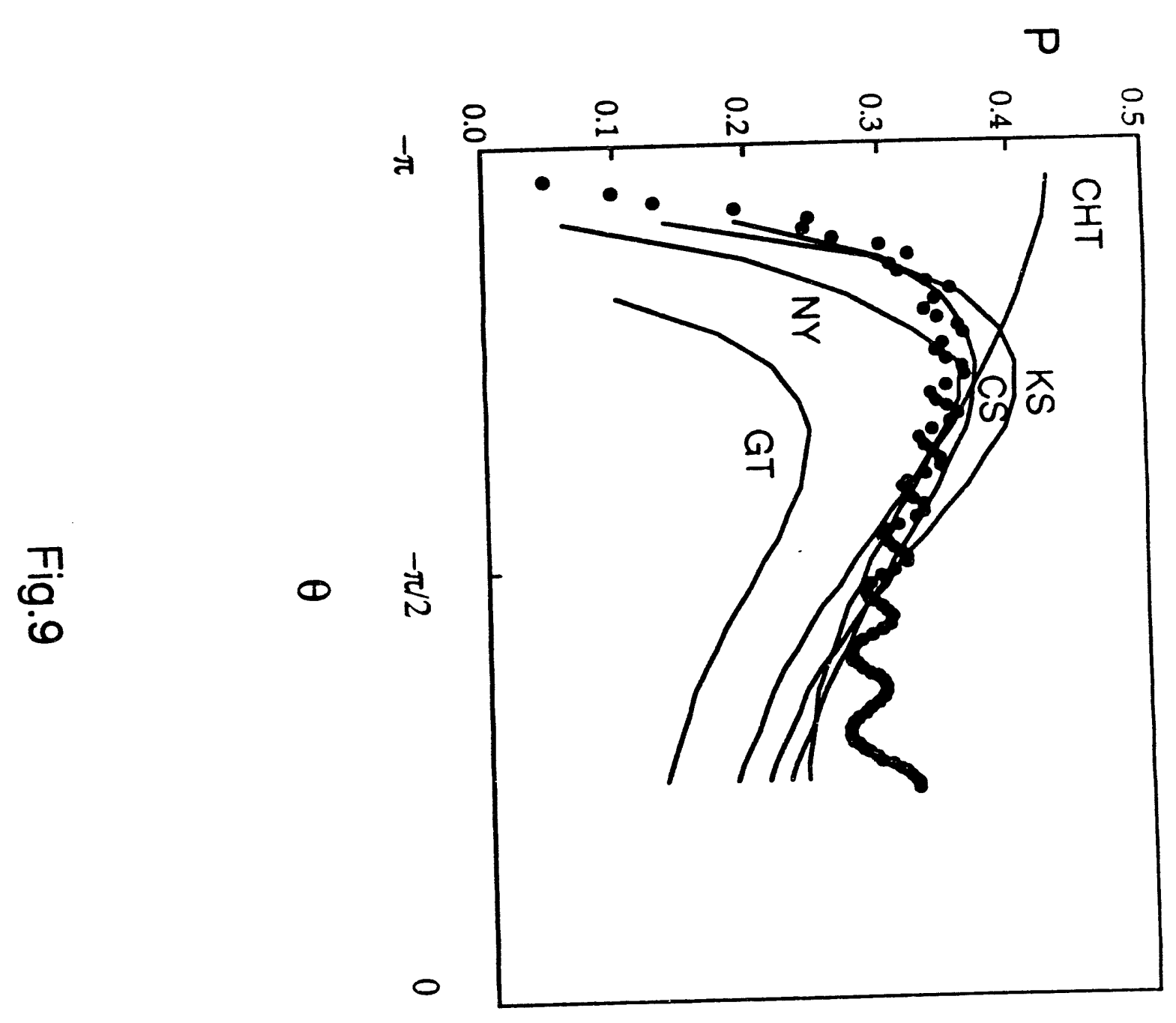

• 


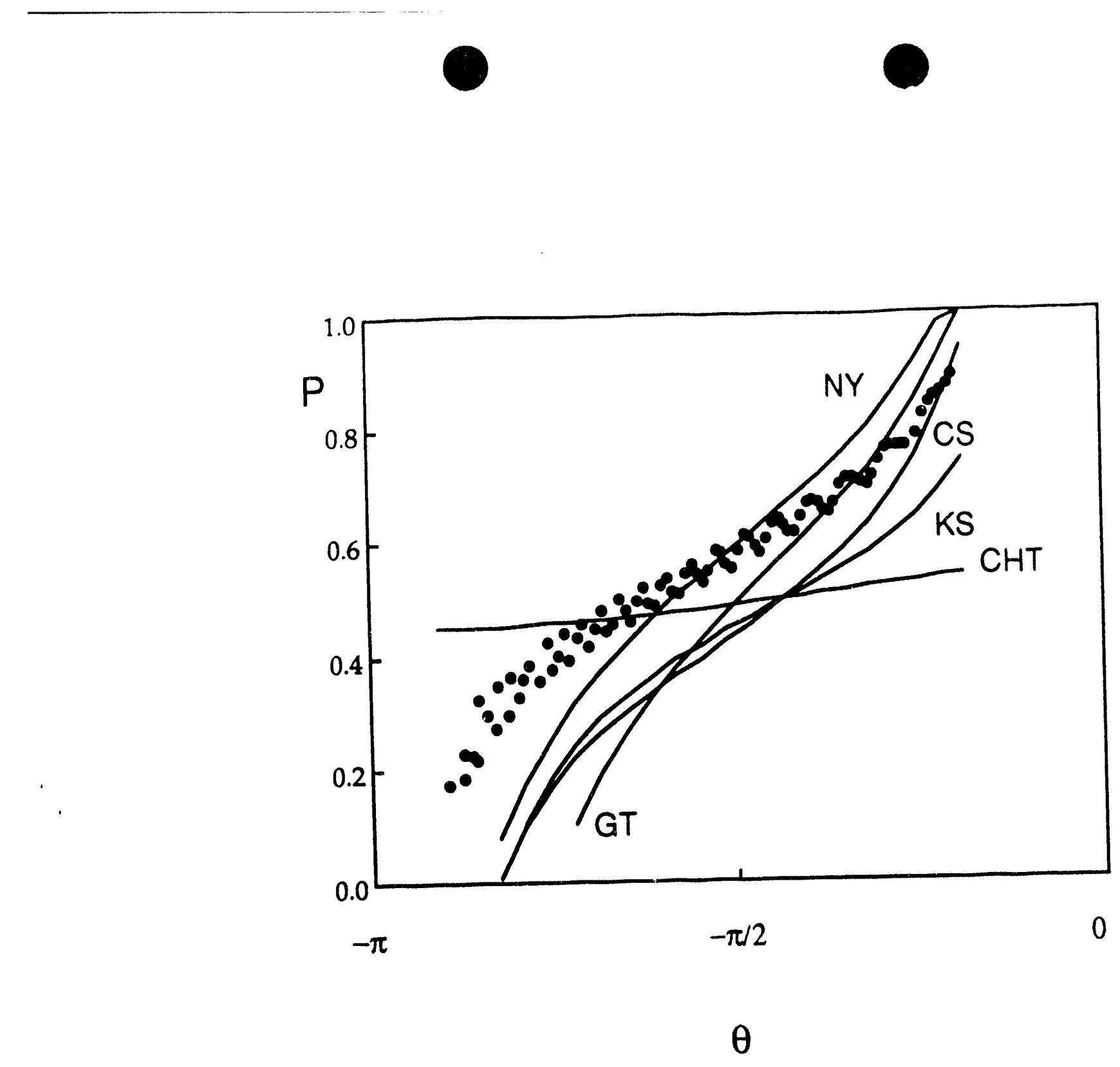

Fig.10 
$\stackrel{9}{9}$

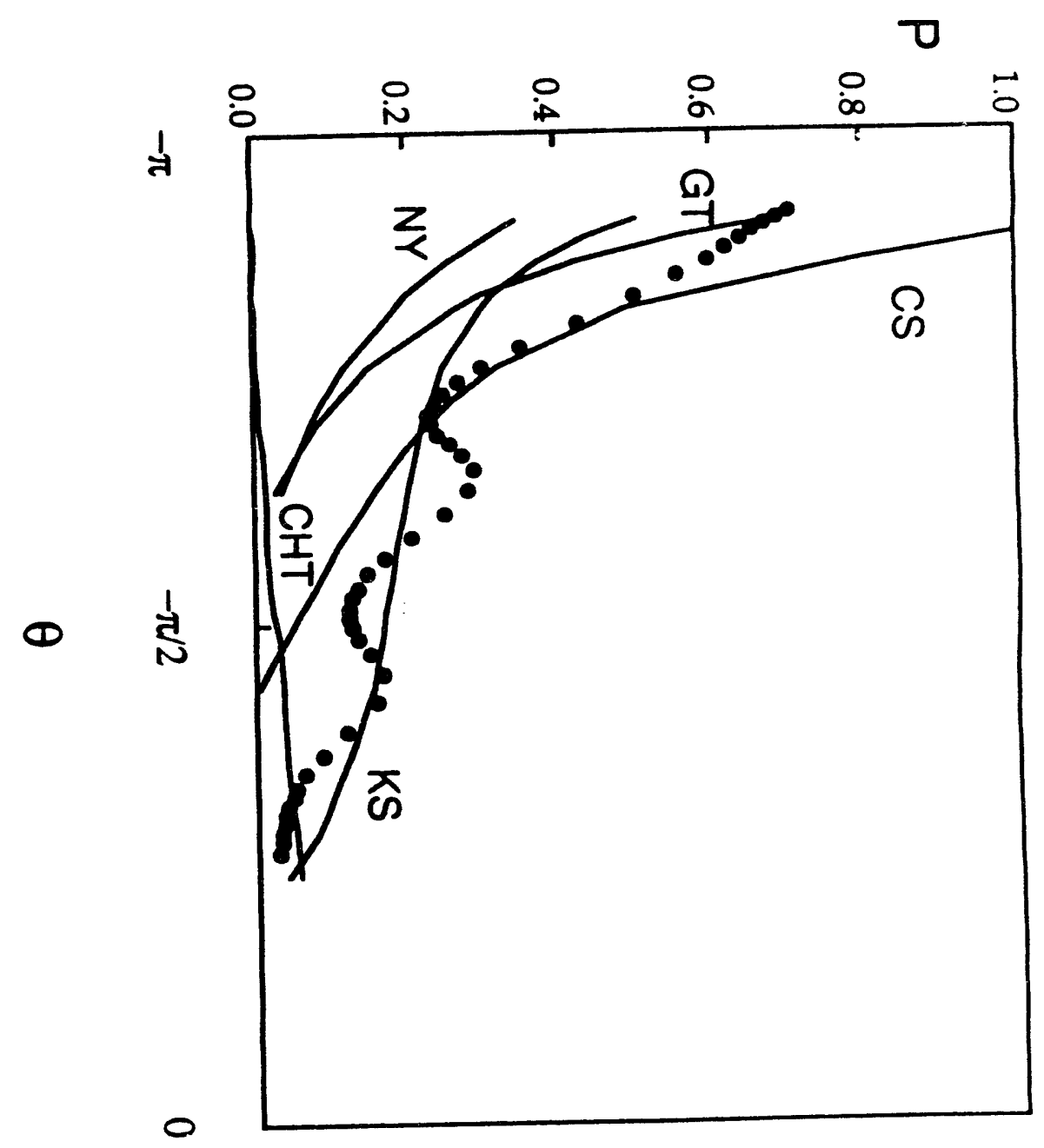




$$
8
$$




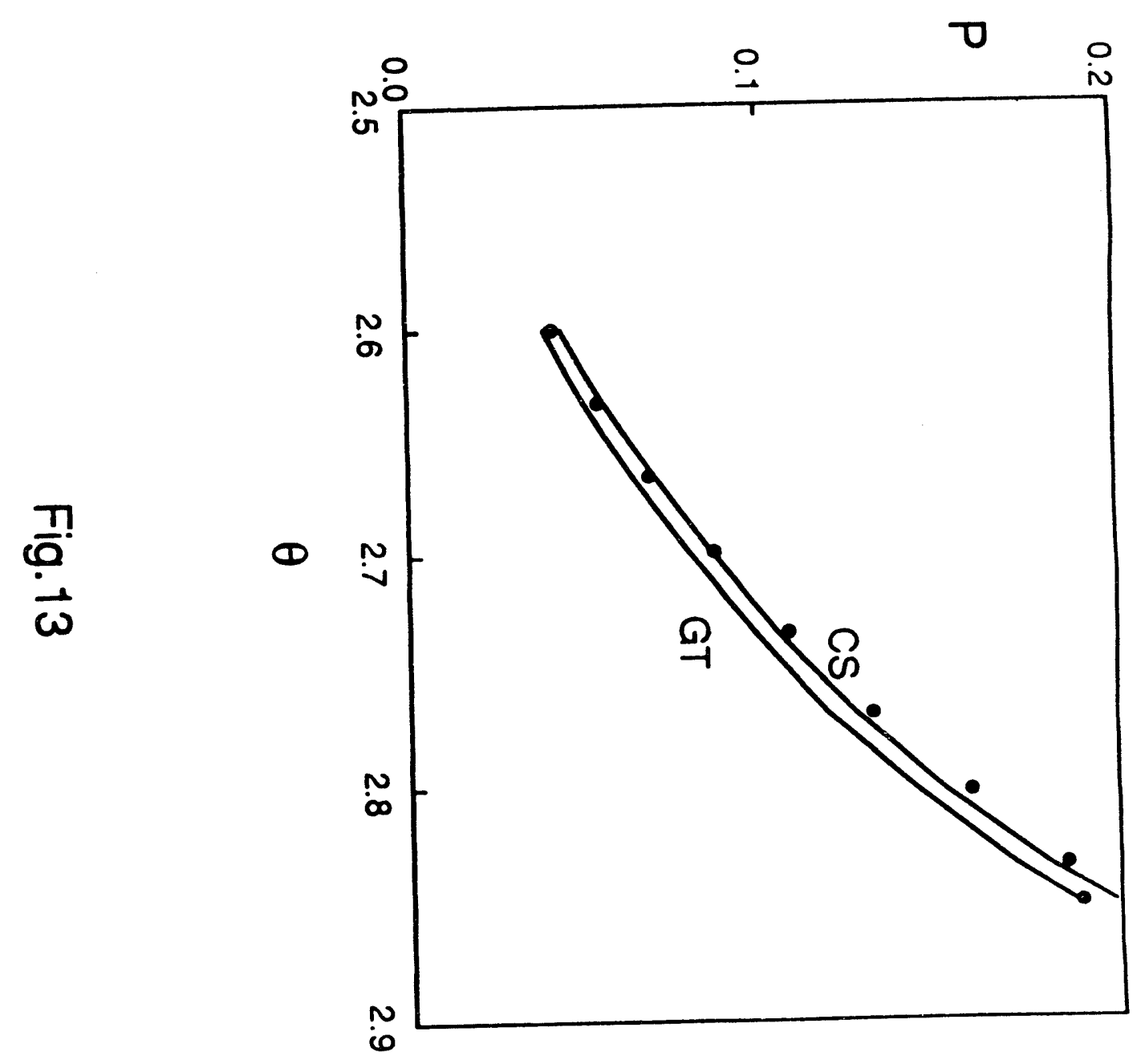


Appendix B. Ripple Induced Stochastic Loss of Alpha Particles in a Tokamak 
Ripple Induced Stochastic Loss of Alpha Particles in a Tokamak

\author{
S. G. Shasharina, ${ }^{*}$ John R. Cary
}

Department of Astrophysical, Planetary and Atmospheric Sciences, University of Colorado, Boulder CO-80309-0391

We solve the Fokker-Planck equation for alpha particles in a tokamak including both pitch angle scattering and slowing down. Losses of the toroidally trapped particles due to stochastic diffusion related to the magnetic ripple are accounted for by assuming that all the stochastic orbits immediately leave the device. This assumption permits us to solve the problem by introducing the loss cone corresponding to the stochastic region of the velocity space. The eigenvalue problem is solved numerically to show that the additional stochastic loss of energy due to the scattering into the loss cone substitute about $30 \%$ of the birth energy for typical fusion parameters.

* Permanent address: General Physics Institute of Ac. Sci. of Russia, Moscow, 117942, Russia, CIS. 
1.

Introduction

Roughly $20 \%$ of the energy of fusion reactions in a D-T plasma is put into energetic alpha particles [1]. The confinement of these alphas is critical to the development of fusion reactors. Nevertheless, even the classical transport problems - not involving MHD phenomena - are not studied sufficiently well. The complexity of this study is due to the fact that too many different phenomena are responsible for the alphas losses $[1,2]$.

In fact, alpha confinement is governed by both collisionless and collisional mechanisms. Alphas born with chaotic orbits $[2,3]$ are lost very quickly - on a time scale shorter than any of the collisional transport rates $[2,4]$. These chaotic orbits, which arise due to the magnetic ripple or magnetic turbulence, are characterized by certain values of the energy and pitch angle. The pitch angle and energy change due to collisional mechanisms. Alphas slow down due to friction on the background electrons. The pitch angle of an alpha changes due to scattering off the background ions. These collisions can put an alpha on a chaotic orbit, so it is immediately lost. Thus, a calculation of the alpha density and the energy deposition must include both these collisional and chaotic mechanisms.

To this end several Monte Carlo codes have been developed. The most basic of these [5-7] follow the guiding center trajectories of many particles perturbed by collisions. Other codes $[8,9]$ use mapping methods, which reduce the required time by an order of magnitude (at some cost of accuracy) by replacing the integration over a bounce time by a mapping representing the bounce period.

Unfortunately, such Monte Carlo codes have the disadvantage of requiring large amounts of computer time. For this reason the RIPLOS code [2] was developed to determine the rate of rapid alpha loss due to chaotic mechanisms. This code is much 
faster than the previously mentioned ones, because the simple criterion for chaotic motion is used rather than integration of the guiding center trajectories. The particles satisfying this criterion are assumed to be lost immediately, while those not satisfying this criterion are assumed to deposit their energy in the plasma.

On the other hand, there was an attempt to take into account both mechanisms analytically [10] with stochastic diffusion accounted for by assuming that all toroidally trapped particles are stochastic. A serious simplification (and, simultaneously, disadvantage) of this calculation is the use of a Bhatnagar-Gross-Krook operator, which can not reproduce diffusive pitch angle scattering.

The goal of the present paper is to take these developments one step further to calculate the combined effects of the collisions and stochastic behavior. The FokkerPlanck equation for the distribution function is solved by ordering [11-13] in the ratio of the collision frequency to the bounce frequency. The effects of the collisionless (chaotic) loss are included by imposing the boundary condition that the distribution function vanish on the chaotic orbits. In essence, we solve the kinetic equation in the presence of the loss cone. Note, that the equations used, as well as the main results, are valid not only for alpha particles, but also for fast ions with an arbitrary distribution in the pitch angle.

The abundance of the literature on the alpha particle issue spares us from showing all the details of choosing the particular form of the kinetic equation. We also omit the description of the stochastic diffusion mechanism, since we need only the specification of the stochastic region of the velocity space.

In Sec. 2 we present the formulation of the problem, which is to find the distribution function of fast ions in a tokamak, when the effects of slowing-down and pitch-angle scattering are taken into account. In Sec.3, we discuss the method of solving the problem formulated in Sec.2. This method implies an assumption of small radial displacements of 
the trajectories and low collision frequency. The separation of variables, analogous to that presented in $[12-14]$ is performed, which allows to us to solve the arising eigenvalue problem numerically. Sec. 4 presents the results of our calculation in terms of the relative flux of alphas into the stochastic region.

2. The problem: distribution function of fast ions in the presence of stochastic diffusion

\subsection{Kinetic equation for the axisymmetric torus}

We use the standard quasi-toroidal system of coordinates, $(r, \theta, \phi)$, with $r$ the minor radius, $\theta$ and $\phi$ the poloidal and toroidal angles, respectively. To the first order approximation of the guiding center theory, the stationary drift kinetic equation $[11,12]$ for the fast ions in axisymmetric tokamak can be written in the following form :

$$
\frac{\mathrm{u} \varepsilon}{\mathrm{qr}} \frac{\partial \mathrm{f}}{\partial \theta}=\mathrm{C}(\mathrm{f})+\mathrm{S}_{0} \delta\left(v-\mathrm{v}_{0}\right)
$$

Here, $f$ is the distribution function of the fast ions, $\frac{u \varepsilon}{q R}$ is the poloidal component of the parallel velocity, $u= \pm \sqrt{2(E-\mu B)}, q=1 / t$ is the safety factor, $R$ is the major radius, $R=R_{0}(1+\varepsilon \cos (\theta)), \varepsilon=r / R_{0}$ is the toroidicity, $C(f)$ is the collision operator, $S_{0}(\mu) \delta\left(v-v_{0}\right)$ is the source of the fast ions, born with velocity vo. The quantity $S_{0}$ can depend on the pitch angle which allows us to study the neutral beam ions also.

Everywhere, we imply that variables without indices correspond to $\alpha$-s or other fast ions under consideration.

The axisymmetric magnetic field has the form,

$$
B=B_{0} \frac{R}{R_{0}} \cong B_{0}(1-\varepsilon \cos (\theta)) \text {. }
$$


Here and elsewhere, we keep only the first order corrections in $\varepsilon$.

$$
\begin{aligned}
& \text { For } v_{i}<v<v_{e}, \text { the collision operator }[4-7,12,13] \text {, } \\
& C(f)=C_{p}(f)+C_{v}(f)
\end{aligned}
$$

consists of the pitch-angle scattering and slowing-down operators,

$$
\begin{aligned}
& C_{p}(f)=2 z v\left(\frac{v_{c}}{v}\right)^{3} \frac{u}{B} \frac{\partial}{\partial \mu} \mu u \frac{\partial f}{\partial \mu}, \\
& C_{v}(f)=\frac{v}{v^{2}}\left(v^{3}+v_{c}^{3}\right) f
\end{aligned}
$$

Here, $z=\frac{Z_{e f f} m_{i}}{2 m}, v_{c}^{\prime}=\left(\frac{3 m_{e} \sqrt{\pi}}{4 m_{i}}\right)^{1 / 3} v_{e}, v$ is the collision frequency which is independent of the energy.

For further convenience we introduce a new pitch angle variable (see [12]),

$$
\xi=\sqrt{1-\frac{2 \mu B_{0}}{v^{2}}}
$$

use of which reduces Eqs.(1) to

$$
\frac{u \varepsilon}{q r} \frac{\partial f}{\partial \theta}=z v\left(\frac{v_{c}}{v}\right)^{3} \frac{u}{\xi v} \frac{\partial}{\partial \xi} \frac{\left(1-\xi^{2}\right) u}{\xi v} \frac{\partial f}{\partial \xi}+\frac{v}{v^{2}} \frac{\partial}{\partial v}\left(v^{3}+v_{c}^{3}\right) f+S_{0} \delta\left(v-v_{0}\right)
$$

Eq.(7) is the main subject of investigation in all studies of fast ion transport in a tokamak.

\subsection{Ripple effects}

A real tokamak is not axisymmetric, its initial axisymmetry being destroyed by the discrete set of coils, which create the toroidal magnetic field. Magnetic ripple leads to stochastic diffusion [2] resulting in rapid loss of the toroidally trapped alphas. This mechanism of particle loss is very fast. That is why we can assume that the stochastic 
particles leave the system immediately $[2,4,10]$. The region of stochasticity is easily specified in $v$ and $\xi$ coordinates: for the particle to become stochastic, the following inequalities should be fulfilled

$$
\begin{aligned}
& \xi<\xi_{1}, \\
& v \geq v_{s},
\end{aligned}
$$

with the border values,

$$
\begin{aligned}
& \xi_{\mathrm{t}}=\sqrt{\frac{\varepsilon}{1+\varepsilon}}, \\
& \mathrm{v}_{\mathrm{s}}=\frac{\Omega}{2 \delta}\left(\frac{\varepsilon}{\pi \mathrm{Nq}}\right)^{3 / 2} \frac{1}{\mathrm{dq} / \mathrm{dr}}, \quad \Omega=\frac{\mathrm{eB}_{0}}{\mathrm{mc}} .
\end{aligned}
$$

Condition (8) means that, to be stochastic, the particle should be toroidally trapped, while Eq.(9) represents the Goldston-White-Boozer criterion of stochastization [3], rewritten rather in terms of the critical velocity rather than in terms of the critical magnitude $\delta_{c}$ of ripple. In what follows, we neglect the dependence of $\varepsilon$ and $v_{S}$ on the poloidal angle, $\theta$, thinking of $\varepsilon$ as a maximum on the magnetic surface. Then taking this dependence into account can only reduce the value of the losses obtained below.

The ripple perturbation leads to fast loss of the particles in the region specified by Eqs.(8-11) which can be thought of as a loss cone. With all that taken into account, we reduce the study of the stochastic loss of the fast ions to the solution of the axisymmetric kinetic equation (7) with B given by Eq.(2) in the region $M$ (see Fig.1)

$$
M \equiv\left\{v \geq v_{s}, \xi_{1} \leq \xi \leq 1\right\},
$$

with the following boundary condition

$$
f\left(\xi_{1}\right)=0, v>v_{s}
$$

The distribution function found from Eqs.(7),(13) describes the fast ion distribution with the loss cone in the stochastic velocity space region. To evaluate the 
stochastic loss, we do not need the solution for $v<v_{s}$, where, by the way, we can not ignore the energy diffusion in comparison with the drag term.

\section{Separation of variables and the eigenfunction problem}

Since the high energy ions have very low collision frequency,

$$
\frac{v}{v \varepsilon / q r} \ll 1
$$

we can apply the well known procedure for the "banana" regime [11] i.e. we solve Eq.(7) by expanding the solution in $v$,

$$
f=f_{0}+f_{1} \ldots
$$

with the lowest expansion terms obeying the following equations,

$$
\begin{aligned}
& \frac{\partial \mathrm{f}_{0}}{\partial \theta}=0 \\
& \frac{\mathrm{u} \varepsilon}{\mathrm{qr}} \frac{\partial \mathrm{f}_{1}}{\partial \theta}=z v\left(\frac{v_{c}}{v}\right)^{3} \frac{\mathrm{u}}{\xi \mathrm{v}} \frac{\partial}{\partial \xi} \frac{\left(1-\xi^{2}\right) \mathrm{u}}{\xi_{\mathrm{v}}} \frac{\partial \mathrm{f}_{0}}{\partial \xi} \frac{\mathrm{B}}{\mathrm{B}_{0}}+\frac{v}{v^{2}} \frac{\partial}{\partial v}\left(v^{3}+v_{c}^{3}\right) \mathrm{f}_{0}+S_{0} \delta\left(v-v_{0}\right) .
\end{aligned}
$$

To find $f_{0}$, we apply the solubility condition, [11], which in essence means that $f_{1}$ is a periodic function of the poloidal angle,

$$
\left\langle\frac{\partial \mathrm{f}_{1}}{\partial \theta}\right\rangle \equiv \frac{1}{2 \pi} \oint \frac{\partial \mathrm{f}_{1}}{\partial \theta} \mathrm{d} \theta=0
$$

In our case all toroidally trapped particles are lost due to stochasticity. That is why the integration in Eq.(16) is performed along the passing orbit. The right-hand-side of Eq.(15), together with Eq.(16), gives the equation for the lowest order distribution,

$$
\frac{1}{s_{1}} \frac{\partial}{\partial \xi}\left(1-\xi^{2}\right) s_{2} \frac{\partial f_{0}}{\partial \xi}+\frac{1}{z} \frac{v}{v_{c}^{3}} \frac{\partial}{\partial v}\left(v^{3}+v_{c}^{3}\right) f_{0}+\frac{s_{0} \delta\left(v-v_{0}\right)}{v z}\left(\frac{v}{v_{c}}\right)^{3}=0,
$$

with 


$$
\begin{aligned}
& s_{1}=\left\langle\frac{v}{u}\right\rangle \xi, \\
& s_{2}=\left\langle\frac{u B}{v B_{0}}\right\rangle \frac{1}{\xi} \equiv\left\langle\frac{u}{v}\right\rangle \frac{1}{\xi} .
\end{aligned}
$$

The last approximation is made, because in this case we obtain the averaged quantitites in terms of elliptic integrals.

Eq.(17) is separable,

$$
f_{0}=\sum_{n} a_{n}(v) C_{n}(\xi)
$$

with $a_{n}$ and $C_{n}$ given by :

$$
\begin{aligned}
& \frac{1}{s_{1}} \frac{\partial}{\partial \xi}\left(1-\xi^{2}\right) s_{2} \frac{\partial C_{n}}{\partial \xi}=-\lambda_{n} C_{n}, \\
& -\lambda_{n} a_{n}+\frac{v}{z v_{c}^{3}} \frac{\partial}{\partial v}\left(v^{3}+v_{c}^{3}\right) a_{n}+\frac{\delta\left(v-v_{0}\right)}{v z}\left(\frac{v}{v_{c}}\right)^{3} K_{n}=0 .
\end{aligned}
$$

Here, the functions $C_{n}(\xi)$ are the orthogonal set of eigenfunctions, defined in the interval $\left(\xi_{t}, 1\right)$ with the boundary conditions imposed by Eq.(13),

$$
C_{n}\left(\xi_{1}\right)=0
$$

and $K_{n}$ being the weighted average of the source term,

$$
K_{n}=\frac{\int_{\xi_{1}}^{1} S_{0}(\xi) C_{n}(\xi) s_{1} d \xi}{\int_{\xi_{1}}^{1} C_{n}^{2}(\xi) s_{1} d \xi}
$$

Eq.(21) is trivially solved to give, 


$$
a_{n}=\frac{v_{0}^{2} K_{n} H\left(v_{0}-v\right)}{v\left(v^{3}+v_{c}^{3}\right)}\left(\frac{v_{0}}{v}\right)^{-\lambda_{0} z}\left(\frac{v^{3}+v_{c}^{3}}{v_{0}^{3}+v_{c}^{3}}\right)^{-\lambda_{0} z / 3},
$$

where $H(x)=1$ for $x>0$ and $H(x)=0$ for $x<0$.

The number of particles in the region $M$ given by Eq.(12) is changed due to processes described by the right-hand-side of Eq.(7). These are: the source at $v=v_{0}$, escaping through the boundary $\xi=\xi_{t}$ due to pitch-angle scattering, and crossing of the boundary $v=v_{s}$ due to slowing down. The rate of the density change averaged over the magnetic surface is then given by

$$
\left\langle\frac{\partial N}{\partial t}\right\rangle=\left\langle\int_{M}\left(S_{0} \delta\left(v-v_{0}\right)+C_{p}(f)+C_{v}(f)\right) d^{3} v\right\rangle .
$$

The particles can go through the boundary $\xi=\xi_{\mathrm{t}}$ of the region $\mathrm{M}$ only due to pitch angle scattering. Then they become subject to immediate stochastic diffusion. That is why the stochastic loss flux is

$$
\Gamma_{n}=-\left\langle\int_{M} C_{p}(f) d^{3} v\right\rangle
$$

This is the flux of particles born outside the stochastic region, but lost stochastically due to the pitch scattering into the stochastic region. For simplicity, we call it just the stochastic flux. For an integrand which is symmetric with respect to the sign of the parallel velocity, the element of volume $d^{3} v$ and integration limits in Eqs.(25) and (26) are

$$
\int_{M} d^{3} v=4 \pi \int_{v_{0}}^{+\infty} v^{3} d v \int_{\xi_{1}}^{1} \frac{\xi d \xi}{|u|}
$$


Eqs.(7), (25) and (26) permit us to represent $\Gamma_{\mathrm{st}}$ in the form,

$$
\Gamma_{s t}=-4 \pi v z v_{c}^{3} \int_{v_{1}}^{+\infty} \frac{d v}{v} \int_{\xi_{1}}^{1} d \xi \frac{\partial}{\partial \xi}\left(1-\xi^{2}\right) s_{2} \frac{\partial f}{\partial \xi},
$$

which, according to Eq.(20), is

$$
\Gamma_{\mathrm{st}}=4 \pi v z v_{c}^{3} \sum_{n} \lambda_{n} \int_{v_{1}}^{+\infty} \frac{d v}{v} a_{n} \int_{\xi_{1}}^{1} C_{n}(\xi) s_{1} d \xi
$$

through the lowest order. Substituting Eq.(24) into Eq.(29), we finally obtain

$$
\Gamma_{\mathrm{at}}=4 \pi v z v_{\mathrm{c}}^{3} \sum_{\mathrm{n}} \lambda_{\mathrm{n}} \mathrm{Z}_{\mathrm{n}} \mathrm{V}_{\mathrm{n}}
$$

where

$$
Z_{n}=x_{c}^{3} \int_{x_{d}}^{1} \frac{d x}{x^{3}+x_{c}^{3}} x^{\lambda_{c} 2-1}\left(\frac{x^{3}+x_{c}^{3}}{1+x}\right)^{-\lambda_{c} z / 3}
$$

with $x_{a}=v_{s} / v_{0}, x_{c}=v_{c} / v_{0}$ and

$$
V_{n}=\frac{\int_{\xi_{1}}^{1} S_{0} s_{1} C_{n} d \xi \int_{\xi_{1}}^{1} s_{1} C_{n} d \xi}{\int_{\xi_{1}}^{1} s_{1} C_{n}^{2} d \xi}
$$

The stochastic loss flux should be compared with the flux entering the region $\left\{0 \leq v \leq v_{z}, 0 \leq \xi \leq 1\right\}$ due to the production of ions by the source term,

$$
\Gamma_{0}=4 \pi \int_{0}^{+\infty} v \delta\left(v-v_{0}\right) d v \int_{0}^{v} S_{0} d u=4 \pi v_{0} \int_{0}^{v_{0}} S_{0} d u
$$


Then the relative stochastic loss is

$$
\gamma_{\mathrm{sz}}=\Gamma_{\mathrm{a}} / \Gamma_{0} .
$$

For the case of the isotropic source, when $S_{0}$ does not depend on $\xi$, which is the case for alpha particles, we have $\Gamma_{0}=4 \pi S_{0} v_{0}^{2}$. Then $\gamma_{s t}$ takes the form:

$$
\gamma_{\mathrm{z}}=\mathrm{z} \sum_{\mathrm{a}} \lambda_{\mathrm{n}} \mathrm{Z}_{\mathrm{n}} \mathrm{Q}_{\mathrm{n}}
$$

where

$$
Q_{n}=\frac{\left(\int_{\xi}^{1} s_{1} C_{n} d \xi\right)^{2}}{\int_{\xi_{1}}^{1} s_{1} C_{n}^{2} d \xi} .
$$

The energy loss is evaluated in an analogous way. For an isotropic source the relative energy loss due to the stochastic mechanism is then:

$$
\gamma_{a}^{E}=2 \sum_{a} \lambda_{a} Y_{a} Q_{n}
$$

where $Y_{n}$ is an integral similar to $Z_{n}$, given by Eq.(31),

$$
Y_{n}=x_{c}^{3} \int_{x_{1}}^{1} \frac{d x}{x^{3}+x_{c}^{3}} x^{\lambda_{e} z+1}\left(\frac{x^{3}+x_{c}^{3}}{1+x}\right)^{-\lambda_{2} z^{2 / 3}}
$$




\section{Results}

In order to retain the effects of the finite $\varepsilon$, we found the functions $C_{n}(\xi)$ - given by Eq.(20) - numerically. The numerical method we used, is quite standard. We used the shooting method [15], starting from the point $x \equiv 1-\xi^{2}=x_{\min } \approx 0$. To find the initial values of the solution and its derivative, we expanded the coefficients of Eq.(20) near $x=0$ and solved it analytically. The initial guess for $\lambda$ was done by the WKB method, i.e. by setting the coefficients of Eq.(20) equal to their average. To propagate the solution of the differential equation, we used the 2-d order Runge-Kutta method [15]. New guess for $\lambda$ was performed by matching the solution to the boundary condition at $x_{\max }=1-\xi_{1}^{2}$ (Newton-Raphson method [15]).

Everywhere, we left only the first order corrections in the toroidicity, $\delta$. The finite, although small, value of $\delta$ affects the results and we found it interesting to follow its influence. The case $\delta=0$ can be solved analytically (like in [12-14]). Since this analytical results is represented as an expansion in Legendre polynomials and not very handy, we included it in the general case, investigated numerically.

Fig.2 represents the solutions of Eq.(20) for toroidicity $\varepsilon=0.15$. This value approximately corresponds to ITER [16] toroidicity at $r=a / 2$.

With the solutions $C_{n}(x)$ found, we evaluated the integrals occurring in the stochastic flux. We present results corresponding to the alpha particles born in D-T reactions with the energy $3.5 \mathrm{Mev}$. Fig. 3 show the dependencies of the relative stochastic

fluxes of the particles and energy, $\gamma_{\mathrm{st}}$ and $\underset{\gamma_{\mathrm{t}}}{\mathrm{E}}$, on the ripple magnitude $\delta$. Note again, that those are the additional fluxes due to pitch-angle scattering into stochastic region. The dependancies were found numerically for the following data: $z=1, B=10 T$, $\mathrm{Te}=20 \mathrm{kev}, \mathrm{q}=3, \partial \mathrm{q} / \partial \mathrm{r}=0.06 \mathrm{~cm}^{-1}, \mathrm{~N}=16$. These roughly correspond to ITER [16] parameters. 
It is interesting, that already at relatively small values of ripple, the dependencies $\gamma_{s t}(\delta)$ and $\gamma_{t l}^{E}(\delta)$ turn into a kind of plateau. This happens because the lower limit in the integrals given by Eqs.(31) and (38) becomes very small, so that its change does not effect their values. The asymptotic value (at large $\delta$ or at $v_{s}=0$ ) of the relative particle loss corresponds to the fraction of passing particles, $\Delta_{p} \cong\left(1-\xi_{v}\right)$, with all of them having been scattered into the loss cone. Since the particles, which are born toroidally trapped, are also lost, the asymptotic regime corresponds to a situtation when all particles are being lost. The asymptotic value of the energy loss can not be found by similar consideration because the energy is not conserved in the slowing down. This asymptotic value of the lost energy depends only on the value of toroidicity and the electron temperature and can be easily evaluated with use of Eqs.(37) and (38) where $x_{s}$ should be set zero. Actually, this "asymptotic" regime is realized for most of the devices since the value of ripple usually is of order $10^{-2}$. Fig. 4 shows the dependencies of the asymptotic values of fractions of the energy and particles born outside the stochastic region and then lost stochastically, $\gamma_{s t}(\varepsilon)$ and $\gamma_{s t}^{E}(\varepsilon)$ as functions of the toroidicity, $\varepsilon$, at $T_{e}=20 \mathrm{kev}$. These particles get into the stochastic region due to the pitch-angle scattering. Since in this regime the region $M$ becomes the whole space at $\varepsilon=0, M=\left\{0 \leq v \leq v_{0}, 0 \leq \xi \leq 1\right\}$, $\gamma_{a}(\varepsilon)$ tends to 1 as $\varepsilon$ goes to zero.

Note, that to obtain the total relative energy loss, $\gamma_{s t}^{\text {tot }}(\varepsilon)$, we should add the fraction of the energy immediately lost in the toroidally trapped region, $\Delta_{t}(\varepsilon) \cong(\varepsilon)^{1 / 2}$, to $\underset{\gamma_{s t}}{E}(\varepsilon)$. This amounts, for example, to $\gamma_{s t}^{\text {tot }}(\varepsilon) \cong 0.6$ at $\varepsilon=0.15, T=20 \mathrm{kev}$. As we have already mentioned, the same concerns the particle loss: we should add the fraction of the toroidally trapped particles (lost at their birth) $\Delta_{t}$ to $\gamma_{s t}(\delta)$ and $\gamma_{n t}(\varepsilon)$ in order to obtain the total loss. Since we study the stationary solutions, in the limit $v_{S}=0$ we shall obtain $\gamma_{\text {lot }}(\varepsilon)=\gamma_{\mathrm{st}}(\varepsilon)+\Delta_{1}=1$ 
Thus, we have presented the solution of the drift kinetic equation for the fast ions in the tokamak magnetic configuration with both collisional (slowing down and pitch angle scattering) and collisionless (stochastic diffusion which creates the loss cone) effects taken into account. The stochastic losses have been evaluated by solving the eigenfunction problem numerically. The loss due to stochastic diffusion is dependent on values of the parameters involved in the problem (threshold energy for the onset if the chaotic behavior). But in the "typical" situation, i.e. close to the ITER configuration, the energy contribution of the alpha particle to the background plasma constitutes about $40 \%$ although all the particles can be lost due to the rapid stochastic diffusion.

These results agree qualitatively with the results obtained by Monte-Carlo codes [6,7], though give higher particle loss then that given by RIPLOS [2] (particle loss constitutes about $10 \%$, therefore the energy loss in those results should be even smaller). It is not surprising that RIPLOS gives lower values of the losses, because the collisions are not included there. Our study thus shows that pitch-angle scattering changes the value of the stochastic losses by amount $\gamma_{s t}$ which can be quite large (Fig.3). Our results are comparable, but larger (factor of $1.5-2$ ) then those given by Tani $[5]$ and in $[4,7]$ (energy loss up to 30\%), where the full Monte Carlo simulation (with the Landau collision operator and guiding center equations) was performed. It is also not surprising, because in reality the stochastic losses are not so rapid, and the particles born inside and scattered into stochastic region are confined for some finite time. Note, that to compare the results qualitatively, we use the saturation values of the losses, because it is not clear how to compare the losses at particular values of ripple (we calculate the losses at particular minor radius, while in other papers the bulk loss is calculated as a function of ripple at the edge). 
Figure captions

Fig.1 The velocity space of the fast ions. The high energy $\left(v_{s}<v<v_{0}\right)$ toroidally trapped particles $\left(0<\xi<\xi_{0}\right)$ rapidly leave the system. The solution of the kinetic equation is considered in the region $M=\left(v_{S}<v<v_{0}, \xi_{l}<\xi<1\right)$. The number of the particles in $M$ is changed due to the source at $\mathrm{v}=\mathrm{v}_{0}$, slowing down and pitch angle scattering.

Fig.2 The eigenfunctions $C_{n}(\xi)(n=1-5)$ at $\varepsilon=0.15$. The eigenvalues are: $\lambda_{n}=13.609$, $20.3834,50.4548,93.8008,220.3749 \ldots$...

Fig.3a The relative stochastic losses of alpha particles, born outside of stochastic region and scattered into it, versus magnitude of the ripple, $\delta$. The main parameters of the case under consideration are: $z=1, B=10 T, T e=20 \mathrm{kev}, q=3, \partial q / \partial \mathrm{r}=0.06 \mathrm{~cm}^{-1}, N=16, \varepsilon=0.15$.

Fig.3b The relative stochastic losses of energy of alphas, born outside of stochastic region and scattered into it, versus magnitude of the ripple, $\delta$. The main parameters of the case under consideration are: $z=1, B=10 T, T e=20 \mathrm{kev}, q=3, \partial q / \partial r=0.06 \mathrm{~cm}^{-1}, N=16$, $\varepsilon=0.15$.

Fig.4a The relative stochastic loss of alpha particles, born outside of stochastic region and scattered into it in the process of slowing down, versus toroidicity $\varepsilon$, for the case when all of them (including toroidally trapped particles lost at birth) are stochastically lost, $\mathrm{T}_{\mathrm{e}}=20 \mathrm{kev}$.

Fig.4b The relative stochastic loss of energy of the particles, born outside of stochastic region and scattered into it in the process of slowing down, versus toroidicity $\varepsilon$, for the case when all of them (including toroidally trapped particles lost at birth) are stochastically lost, $\mathrm{T}_{\mathrm{e}}=20 \mathrm{kev}$. 
References:

1. H.P. Furth, R.J. Goldston, S.J. Zweben, D.J. Sigmar, Nuclear Fusion 30, 1799 (1990).

2. R.B. White, H.E. Mynick, Phys. Fluids B 1, 980 (1989).

3. R.J. Goldston, R.B. White, A.H. Boozer, Phys. Rev. Let. 47, 647 (1981).

4. P.N. Yushmanov, in Reviews of Plasma Physics, edited by B.B.Kadomtsev (Consultants Bureau, New York, 1991), Vol.16, p.117.

5. K. Tani, T. Takizuka, M. Azumi, H. Kishimoto, Nuclear Fusion 23, 657 (1983).

6. L.M. Hively, Nuclear Fusion 24, 779 (1984).

7. F.S.Zajtsev, A.P.Smimov, P.N.Yushmanov, Nuclear Fusion 26, 1311 (1986).

8. R.B. White, A.H. Boozer, R.J. Goldston, R. Hay, J. Albert, C.F.F. Karney, in Plasma Physics and Controlled Nuclear Fusion Research, 1982, Proc. of the 9th International Conference (IAEA, Vienna, 1983), Vol.II, p.391.

9. K. Tani, T. Takizuka, M. Azumi, in Proceedings of the Workshop on 'Statistical Plasma Physics,' US-Japan Joint Institute for Fusion Theory Program (Institute of Plasma Physics, Nagoya 16, 199 (1976).

10. S.V. Bulanov, L.M. Kovrizhnykh, S.G. Shasharina, Fusion Technology 18, 403 (1990).

11. P.H. Rutherford, Phys. Fluids 13, 482 (1970)

12. J.G. Cordey, Nuclear Fusion 16, 499 (1976).

13. C.T. Hsu, P.J. Catto, D.J. Sigmar, Phys. Fluids B 2, 280 (1990).

14. M. Taguchi, Plasma Physics and Controlled Fusiom 33, 859 (1991).

15. W.H. Press e.a., Numerical Recipes, Cambridge University Press, Cambridge (1986).

16. INTERNATIONAL ATOMIC ENERGY AGENCY, ITER Conceptual Design: Interim Report, ITER Documentational Series, No. 7, LAEA, Vienna (1990). 


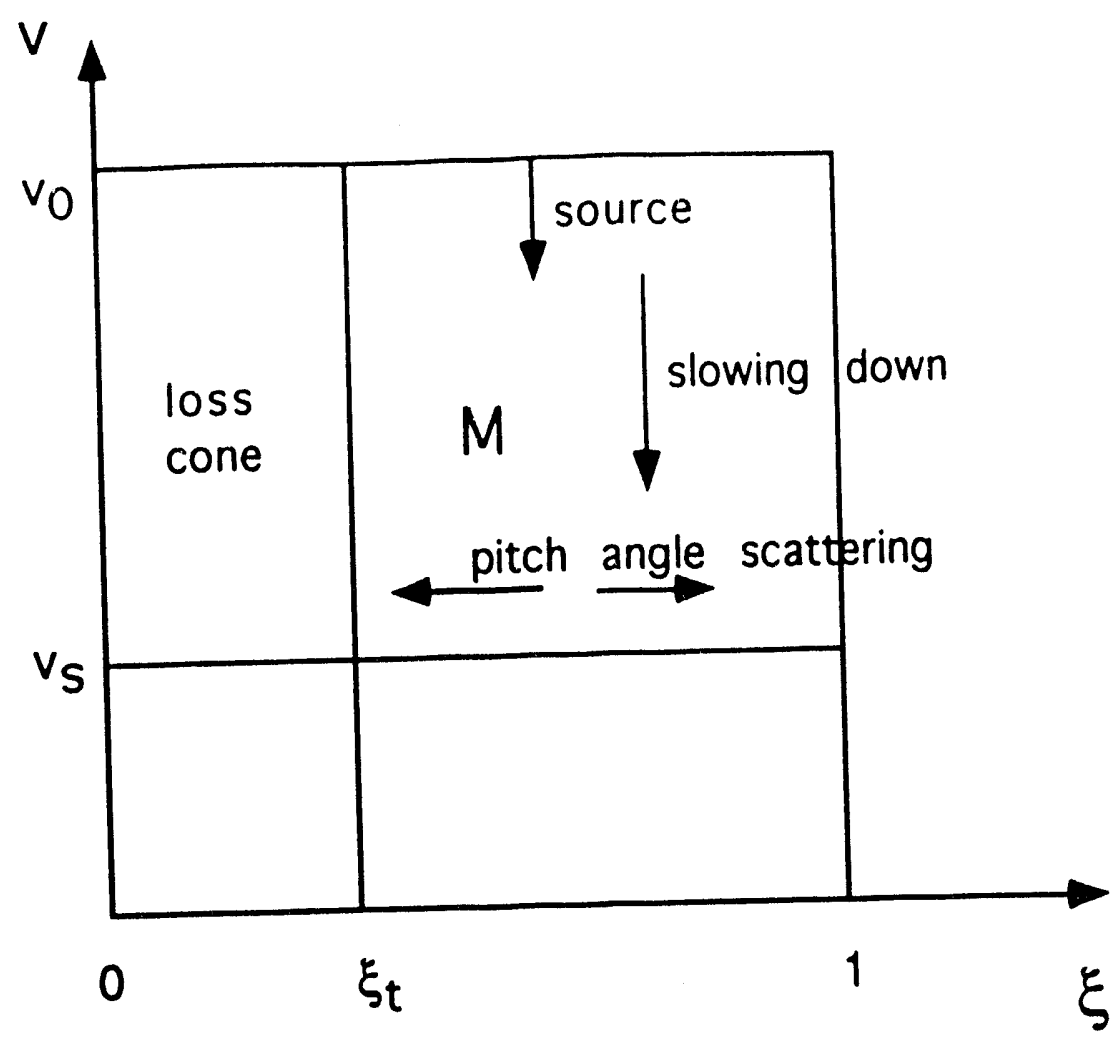

Fig. 1 


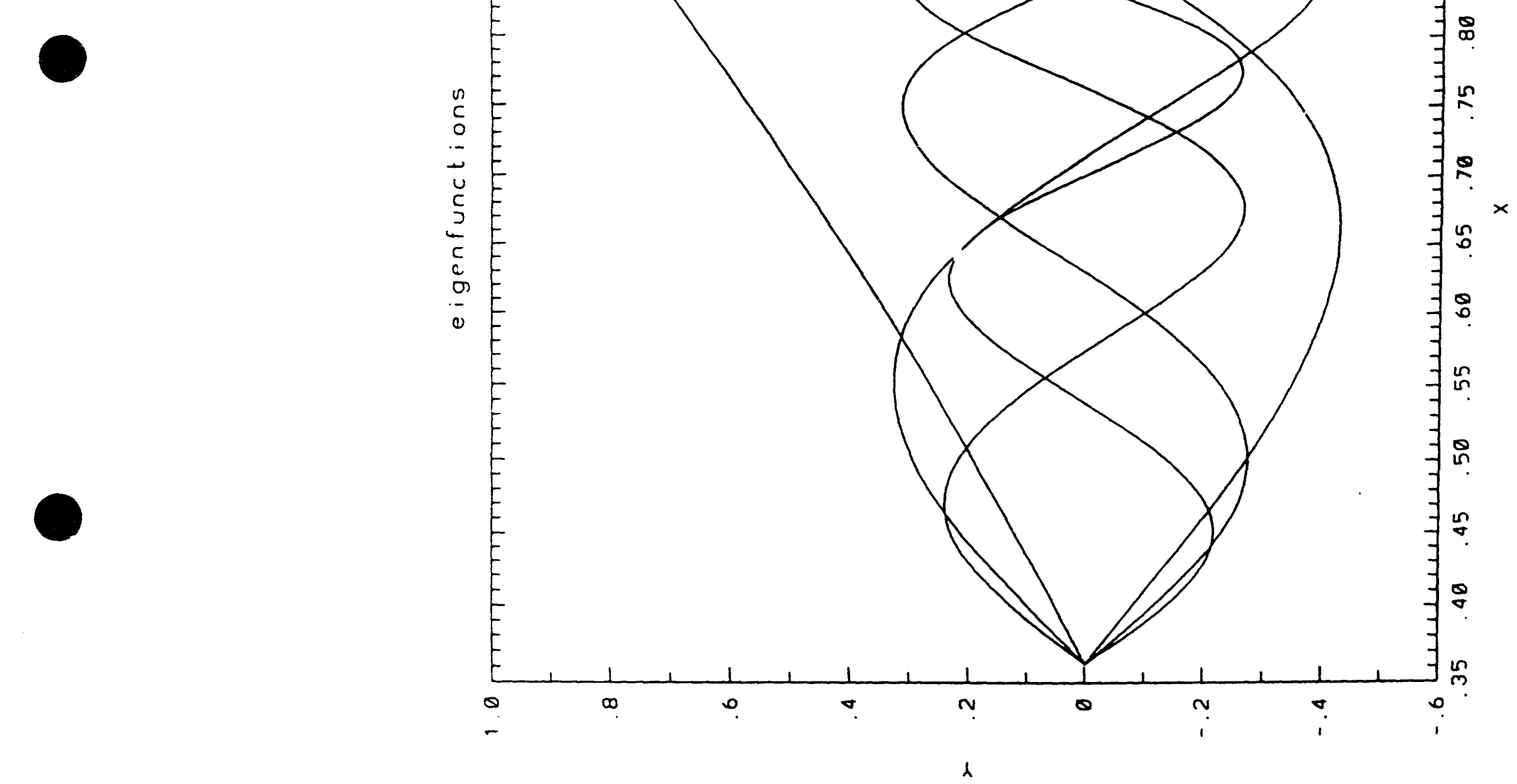

ํㅜㅁ 

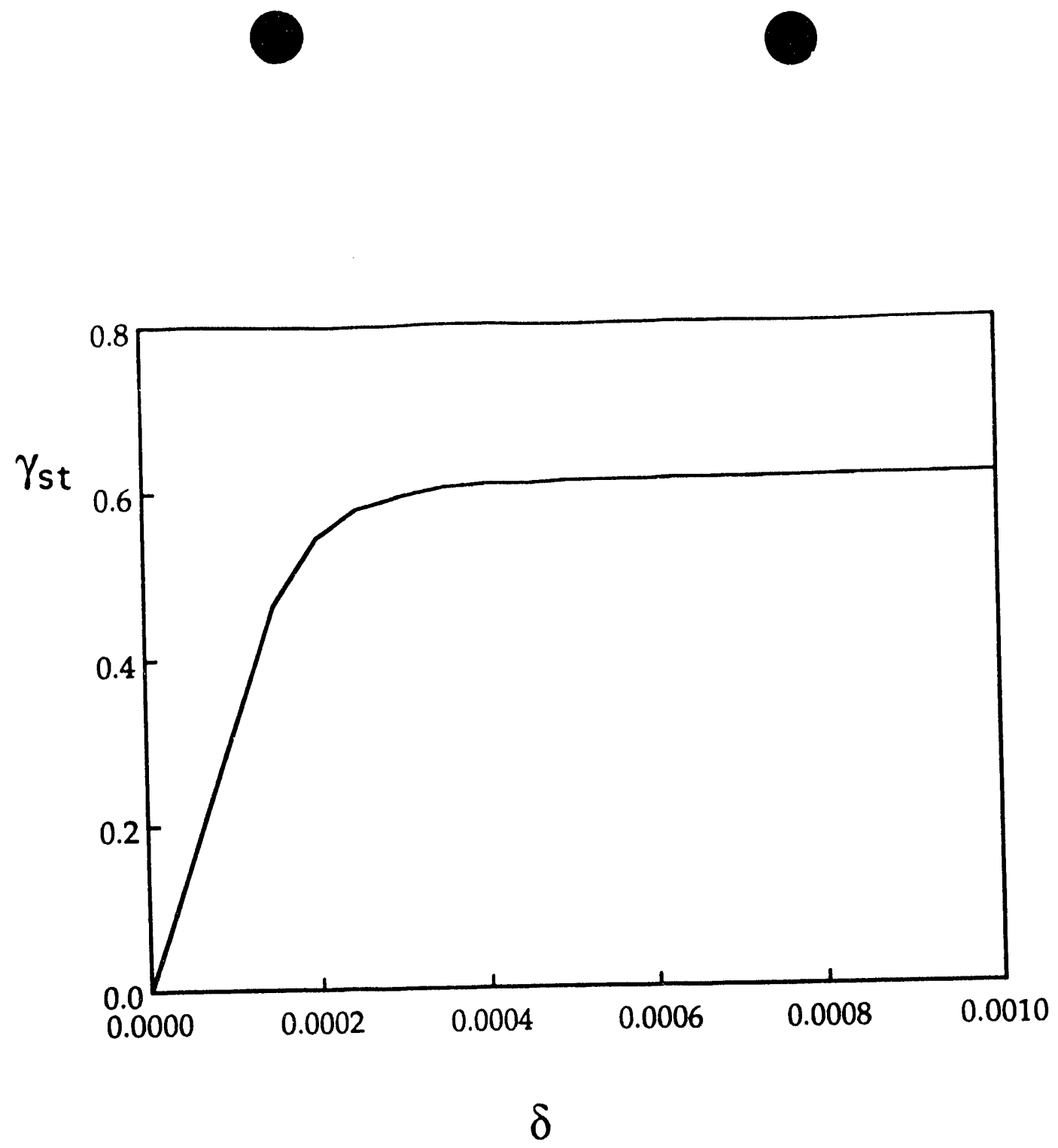

Fig.3a 


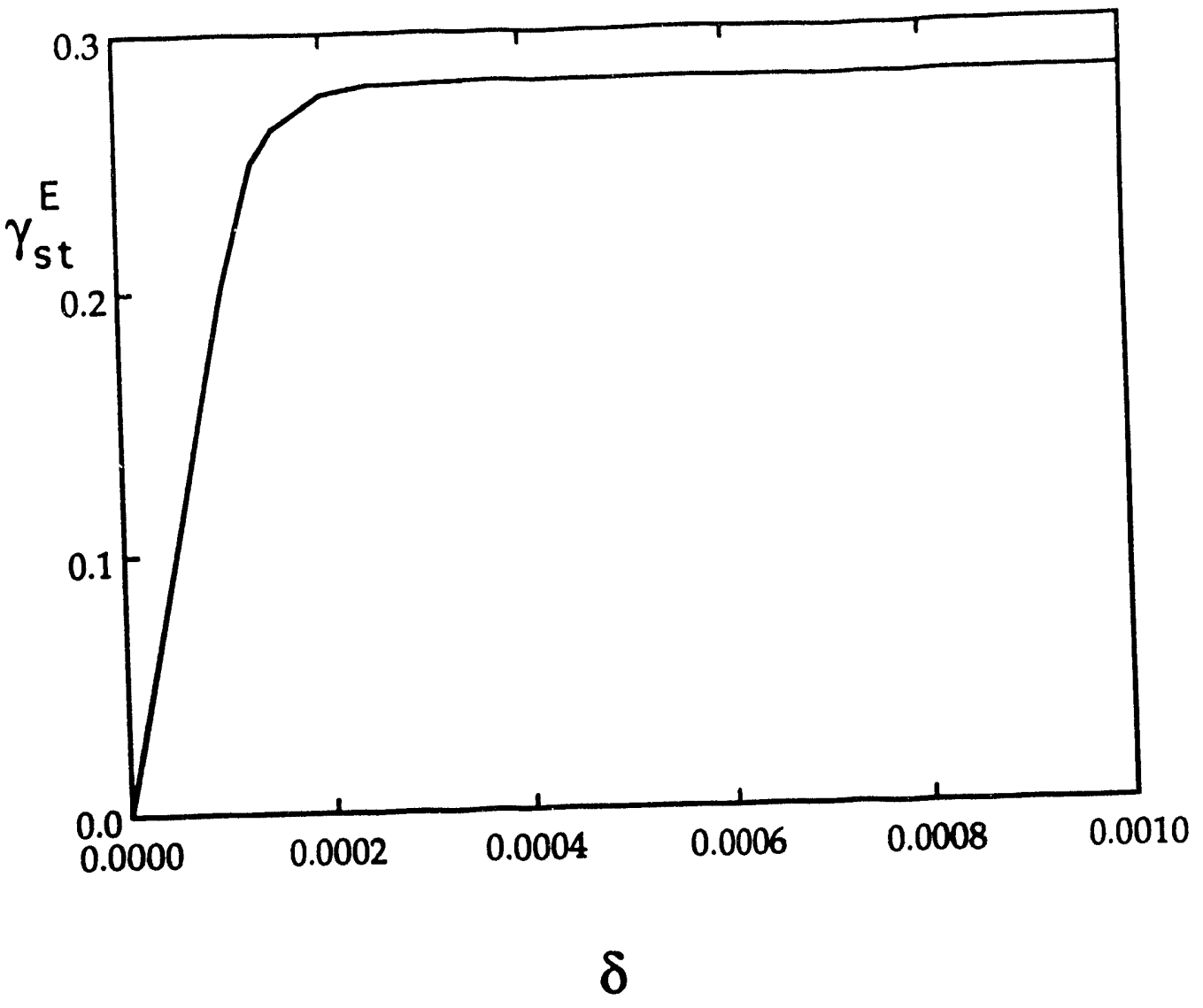

Fig.3b 
ํㅜㅁ

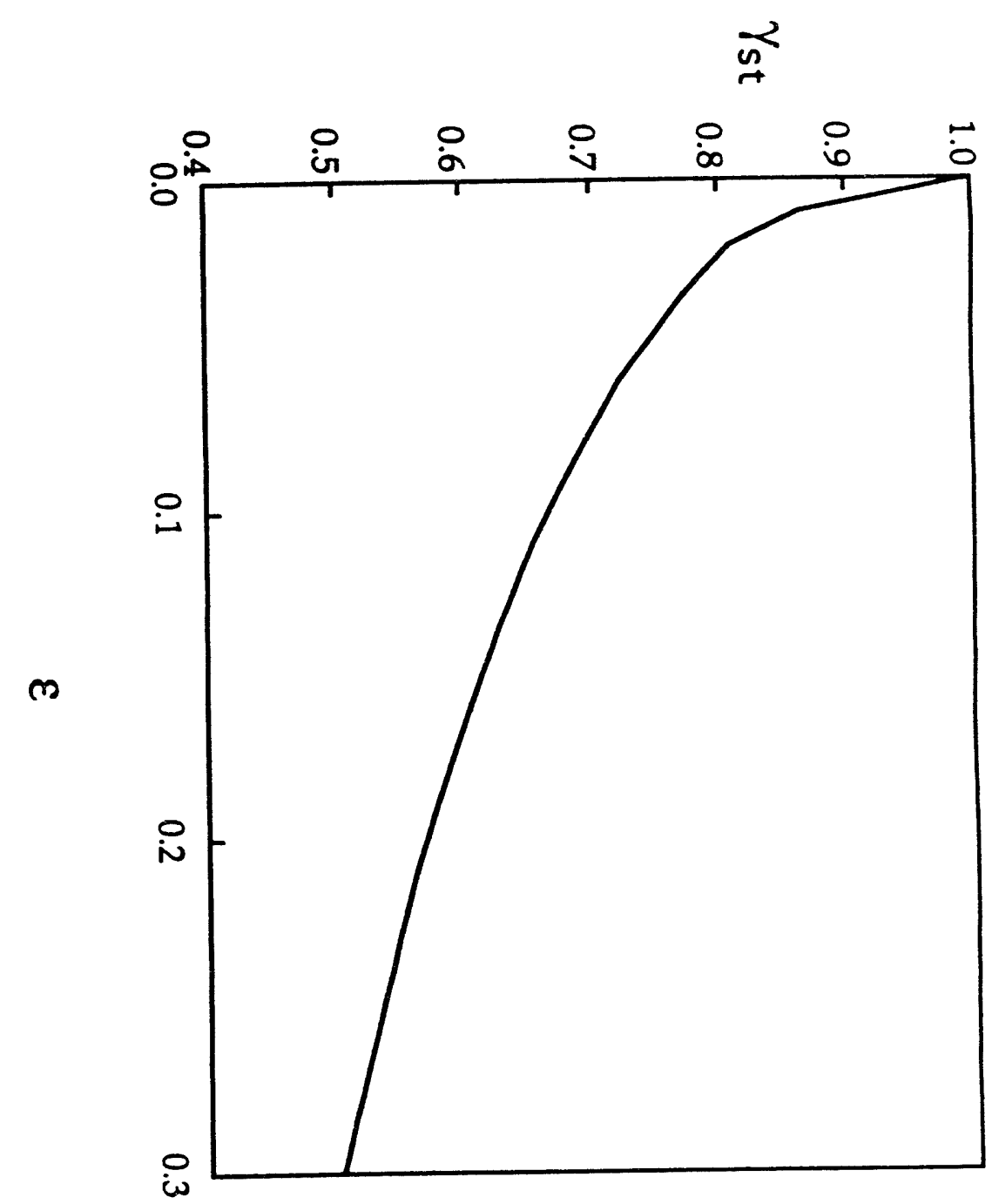




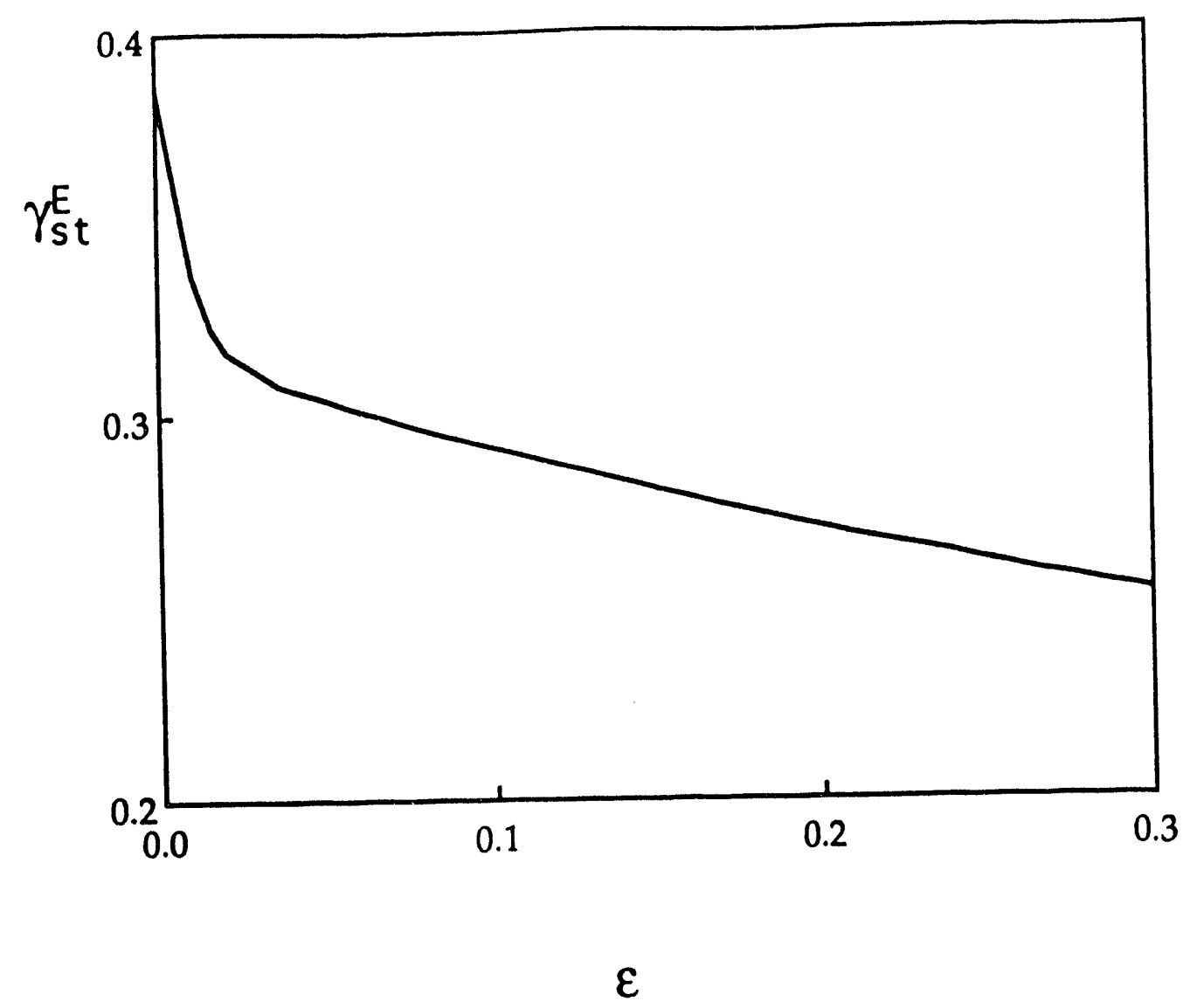

Fig. $4 b$ 
Appendix C. Transport in Non-axisymmetric Tori with Up-Down Asymmetry 
Transport in Non-axisymmetric Tori with Up-Down Asymmetry

$$
\text { P.N.Yushmanov, " John R.Cary, "* S.G.Shasharina*" }
$$

\begin{abstract}
Transport phenomena arising due to up-down asymmetry of ripple and helical perturbations of the magnetic field in tokamaks and stellarators are investigated. Accounting for this asymmetry is important for the devices with vertically displaced magnetic axes or with a single-null divertor. The asymmetry manifests itself in different magnitudes of the ripple or helical perturbation in the up and down parts of the magnetic surface. Due to this difference, the toroidally trapped particles acquire a net radial drift. We study the collisionless dynamics of the particles in such configurations as well as solutions of the kinetic equation. We show that in spite of the net radial drift, the up-down asymmetry does not lead to enhanced diffusive transport, compared to the conventional ripple transport, although the convective loss at low collisionality might be large.
\end{abstract}

- Instiwute for Fusion Studies and Fusion Research Center, The University of Texas at Austin, Austin, Texas 78712

** University of Colorado, Boulder, CO 80309-0391 


\section{Introduction}

The most important classical channel of the particle loss in toroidal magnetic systems is the ripple diffusion of the toroidally trapped particles [1]. It arises due to distortion of the axial symmetry of the torus by the finite perturbation of the toroidal magnetic field. Hence, the ripple diffusion has been a subject of intense studies for the ideal configurations [1-7] as well as for concrete devices [8-11].

The best solution of the problem is to diminish the toroidal asymmetry. But decreasing the ripple magnitude is quite expensive, because it requires the volume of the coils to be increased. That is why studies were aimed mostly to estimate the critical magnitude of ripple in order to find the balance between allowed losses and a reasonable design of the device. It is not only the loss value which should be known. The distribution of the particle and energy flux on the chamber walls is also very important. Here, the influence of the geometry and the features of the orbits come into play. Although the main loss of bulk plasma is expected to be governed by anomalous mechanisms, the classical loss of high energy particles (alpha-particles, NBI-ions, tail particles) is comparable with the anomalous one.

In this regards, any new breaking of the symmetry of the magnetic field should be thoroughly investigated, as it might lead to an additional loss channel and affect the confining properties of the device.

In the present paper we investigate the neoclassical transport associated with updown asymmetry of the magnetic field in tokamaks and stellarators. In stellarators, the ripple magnitude is usually considered to be some function of the minor radius. In several cases, helical modulation dependent on the poloidal angle was studied (see $[5,12]$ ), but the up-down symmetry was always implied both for stellarators and tokamaks. But it is not always the case in the real experiments. Substantial up-down asymmetry can arise due to 
even rather small vertical displacement of plasma with respect to the center of the chamber $[13,14]$. Besides, all magnetic configurations with a single null divertor are naturally asymmetric from the very beginning. In such cases, the depth of the local magnetic wells, $\Delta$, is different in the upper and lower parts of the magnetic surfaces. The value of this asymmetry, estimated in Secs .2 and 3 , can be of order unity, $\left(\Delta^{+}-\Delta^{-}\right) /\left(\Delta^{+}+\Delta^{-}\right)=1$. In these magnetic configurations, the toroidally trapped particles - those with reflection points located in the region where the local magnetic wells exist - acquire a net radial velocity. This velocity, evaluated in Sec. 4 , is related to the toroidal drift velocity $v_{d}$ and the toroidicity $\varepsilon_{\mathrm{t}}, \dot{r}_{\mathrm{t}}=v_{\mathrm{d}} \sqrt{\frac{\Delta}{\varepsilon_{\mathrm{t}}}}$ and could lead to an additional transport. This is the case at low collisionality, when the presence of the physical boundaries is essential and the losses due to new net drift motion are rapid. The situation is different at higher collisionality, when the radial displacement of the particles between the collisions is small. In this case, the rigorous analysis of the bounce-averaged kinetic equation (Sec. 5) shows that the additional diffusive flux of the toroidally trapped particles is totally compensated by the change in the flux of the locally trapped particles, so that the transport remains unchanged. Conclusions on the subject are presented in Sec. 6.

\section{Up-down asymmetry of the ripple}

Neoclassical transport in the toroidal magnetic confinement devices is determined mostly by the magnetic field strength,

$$
B=B_{0}\left[1-\varepsilon_{\mathrm{t}} \cos (\theta)-\varepsilon_{\mathrm{r}} \cos (N \phi-l \theta)\right]
$$

Here, $\varepsilon_{t}=r / R_{0}$ is the inverse aspect ratio (toroidicity), $\varepsilon_{r}$ is the ripple magnitude (or the helical modulation, for the stellarator); $l$ and $N$ (usually $N \gg l$ ) are the poloidal and toroidal mode numbers for a stellarator, while $l=0$ for number for a tokamak; $\theta$ and $\phi$ are the 
poloidal and toroidal angles, which are related to each other on the magnetic field lines via the rotational transform, $d \phi / d \theta=-1 / t=-q ; r$ and $R=R_{0}\left(1+\varepsilon_{l} \cos (\theta)\right)$ are the minor and major radius.

Let us estimate the influence of the vertical displacement of the plasma to the magnitude of ripple. When the magnetic field in the tokamak is created by uniformly distributed circular coils, the contours of constant ripple strength $\varepsilon_{\mathrm{r}}$, are approximately circles with the centers shifted towards the major axis of the torus. In this case, we have the following equation for the dependence of the ripple amplitude on coordinates [4].

$$
\varepsilon_{r}=\varepsilon_{\min } I_{0}\left(\frac{N b}{R_{0}-L_{0}}\right) \text {, }
$$

where $I_{0}$ is the modified Bessel function, $\varepsilon_{\min }$ and $L_{0}$ are parameters determined by the shape of the coils, $b$ is the radius of the circle $\varepsilon_{r}=$ constant,

$$
b=\left[\left(r \cos (\theta)+L_{0}\right)^{2}+(r \sin (\theta)+\Delta z)^{2}\right]^{1 / 2}\left(\frac{R_{0}-L_{0}}{R_{0}+r \cos (\theta)}\right)^{1 / 2},
$$

with $\Delta z$ being the vertical displacement of plasma. The approximation $L_{0}(x) \cong \exp (x)$, $\mathrm{L}_{0} \ll \mathrm{a}, \mathrm{R}_{0}, \Delta \mathrm{z}<\mathrm{a}$, where $\mathrm{a}$ is the minor radius of the torus, gives then

$$
\begin{aligned}
& \varepsilon_{r}=\varepsilon_{r}^{r j m} \exp \left(\frac{N \Delta z \sin (\theta)}{R_{0}}\right), \\
& \frac{\varepsilon_{r}(r, \pi / 2)}{\varepsilon_{r}(r,-\pi / 2)}=\exp \left(\frac{2 N \Delta z}{R_{0}}\right) .
\end{aligned}
$$

For reasonable parameters, $N=16$ and $\Delta z / R_{0}=0.02$, the ripple varies by a factor of two over the plasma volume. Typical contours of the ripple amplitude for the shifted plasma are depicted on Fig.1. 
The effective depth of the local masnetic well, $\Delta$, depends not only on the magnitude of ripple. It also depends on parameter $\alpha$ (see $[4,5])$ via

$$
\Delta=\varepsilon_{r}\left[\sqrt{1-\alpha^{2}}-\alpha\left(\frac{\pi}{2}-\sin ^{-1}(\alpha)\right)\right]
$$

where

$$
\alpha \equiv \frac{\left(B \cdot \mathbf{e}_{R}\right)}{B N \varepsilon_{\mathrm{r}}}=\frac{\varepsilon_{\mathrm{q}} \sin (\theta)}{\varepsilon_{\mathrm{r}} \mathrm{Nq}},
$$

where $e_{R}$ is the unit vector directed along the major radius. One can see that, in the configurations with a single null divertor, due to decreasing of $\left(B e_{R}\right) / B$ in the vicinity of the X-point, the vertical asymmetry in the value of $\Delta$ can be of order of unity.

The magnetic field (1) has the same form for tokamaks and stellarators [5], although the ripple magnitudes are different functions of coordinates. Since we do not use any particular form for these functions, all the results obtained below are applicable to both systems, i.e. to the toroidally asymmetric tori with up-down asymmetry of the toroidal perturbation of the magnetic field.

\section{Trajectories of the particles in the up-down asymmetric tori.}

Let us consider the effect which the asymmetric ripple produces. There are essentially three types of motion in the rippled magnetic field: passing, toroidally trapped and locally trapped particles. As the magnetic wells are not generally constant, transitions between locally and toroidally trapped states occur, so that there exist transition particles which "live" part of the time in toroidally and locally trapped states $[4,15,16]$. In a vertically symmetric magnetic field, bananas (we use the term "banana" as short for "toroidally trapped") are closed on average, so they have no systematic radial displacement. 
Slight unclosing of the banana trajectories is related to the different toroidal phases of the reflection points of the toroidally trappe- particles. Transport associated with this phase uncompensation in radial displacement is studied in $[3,4,5]$. Averaging over this phase gives zero radial displacement.

The situation is quite different when the magnetic wells confining the locally trapped particles are vertically asymmetric. Due to the asymmetry, the toroidally trapped particle reflects in the upper and lower parts of the torus at points which are not symmetric and lie on the different magnetic surface (see Fig.2a,b). This difference persists after phase averaging. As a result, the banana acquires a net inward or outward drift. The sign of the net flux depends on the directions of the drift velocity and the gradient of the ripple amplitude. Let us assume, for example, that the ripple is bigger in the upper plane, and the drift velocity is directed downwards. Then the upper reflection point is closer to the midplane then the lower one, and the banana, as a whole, moves outwards. If we change the sign of the drift or make the ripple larger in the lower plane, the banana drifts inwards.

To balance the net banana flux and maintain Liouville's theorem, a counter-flux should exist. It cannot be provided by the particles in the same state, because all of them have the same average velocity. The counter-flux can be provided only by the particles in the locally trapped state which then collisionlessly detrap and become the toroidally trapped. This means that the new phenomena concem only the transition particles which drift inwards in one state and outwards in another. The resulting drift of the whole transition orbit is shown on Fig.2a,b. The non-transitional particles cannot experience non-zero average drift, because the invariant group of the particles cannot have a sink without having a source, and vice-a versa. Only the transition orbits provide this kind of circulation. This means that, to obtain the new transport, the local wells should exist in the reflection points of the banana particles, i.e. the condition, $\alpha \leq 1$, must be fulfilled in some region of the cross-section, where $\alpha$ is given by Eq.(7). 
According to this picture, the toroidally trapped region of the velocity space has sources and sinks on the boundary with locally trapped region, meanwhile the locally trapped region has the corresponding sinks and sources. Note that in vertically symmetric case, the sinks and sources of the transition orbits in the upper and lower parts compensate each other.

The balance between the incoming and outcoming fluxes actually determines the relationship between the drift velocities in toroidally and locally trapped states,

$$
\dot{r}_{t} f_{t} \approx v_{d} f_{s}
$$

where $\dot{r}_{1}$ and $v_{d}$ is the average drift velocity in the toroidally and locally trapped state,

$$
\mathrm{v}_{\mathrm{d}}=\frac{\mu \mathrm{B}_{0}}{\mathrm{R}_{0} \Omega}
$$

with $\Omega$ the gyro frequency, $\Omega=\frac{e B_{0}}{m c}$, and

$$
\mathrm{f}_{\mathrm{t}} \approx \sqrt{\varepsilon_{\mathrm{t}}}
$$

and

$$
\mathrm{f}_{\mathrm{r}} \approx \sqrt{\varepsilon_{\mathrm{r}}}
$$

are the relative phase space volumes corresponding to the toroidally and locally trapped states.

These transition particles can be lost either diffusively or convectively, as is determined by the characteristic time and length scales. If the drift radial displacement between the collisions is much smaller then the minor radius, the loss is diffusive. This displacement is $\frac{\dot{r}_{t}}{v_{t}^{e f}}=\sqrt{\frac{\varepsilon_{s}}{\varepsilon_{t}}} v_{d} / v_{t}^{\text {eff }}$ in the toroidally trapped state, while the displacement of the locally trapped particles is $v_{d} / v_{t}^{\text {fff }}$. Since the transition particles have two branches 
with different radial drift and different collisionality, the losses are diffusive, if the following two conditions are fulfilled simultaneously,

$$
\begin{aligned}
& v_{t}^{\text {eff }} \gg \sqrt{\frac{\varepsilon_{r}}{\varepsilon_{1}}} \frac{v_{d}}{a} \\
& v_{t}^{\text {eff }} \gg \frac{v_{d}}{a},
\end{aligned}
$$

with $v_{k}^{\text {eff }}$ the effective collision frequency for scattering out of the state,

$$
v_{k}^{\text {eff }}=\frac{v}{f_{k}^{2}}
$$

and $f_{k}$ given in Eqs.(10) and (11). Eqs.(12) and (13) can be rewritten as

$$
v \gg>\frac{v_{d}}{a} \sqrt{\varepsilon_{r}} \max \left\{\sqrt{\varepsilon_{r}}, \sqrt{\varepsilon_{t}}\right\} .
$$

The diffusive regimes can be classified by a particular dependence of the diffusion coefficient, $D$, on the collision frequency $[4,5]$. At sufficiently low collisionality, when the drift frequencies are small,

$$
v_{k}^{\text {eff }} \ll \frac{\dot{\mathrm{r}}_{k}}{\mathrm{a}}, \dot{\theta}_{k},
$$

we obtain the so-called $v$-regime $(D \propto v)$. In the case opposite to $(15)$, we get to $1 / v$ regime $(D \propto 1 / v)$. Since the drift frequency of the bananas is

$$
\frac{\dot{\mathrm{r}}_{\mathrm{f}}}{\mathrm{a}} \approx \frac{\mathrm{v}_{d}}{\mathrm{a}} \sqrt{\frac{\varepsilon_{\mathrm{f}}}{\varepsilon_{\mathrm{r}}}},
$$

the condition (12) for the onset of the regime of the diffusive losses coincides with the condition of the onset of the $1 / \mathrm{V}$-regime. Hence, there is no $\mathrm{v}$-regime for the toroidally trapped particles. 
The situation might be different for the locally trapped particles. Their poloidal drift velocity can be different from $\frac{v_{d}}{a}$,

$$
\dot{\theta}_{r} \approx \max \left\{\Omega_{E}, \frac{v_{d}}{a}, \frac{v_{d}}{\varepsilon_{t}} \frac{\partial \varepsilon_{r}}{\partial r}\right\}
$$

with

$$
\Omega_{E}=\frac{e}{m \Omega} \frac{\partial \Phi}{\partial r} .
$$

Comparing Eqs.(13) and (15), we can conclude that the v-regime exists for the locally trapped particles, provided the condition,

$$
\dot{\theta}_{\mathbf{s}}>\frac{v_{d}}{a},
$$

is fulfilled. This can happen at sufficiently large electric field ( $Ф$ comparable with energy) or when the ripple is larger then the toroidicity.

If the collision frequency obeys the condition opposite to Eq.(14), then the loss is convective and quite rapid. The particles are lost at a rate

$$
\frac{1}{\tau}=\min \left\{\frac{a}{\dot{r}_{1}}, \frac{a}{\dot{r}_{r}}\right\} \text {. }
$$

Almough the motion of the particles, in general, is very complicated, we can imagine the collisionless convective loss as the following. In one of the cases, when bananas drift outwards (Fig.2a), the transition particles start their motion in the locally trapped state and move downwards. As the ripple decreases, they become detrapped and start drifting directly outwards as bananas. In the opposite situation, as bananas drift inwards (Fig.2b), they become locally trapped in the unfavorable part and leave the system as locally trapped particles. The orbit analysis becomes more complicated, if we try to take 
into account all the drifts and the collisionality, different on the different parts of the transition orbit.

\section{Radial displacement of the bananas}

The radial displacement of the toroidally trapped particle on one circuit was evaluated in many papers (see $[3-5,17])$. But the approach used there implied that the ripple was vertically symmetric. In some papers the ripple was considered to be so small, that no wells existed in the reflection points of the banana. Here, we consider the case, when the ripple is strong enough to form the wells and not necessarily vertically symmetric.

The aim is to evaluate the radial displacement of the toroidally trapped particle on one bounce - back and forth- between the reflection points without specifying the particular form of the ripple. To do that, it is convenient to introduce the adiabatic invariant of the banana, $\mathrm{J}($ see $[17])$,

$$
J^{\prime}=2 R_{0} \int_{\phi_{1}}^{\phi_{0}} \frac{B_{0}}{B} \sqrt{2\left\{E-\mu B_{0}\left[1-\varepsilon_{\imath} \cos \left(\beta-\phi^{\prime} / q\right)-\varepsilon_{r} \cos \left(N \phi^{\prime}\right)\right]\right.} d \phi^{\prime},
$$

where

$$
\beta \equiv \theta+\phi / q=\text { constant }
$$

denotes the field line; $\theta$ and $\phi$ being some reference angles through which the line goes. The integrand in Eq.(21) is just the absolute value of the parallel velocity, $u$, multiplied by the length factor $\mathrm{B}_{0} / \mathrm{B}$. The integration is performed between the reflection points (tips of the banana).

The radial displacement of the particle in one circuit is 


$$
d=\oint \frac{d r}{d t} d t
$$

where

$$
\begin{aligned}
& \frac{d r}{d t}=\frac{1}{r \Omega} \frac{u B_{0}}{B} \frac{\partial}{\partial \theta}\left(\frac{u B_{0}}{B}\right), \\
& d t=\frac{d \phi}{\dot{\phi}} \\
& \dot{\phi}=\frac{u B_{0}}{R_{0} B} .
\end{aligned}
$$

This yeilds,

$$
d=\frac{2 R_{0}}{r \Omega} \int_{\phi_{1}}^{\phi_{1}} \frac{\partial}{\partial \theta^{\prime}}\left(\frac{u B_{0}}{B}\right) d \phi^{\prime}=\frac{2 R_{0}}{r \Omega} \int_{\phi_{1}}^{\phi_{2}} \frac{\partial}{\partial \beta}\left(\frac{u B_{0}}{B}\right) d \phi^{\prime} .
$$

Comparing Eqs.(21) and (25), one can see that $\mathrm{d}$ can be evaluated by differentiating $\mathrm{Jt}^{\mathrm{t}}$ with respect to $\beta$ [17]. But in our case, it should be done with caution, because the reflection points, $\phi_{1}$ and $\phi_{2}$, are not smooth functions of $\beta$, but have steps at some $\beta_{1}$ and $\beta_{2}$, corresponding to the situation when the reflection points touch the local maxima (see Fig.3). This steps give rise to delta-functions, when the limits of the integral (21) are being differentiated. In these points $\mathrm{J}$ has a step-like change equal to the separatrix value of the invariant of the locally trapped particle, $\mathrm{J}_{3}^{\mathrm{r}}$,

$$
J_{s}^{z}=R_{0} \oint_{\operatorname{mal}} u \frac{B_{0}}{B} d \phi^{\prime},
$$

where the integration is performed over the local minima near the reflection point (between $\phi_{0}$ and $\phi_{2}$ on Fig.3). Bearing this in mind, for the derivative $\partial J / \partial \beta$ we obtain

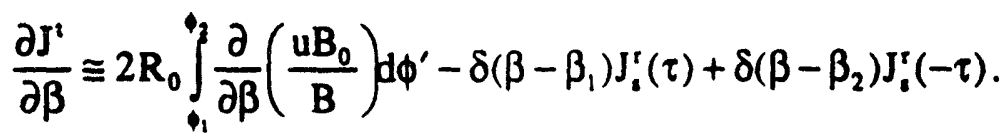




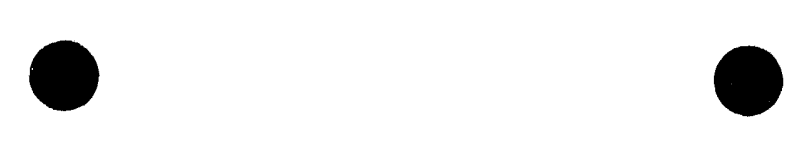

Note, that in Eq.(27) we used the fact that, in accordance with Eq.(22), while one moves along the field line $(\beta=$ constant) with $\phi$ increased, the poloidal angle, $\theta$, decreases.

The adiabatic invariant $\mathfrak{l}$, with the energy and magnetic moment being fixed, is a periodic function of $\beta$, with a period $2 \pi$. Thus, the average of $\partial \mathrm{s} / \partial \beta$ over the period is zero. Averaging Eq.(27) and using Eq.(23) gives the following average radial displacement ,

$$
\bar{d}=\frac{1}{2 \pi} \int_{0}^{2 \pi} d(\beta) d \beta=\frac{N}{2 \pi r \Omega}\left(J_{i}^{\tau}(\tau)-J_{i}^{r}(-\tau)\right)
$$

The factor $\mathrm{N}$ appears, because, while moving along $\beta$, we meet $\mathrm{N}$ local magnetic wells. Note, that the averaging in Eq.(28) is just the averaging over the toroidal phase of the reflection point of the banana. When the ripple does not exist in the upper or lower bounce points, the corresponding $\mathrm{J}_{\mathrm{a}}^{\mathrm{r}}$ in Eq.(28) should be set to zero.

The net radial drift of the banana particles, described by Eq.(28), is independent on $\beta$, i.e. constant on a magnetic surface, is a new phenomena, which was never studied before in the ripple transport studies. It exists only in the vertically asymmetric configurations because the contributions from $\mathrm{J}_{1}^{\mathrm{r}}$ in the upper and lower parts do not compensate each other. In the next section we study the effect of this net drift on transport.

\section{Kinetic equation and diffusive transport}

It is known that the kinetic equation can be written in the divergence form $[18,19]$,

$$
\operatorname{div}_{z}(z|G| F)=|G| C(F)
$$

where $z$ is $2 * N(N=3)$ pseudo-canonical phase space variables with $G$ being the Jacobian of the transformation, $\mathrm{C}(\mathrm{F})$ is the collision operator. In the guiding center theory the 
natural guiding center variables are $\left(y ; E, \mu, \phi_{g}\right)$ with $y$ being the guiding center location, $\mathrm{E}$ the energy, $\mu$ the magnetic moment and $\phi_{g}$ the gyro phase. The Jacobian of these variables is $[18,19]$,

$$
|G|=\frac{B}{u}
$$

To study the transport of thermal particles in the toroidal fusion devices, it is sufficient to use the Lorentz form of the collision operator,

$$
|G| C(F)=v \frac{\partial}{\partial \mu} \mu u \frac{\partial}{\partial \mu} F,
$$

because the distribution function usually has steep changes in narrow regions of the pitch angle, and the slowing down and energy scattering terms can be neglected $[4,5]$.

After the averaging over $\phi_{\mathrm{g}}$, Eq.(29) takes the following form in the toroidal coordinates, $(r, \beta, \phi)$,

$$
\frac{\partial}{\partial r}(\dot{i}|G| F)+\frac{\partial}{\partial \beta}(\dot{\beta}|G| F)+\frac{\partial}{\partial \phi}(\dot{\phi}|G| F)=v \frac{\partial}{\partial \mu} \mu u \frac{\partial F}{\partial \mu},
$$

with the toroidal component of the drift velocity being proportional to the Jacobian (see Eq.(24)).

If the motion of the particles is almost periodic, Eq.(32) can be simplified by averaging over the fast parallel motion $[5,19]$,

$$
\frac{\partial}{\partial r}(\langle\dot{\mathrm{r}}\rangle\langle\mathrm{F}\rangle)+\frac{\partial}{\partial \beta}(\langle\dot{\beta}\rangle\langle\mathrm{F}\rangle)=\frac{\nu}{\mathrm{BT}_{\mathrm{b}}} \frac{\partial}{\partial \mu} \mu \mathrm{I} \frac{\partial}{\partial \mu}\langle\mathrm{F}\rangle+\mathrm{S} .
$$

Here,

$$
\langle\dot{r}\rangle=\frac{1}{T_{b} r \Omega} \oint \frac{u B_{0}}{B} \frac{\partial}{\partial \beta}\left(\frac{u B_{0}}{B}\right) \frac{d \phi}{\dot{\phi}},
$$




$$
\begin{aligned}
& \langle\dot{\beta}\rangle=-\frac{1}{T_{b} r \Omega} \oint \frac{u B_{0}}{B} \frac{\partial}{\partial r}\left(\frac{u B_{0}}{B}\right) \frac{d \phi}{\dot{\phi}}, \\
& \langle F\rangle=\oint F d \phi, \\
& J=\oint R_{0}^{2} \dot{\phi d} \phi=\oint R_{0} \frac{u B_{0}}{B} d \phi, \\
& I=\oint \frac{B R_{0}^{2}}{B_{0}} \dot{\phi} d \phi=R_{0} \oint u d \phi, \\
& T_{b}=\oint \frac{d \phi}{\dot{\phi}}=\frac{\partial J}{\partial E},
\end{aligned}
$$

Here, integration is performed over the period of motion along the field line. We should bear in mind that Eqs.(33)-(38) are different for different types of motion, since the averaged quantities are different for the toroidally and locally trapped particles, although we omitted the distinguishing index for simplicity. Note that, to obtain Eq.(34), we assumed that $(F-<F>)$ is small and, in what follows, we omit the brackets setting $F=\langle F\rangle$. We introduced the quantity I (Eq.(37b)) which is different from the adiabatic invariant, J, and coincides with $\mathrm{J}$ if only we neglect the ripple modulation in the length factor, which is usually implied in most of papers $[4,5,15]$.

In the left hand side of the kinetic equation (33) we inserted the source term S. The appearance of this term is due to the fact that, to perform the bounce averaging, we have to separate the groups of particles with one type of periodic motion from the other. Since there exist collisionless transitions between these groups, we include the source term, $S$, describing these transitions.

Discussion about the radial displacement of the banana in the previous section led us to the following. Due to the fact that the adiabatic invariant of the toroidally trapped particles has steps on the boundary with the locally trapped particles (see Fig.4) the derivatives of $\mathrm{Jt}^{\mathrm{t}}$ have additional terms proportional to delta-functions. That is why the 
averaged velocity of the toroidally trapped particles are not just given by the cross derivatives of the invariant, but also include $\delta$-functions (compare with [19]):

$$
\begin{aligned}
& \langle\dot{r}\rangle^{\prime}=\frac{1}{r \Omega \partial J^{\prime} / \partial E}\left(\frac{\partial J^{\prime}}{\partial \beta}-\delta(Q) \frac{\partial Q}{\partial \beta} J_{c}^{\prime}\right) \equiv \dot{r}^{\prime}+\dot{r}_{b}^{\prime}, \\
& \langle\dot{\beta}\rangle^{\prime}=-\frac{1}{r \Omega \partial J^{\prime} / \partial E}\left(\frac{\partial J^{\prime}}{\partial r}-\delta(Q) \frac{\partial Q}{\partial r} J_{c}^{\prime}\right) \equiv \dot{\beta}^{\prime}+\dot{\beta}_{b}^{\prime},
\end{aligned}
$$

where the condition,

$$
Q(r, \beta, E, \mu)=0 \text {, }
$$

defines the separatrix between the locally and toroidally trapped particles. We imply that $Q>0$ corresponds to the toroidally trapped particles and $Q<0$ to the locally trapped particles. Eqs.(39) and (40) should be compared with Eq.(27). Note that the signs of $\partial Q / \partial \beta$ and $\partial Q / \partial \mathrm{r}$ take care of the different signs of contribution coming from the solutions of Eq.(41) in the different midplanes. In Eqs.(39) and (40) and below, we split the velocities into the main part and the "boundary" part, marked by the subscript " $b "$ "

The adiabatic invariant of the locally trapped particle, $\mathrm{J}$, is defined similarly to $\mathrm{J}$, except that the integration over $\phi$ is performed inside the local magnetic well. We should also keep in mind that $\mathrm{Jr}$ exists only at $\mathrm{Q}<0$, i.e. it also has steps of the size of $\mathrm{Jr}$ itself. This gives rise to similar $\delta$-functions for the derivatives of $\mathrm{Jr}$. The averaged drift velocity of the locally trapped particle can then be presented in the form analogous to Eqs.(39) and (40),

$$
\begin{aligned}
& \langle\dot{\mathrm{r}}\rangle^{r}=\frac{1}{\mathrm{r} \Omega \partial \mathrm{J}^{\mathrm{r}} / \partial \mathrm{E}}\left(\frac{\partial \mathrm{J}^{r}}{\partial \beta}+\delta(Q) \frac{\partial Q}{\partial \beta} \mathrm{J}_{\mathrm{r}}^{\mathrm{r}}\right) \equiv \dot{\mathrm{r}}^{\mathrm{r}}+\dot{\mathrm{r}}_{\mathrm{b}}^{\mathrm{r}}, \\
& \langle\dot{\beta}\rangle^{\mathrm{r}}=-\frac{1}{\mathrm{\Omega} \partial \mathrm{J}^{\mathrm{r}} / \partial \mathrm{E}}\left(\frac{\partial \mathrm{J}^{\mathrm{r}}}{\partial \mathrm{r}}+\delta(Q) \frac{\partial Q}{\partial \mathrm{r}} \mathrm{J}_{\mathrm{r}}^{\mathrm{r}}\right) \equiv \dot{\beta}^{r}+\dot{\beta}_{\mathrm{b}}^{\mathrm{r}} .
\end{aligned}
$$




$$
\langle\dot{r}\rangle^{\prime} \frac{\partial F^{\prime}}{\partial r}+\langle\dot{\beta}\rangle^{\prime} \frac{\partial F^{\prime}}{\partial \beta}+A^{\prime}=\frac{v}{B_{0} T_{b}} \frac{\partial}{\partial \mu} \mu I^{\prime} \frac{\partial F^{\prime}}{\partial \mu}+S^{\prime}
$$

where we introduced the quantity $A^{\prime}$ related to the non-divergence-free part of the velocity, $\dot{r}_{b}^{\prime}$ and $\dot{\beta}_{b}^{\prime}$,

$$
\begin{aligned}
& A^{\prime}=\frac{F^{\prime} \delta(Q)}{r \Omega \partial J^{\prime} / \partial E}\left(\frac{\partial Q}{\partial \beta} \frac{\partial J_{t}^{r}}{\partial r}-\frac{\partial Q}{\partial r} \frac{\partial J_{t}^{r}}{\partial \beta}\right)= \\
& -F^{\prime} \delta(Q)\left(\frac{\partial Q}{\partial \beta} \dot{\beta}^{r}+\frac{\partial Q}{\partial r} \dot{r}^{r}\right)=-\left.F^{t, r} \delta(Q) \frac{d Q}{d t}\right|^{r} .
\end{aligned}
$$

In obtaining Eq.(45) we used the fact that the distribution functions of the locally and toroidally trapped particles are equal on the separatrix.

For further transformation, let us notice that $A^{t}$ turns out to be the velocity of change of the phase volume of the locally trapped region along the drift trajectory of the locally trapped particle. That is why, $A^{t}$ is proportional to the number of collisionless transitions from the locally to toroidally trapped states i.e. is, in fact, equal to the change of the number of toroidally trapped particles per unit of time,

$$
A^{\prime}=-\dot{n}^{r}=\dot{n}^{\prime}=S^{\prime} .
$$

Thus, the part of the kinetic equation related to the divergent part of the velocity is equal to the source, which is not surprising. A similar procedure can be carried out for the kinetic equation of the locally trapped particles, to show that $A^{r}=S^{r}=-A^{t}$, so that the resulting equations take the familiar form $[4,5]$,

$$
\begin{aligned}
& \langle\dot{r}\rangle^{\prime} \frac{\partial F^{\prime}}{\partial r}+\langle\dot{\beta}\rangle^{\prime} \frac{\partial F^{\prime}}{\partial \beta}=\frac{v}{B_{0} \partial J^{\prime} / \partial E} \frac{\partial}{\partial \mu} \mu I^{\prime} \frac{\partial F^{\prime}}{\partial \mu}, \\
& \langle\dot{r}\rangle^{r} \frac{\partial F^{r}}{\partial r}+\langle\dot{\beta}\rangle^{r} \frac{\partial F^{r}}{\partial \beta}=\frac{v}{B_{0} \partial J^{r} / \partial E} \frac{\partial}{\partial \mu} \mu I^{r} \frac{\partial F^{r}}{\partial \mu},
\end{aligned}
$$


where the velocities are given by Eqs.(42),(43),(45),(46) and the distribution functions obey the boundary condition,

$$
\delta(Q) F^{\prime}=\delta(Q) F^{r} \equiv \delta(Q) F^{b}
$$

Let us split the the distribution function into two parts: the average part,

$$
\bar{F}^{\mathrm{i}, \mathrm{r}}=\frac{1}{2 \pi} \oint \mathrm{F}^{\mathrm{i}, \mathrm{r}} \mathrm{d} \beta \text {, }
$$

and the part which varies on the magnetic surface,

$$
\overline{\mathrm{F}}^{\mathrm{t}, \mathrm{s}}=\mathrm{F}^{\mathrm{t}, \mathrm{s}}-\overline{\mathrm{F}}^{\mathrm{t}, \mathrm{s}}
$$

We do this in order to separate the new effect described in the previous section (the $\beta$ independent drift of the toroidally trapped particles) which give rise to the $\beta$-independent addition to the distribution function. The radial fluxes averaged over the magnetic surface,

$$
\Gamma^{i, r}=\oint \frac{d \beta}{2 \pi}\left(\oint \frac{d \phi}{2 \pi} d^{3} v\right)\langle\dot{r}\rangle^{i, r} F^{t, s}
$$

where $\langle\mathrm{r}\rangle^{\mathrm{t}, \mathrm{r}}$ are given by Eqs.(39) and (42), are split correspondingly,

$$
\Gamma^{1, r}=\bar{\Gamma}^{1, r}+\bar{\Gamma}^{1, r}
$$

so that $\bar{\Gamma}^{h, 8}$ and $\tilde{\Gamma}^{h, 8}$ come from $\overline{\mathrm{F}}^{\mathrm{t}, \mathrm{s}}$ and $\tilde{\mathrm{F}}^{\mathrm{l}, \mathrm{r}}$, respectively.

Since the adiabatic invariant is a periodic function of $\beta$, the $\beta$-average of $\partial \mathrm{s}, \mathrm{s} / \partial \beta$ is zero. That is why $\dot{\mathrm{r}}^{1, s}$, proportional to $\partial \mathrm{J} \omega / \partial \beta$ (see Eqs.(39) and (42)), being averaged with $\bar{F}^{i, 8}$, do not contribute to $\bar{\Gamma}^{i, r}$, so that only $\dot{\mathrm{r}}_{b}^{\mathrm{t}, \mathrm{s}}$ are left there. The remaining parts of $\bar{\Gamma}^{t, 8}$ turn out to be equal in the absolute value and have opposite signs for the toroidally and locally trapped particles,

$$
\bar{\Gamma}^{\prime}=-\frac{1}{r R_{0} \Omega} \oint \frac{d \beta}{2 \pi} \int_{\text {log.turap. }} d \mu B_{0} \int d E \delta(Q) \frac{\partial Q}{\partial \beta} J_{2}^{r} \bar{F}^{b}=-\bar{\Gamma}^{r} .
$$


To obtain Eq.(54) we used the equality of the distribution functions of the locally and toroidally trapped particles on the boundary (Eq.(49)), the relation

$$
\oint \frac{d \phi}{2 \pi} d^{3} v=\frac{\partial J^{1,8}}{\partial E} \frac{d E d \mu B_{0}}{R_{0}},
$$

and the fact that, due to the $\delta$-function, the integral over $\mu$ in the toroidally trapped region gives the same as the one over the locally trapped region.

Eq.(54) shows that the fluxes produced by the constant part of the distribution function, $\overline{\mathrm{F}}$, compensate each other, and only $\tilde{F}(\beta)$ contributes to transport. It means, in particular, that the net radial drift of bananas described in the previous section does not result in new transport, its flux being compensated by the corresponding flux of the locally trapped particles.

The contribution to fluxes $\tilde{\Gamma}^{t, 8}$ coming from $\delta$-terms also compensate each other. All that remains, is

$$
\Gamma^{\prime, 8}=\frac{1}{r R_{0} \Omega} \oint \frac{d \beta}{2 \pi} \int_{\text {cos. boc. }} d \mu B_{0} \int d E \frac{\partial J^{i, r}}{\partial \beta} \tilde{F}^{l, r},
$$

that is the diffusion produced by the differences between the phases in the reflection points of the banana, which are zero on average, and the usual diffusion of the locally trapped particles.

The remaining contribution to the diffusion in $1 / \mathrm{N}$-regime of the toroidally trapped particles coming from $\bar{F}^{\prime}$ is almost the same for the up-down symmetric and asymmetric tori. It has been investigated before (see Eq.A.I.4 of [5]) and is related to $1 / \mathrm{N}$-regime of the locally trapped particles by the relation, $D_{t}^{\text {orm }} / D_{f}^{\text {orm }}=\frac{1}{(q N)^{2}} \sqrt{\frac{\varepsilon_{q}}{\varepsilon_{\mathrm{g}}}}$. Although, note that some new effects due to the asymmetry, which do not change the order of the fluxes, can be expected in the results obtained in [4,5] since we should include the general (up-down 
asymmetric) dependence of $\varepsilon_{\mathrm{r}}$ on the poloidal angle. It will give new terms for $\frac{\partial \mathrm{J}^{\mathrm{t}, \mathrm{t}}}{\partial \beta}$ with a relative order $\frac{\partial \varepsilon_{\mathrm{r}} / \partial \beta}{\varepsilon_{\mathrm{t}}}$ in comparison with the conventional ones.

We find the effect of compensation of the diffusive fluxes quite curious. It is a consequence of Liouville's theorem, which turns out to work even for the collisional case, because the effects investigated here are independent on the magnetic field line of the magnetic surface. Substantial new effects due to the vertical asymmetry should occur in the convective transport, when the deviation of the distribution function from the Maxwellian can not considered a perturbation. In this case the kinetic equations (50) and (51) are still valid, but need an introduction of a loss cone. This approach was developed in [19] and can be carried out analytically only for the simplest case of the vertically homogeneous ripple without electric field.

\section{Explicit evaluation of $\overline{\mathbf{F}}$}

Although the flux of the toroidally trapped particles due to up-down asyrismetry is compensated by the opposite flux of the locally trapped particles, it is interesting to evaluate it. We perform this procedure below, implying that $\varepsilon_{\mathrm{r}} / \varepsilon_{\mathrm{t}} \ll<1$, which allows us to set $I^{t, r}=J^{t, r}$ (given by Eqs.(37a) and (37b)) in Eqs.(47) and (48). Kinetic equation for $\bar{F}^{\prime}$ in the main order takes the form (compare with Eq.(47)),

$$
\bar{d} \frac{\partial \bar{F}^{\prime}}{\partial \tau}=\frac{v}{B_{0}} \frac{\partial}{\partial \mu} \mu^{\prime} \frac{\partial \bar{F}^{\prime}}{\partial \mu},
$$

where we took into account that the radial velocity of the banana $<\dot{r}>^{\prime}$ (Eq.(39)) averaged over $\beta$ is equal to $\frac{\bar{d}}{\partial J^{\prime} / \partial E}$ with $\bar{d}$ given by Eq.(28). For further convenience, let us transform from $\mu$ to the variable $k$, 
where variable $\tau$ is defined to be the reflection point of the toroidally trapped particle in the upper part, when $\varepsilon_{r}=0$,

$$
1-\varepsilon_{\imath} \cos (\tau)=\frac{E}{\mu B_{0}},
$$

$0 \leq \tau \leq \pi$

Then Eq.(57) takes the form,

$$
\overline{\mathrm{d}} \frac{\partial \mathrm{F}_{\mathrm{m}}}{\partial \mathrm{r}}=\frac{v}{\mu \mathrm{B}_{0} \varepsilon_{\mathrm{r}}^{2}} \frac{\partial}{\partial \kappa} \mathrm{J}^{\prime} \frac{\partial}{\partial \kappa} \Psi,
$$

where we expanded the distribution function in the parameter $\frac{v_{d}}{a v_{t}^{e f}}$, so that $\bar{F}^{\prime}=F_{m}+\Psi$ with $F_{m}$ the Maxwellian and $\Psi$ being the first order correction. The expansion parameter is small indeed, since in the diffusive regime condition (12) is fulfilled. The solution can be found straightforwardly,

$$
\Psi=\frac{\partial F_{\mathrm{m}}}{\partial \mathrm{r}} \frac{\mu \mathrm{B}_{0} \varepsilon_{1}^{2}}{v} \int_{-1}^{x} \frac{\mathrm{d} \kappa^{\prime}}{\mathrm{J}^{\prime}\left(\boldsymbol{K}^{\prime}\right)} \int_{1}^{x^{\prime}} \overline{\mathrm{d}}\left(\boldsymbol{K}^{\prime \prime}\right) \mathrm{d} \kappa^{\prime \prime} .
$$

To choose the integration limits in Eq.(61), we used the following boundary conditions:

$$
\begin{aligned}
& \left|\frac{\partial \Psi}{\partial K}(\kappa=1)\right|<\infty, \\
& \Psi(\kappa=-1)=0,
\end{aligned}
$$

at the boundary with the passing particles $(K=-1)$ and for the deeply trapped particles $(K=1)$. The flux from $\Psi$ is

$$
\bar{\Gamma}^{\prime}=\oint \frac{d \beta}{2 \pi}\left(\oint \frac{d \phi}{2 \pi} d^{3} v\right) \frac{\bar{d}}{\partial J^{\prime} / \partial E} \Psi
$$

Let us simplify the calculation by assuming that the ripple exists only in one of the parts of the torus and does not depend on the poloidal angle, so that 


$$
\begin{aligned}
& |\bar{d}|=\frac{8 R \sqrt{\mu B_{0} \varepsilon_{z}}}{\pi \Omega} \\
& \mathrm{J}_{\mathrm{s}}^{\mathrm{r}}=\frac{16 \mathrm{R}}{\mathrm{M}} \sqrt{\mu \mathrm{B}_{0} \varepsilon_{\mathrm{r}}} . \\
& \mathrm{J}^{\prime}=16 \mathrm{qR} \sqrt{\mu \mathrm{B}_{0} \varepsilon_{\mathrm{t}}} \mathrm{f}(\tau) \\
& f(\tau)=E\left(\sin ^{2}(\tau / 2)\right)-\cos ^{2}(\tau / 2) K\left(\sin ^{2}(\tau / 2)\right) \approx \frac{1-\kappa}{2},
\end{aligned}
$$

into Eq.(61), then Eq.(65) into (64), yields

$$
\bar{\Gamma}^{\prime}=-\frac{8 \varepsilon_{t}^{5 / 2} \varepsilon_{\mathrm{r}}}{(\pi \Omega)^{2}} \int \mathrm{dE} \frac{\mathrm{E}^{5 / 2}}{\mathrm{v}(\mathrm{E})} \frac{\partial \mathrm{F}_{\mathrm{m}}}{\partial r} \mathrm{I}
$$

where

$$
I=-\oint \frac{d \theta^{\infty}}{\pi} \int_{-1}^{\infty e(\theta)} d K \int_{-1}^{x} \frac{d K^{\prime}}{\left(1-K^{\prime}\right)} \int_{1}^{x^{\prime}} d K^{\prime \prime}=2
$$

Note, that Eq.(68) is obtained in the limit $\alpha \rightarrow 0, \varepsilon_{\mathrm{r}} / \varepsilon_{\mathrm{t}} \rightarrow 0$. The diffusion coefficient corresponding to this flux, $D_{t}^{\infty} \propto \varepsilon_{\mathrm{r}} \sqrt{\varepsilon_{\mathrm{t}}} \frac{v_{d}^{2}}{v}$, could be found from the simple heuristic

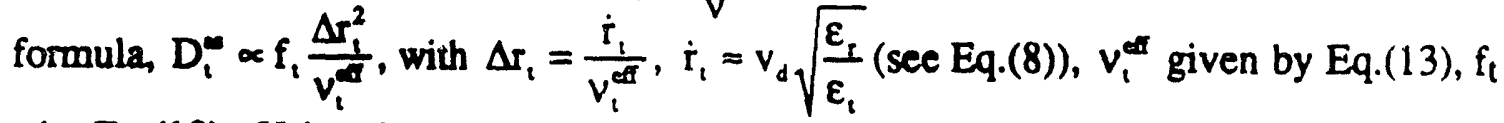
- by Eq.(10). Using the same heuristic formula for the usual (up-down symmetric) $1 / v$ regime of the locally trapped particles, $D_{s}^{s y m} \propto f_{r} \frac{\Delta r_{r}^{2}}{v_{r}^{s e}}$, with $\Delta r_{r}=\frac{v_{d}}{v_{r}^{d t}}, f_{r}$ given by Eq.(11), we obtain the well known [5] result (correct for $\alpha<1$ ), $D_{r}^{\text {ind }} \propto \varepsilon_{r}^{3 / 2} \frac{v_{d}^{2}}{v}$. The value of the ratio of these diffusion coefficients, $D_{t}^{\infty} / D_{r}^{\infty-m} \approx \sqrt{\frac{\varepsilon_{t}}{\varepsilon_{t}}}$, was what motivated us to investigate this diffusive regime. But, as we have shown above, the asymmetric flux of the toroidally trapped particles, originated from their net radial drift, independent on $\beta$, is compensated in the main order by the additional flux of the locally trapped particles. This 
can be explicitly shown, if we put $\Psi$ given by Eq.(61) into the formula for the asymmetric flux of the locally trapped particles,

$$
\bar{\Gamma}^{r}=\oint \frac{d \beta}{2 \pi}\left(\oint \frac{d \phi}{2 \pi} d^{3} v\right) \frac{\partial J^{r} / \partial \beta}{\partial J^{r} / \partial E} \Psi_{b}=-\bar{\Gamma}^{\prime},
$$

where $\Psi_{b}$ is the distibution function $\Psi$ on the boundary with the locally trapped particles $(K=\cos (\theta))$.

Note, that explicit investigation of the case $\varepsilon_{\mathrm{r}} / \varepsilon_{\mathrm{t}} \approx 1$ is more complicated. We do not perform that, because the general result about compensation of the fluxes arising from $\overline{\mathrm{F}}$ is valid for arbitrary relationship between $\varepsilon_{\mathrm{t}}$ and $\varepsilon_{\mathrm{r}}$.

\section{Discussion of the results}

The phenomena investigated above are a new kind of transport processes which arises due to the ripple asymmetry in upper and lower parts of the torus. This asymmetry can occur due to the vertical displacement of the plasma or the vertical asymmetry of the magnetic configuration (like in the case of the single null divertor). The change of the ripple magnitude can be as high as unity for the tokamak. Similar phenomena can occur in the stellarator. The resulting equations for the radial displacement and the radial flux are applicable both for the stellarator and tokamak.

It is shown that the vertical asymmetry gives rise to a net radial drift of the

toroidally trapped particles with the characteristic velocity $\frac{v_{d}}{\sqrt{\varepsilon_{t}}}\left(\sqrt{\varepsilon_{r}^{+}}-\sqrt{\varepsilon_{r}^{-}}\right)$, where $\varepsilon_{r}^{+}$and $\varepsilon_{s}^{-}$are the ripple magnitudes in the upper and lower parts of the torus. At low collisionality, the losses of the particles have a convective character. The particles rapidly leave the device either in the toroidally trapped state, when they acquire the net radial outward drift, or in the locally trapped state after experiencing entrapment in the 
unfavorable part of the system. The new mechanism presents a new channel of the particle loss, so that for every device there exists a critical value of the allowed vertical asymmetry, which needs to be estimated in each particular case.

In the high collisionality regime, the diffusion of the toroidally trapped particles associated with the asymmetry is shown to be compensated by the additional diffusive flux of the locally trapped particles. This is related to the fact that a distribution function constant on a magnetic surfaces does not contribute to transport. That is the reason why the net radial drift of bananas - the dominant part of which does not vary on a magnetic surface - does not change the transport. The remaining part of the radial drift velocity of the bananas is a rapid function of the poloidal angle (or $\beta$ ) and its contribution to the fluxes have been investigated in $[4,5]$. 


\section{Figure captions:}

Fig. 1. Contour lines, $\varepsilon_{r}=$ constant, for the vertically shifted plasma. The picture is obtained numerically (courtesy of Azita Valinia) for TEXT-U, plasma has been moved down by $2.5 \mathrm{~cm}$.

Fig.2a. The bounce-averaged transition orbit for the case when the ripple magnitude is higher in the upper part of the torus: " $r$ " corresponds to the locally trapped part, $t "$ - to the toroidally trapped of the orbit. The particle tends to detrap in the lower part and drifts outwards in the toroidally trapped state due to asymmetry of the reflection points.

Fig.2b. The bounce-averaged transition orbit for the case when the ripple magnitude is higher in the lower part of the torus: " $r$ " corresponds to the locally trapped part, " $t$ " - to the toroidally trapped of the orbit. The particle tends to entrap in the lower part and drifts inwards in the toroidally trapped state due to asymmetry of the reflection points.

Fig.3. Illustration to Eqs.(23), (24). When the toroidal phase of reflection corresponds to "touching" of the magnetic maxima, the adiabatic invariant $\mathrm{J}$ increases by amount $\mathrm{J}$, equal to the separatrix value of the invariant of the locally trapped particle.

Fig.4. The schematic depiction of the longitudinal motion of the particles with the same magnetic moment, $\mu$, on the plane $(\phi, \theta)$. Straight lines correspond to different values of $\beta$, marking different field lines. At some particular values of $\beta, \beta_{i}$ and $\beta_{j}$, the adiabatic invariant $\mathrm{Jt}$ changes abruptly by amount $\mathrm{Jr}$. The "total" invariant J, obtained from all the pieces along the field line, is free from these abrupt changes. 


\section{References:}

1. H.P. Furth, R.J. Goldston, S.J. Zweben, D.J. Sigmar, Nuclear Fusion 30, 1799 (1990).

2. R.B. White, H.E. Mynick, Phys. Fluids B 1, 980 (1989).

3. R.J. Goldston, R.B. White, A.H. Boozer, Phys. Rev. Let. 47, 647 (1981).

4. P.N. Yushmanov, in Reviews of Plasma Physics, edited by B.B.Kadomtsev (Consultants Bureau, New York, 1991), Vol.16, p.117.

5. L.M.Kovrizhnykh, Nuclear Fusion 24, 851 (1984).

6. K. Tani, T. Takizuka, M. Azumi, H. Kishimoto, Nuclear Fusion 23, 657 (1983).

7. L.M. Hively, Nuclear Fusion 24, 779 (1984).

8. F.S.Zajtsev, A.P.Smimov, P.N.Yushmanov, Nuclear Fusion 26, 1311 (1986).

9. R.B. White, A.H. Boozer, R.J. Goldston, R. Hay, J. Albert, C.F.F. Karney, in Plasma Physics and Controlled Nuclear Fusion Research, 1982, Proc. of the 9th International Conference (IAEA, Vienna, 1983), Vol.III, p.391.

10. K. Tani, T. Takizuka, M. Azumi, in Proceedings of the Workshop on 'Statistical Plasma Physics,' US-Japan Joint Institute for Fusion Theory Program (Institute of Plasma Physics), Nagoya 16, 199 (1976).

11. G. Sadler, P. Barabaschi, E. Bertolini e.a., in Proceedings of the 19th International Conference on Plasma Physics, Insbruck 16C, Part I, 167 (1992). 
12. C.D. Beidler, W.N.G. Hitchon, J.L. Shohet, J.Comput.Phys. 72, 220 (1987).

13. D.P. Schlissel, T.H. Osborne, J.C. DeBoo, J.R. Ferron, E.A. Lazarus, T.S. Taylor, Nuclear Fusion 32, 689 (1992).

14. R.B. Bengston, A. Ouroua, A.G. Meigs, Bulletin of the American Physical Society, 34, No 9, paper $8 S 3$.

15. L.M. Kovrizhnykh, S.G. Shasharina, Nuclear Fusion 30, 453 (1990).

16. John R. Cary, S.G. Shasharina, submitted to Phys.Fluids.

17. B.B. Kadomtsev, in Plasma Phys. and the Problem of Controlled Thermonuclear Reactors, ed. MA. Leontovich (Izd. Akad.Nauk SSSR,1958) v.3, p.285-299.

18. R.G. Littlejohn, J.Plasma Physics 29, 111 (1983).

19. A.V. Gurevich, Ya.S. Dimant, in Reviews of Plasma Physics, edited by B.B.Kadomtsev (Consultants Bureau, New York, 1991), Vol.16, p.1. 




\section{Region Stop Erase Close}

\section{Ripple Map \#0}

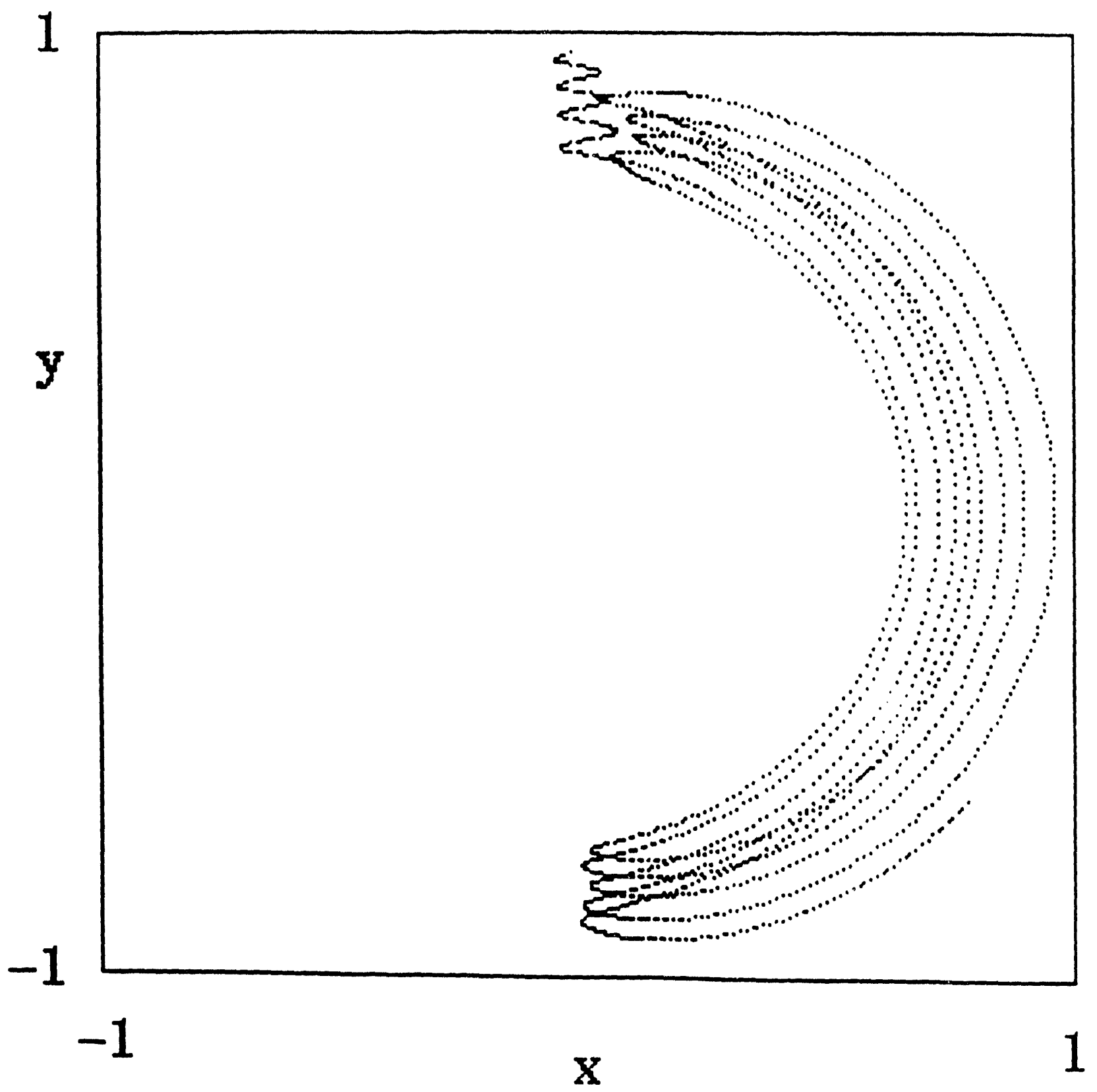




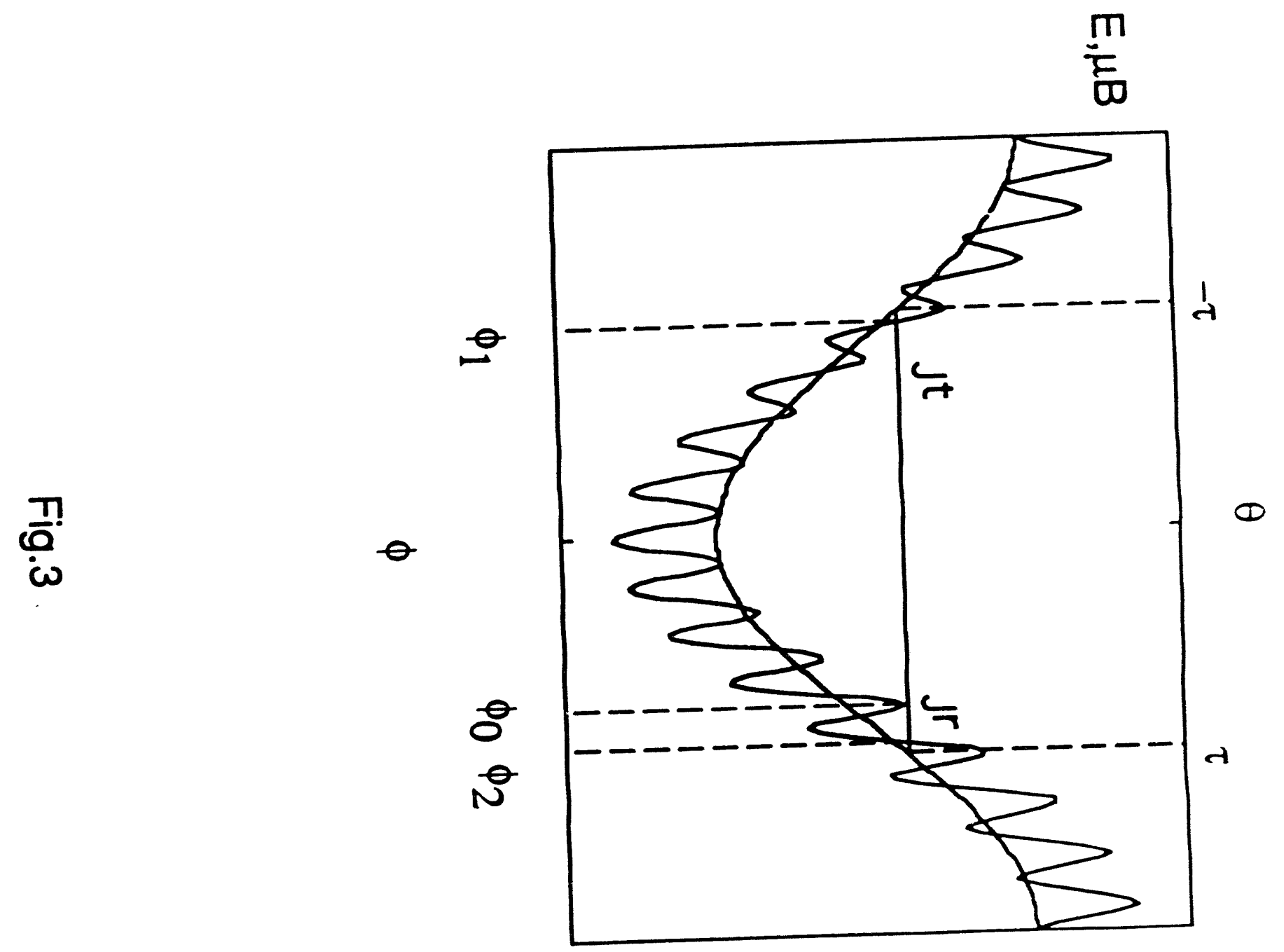




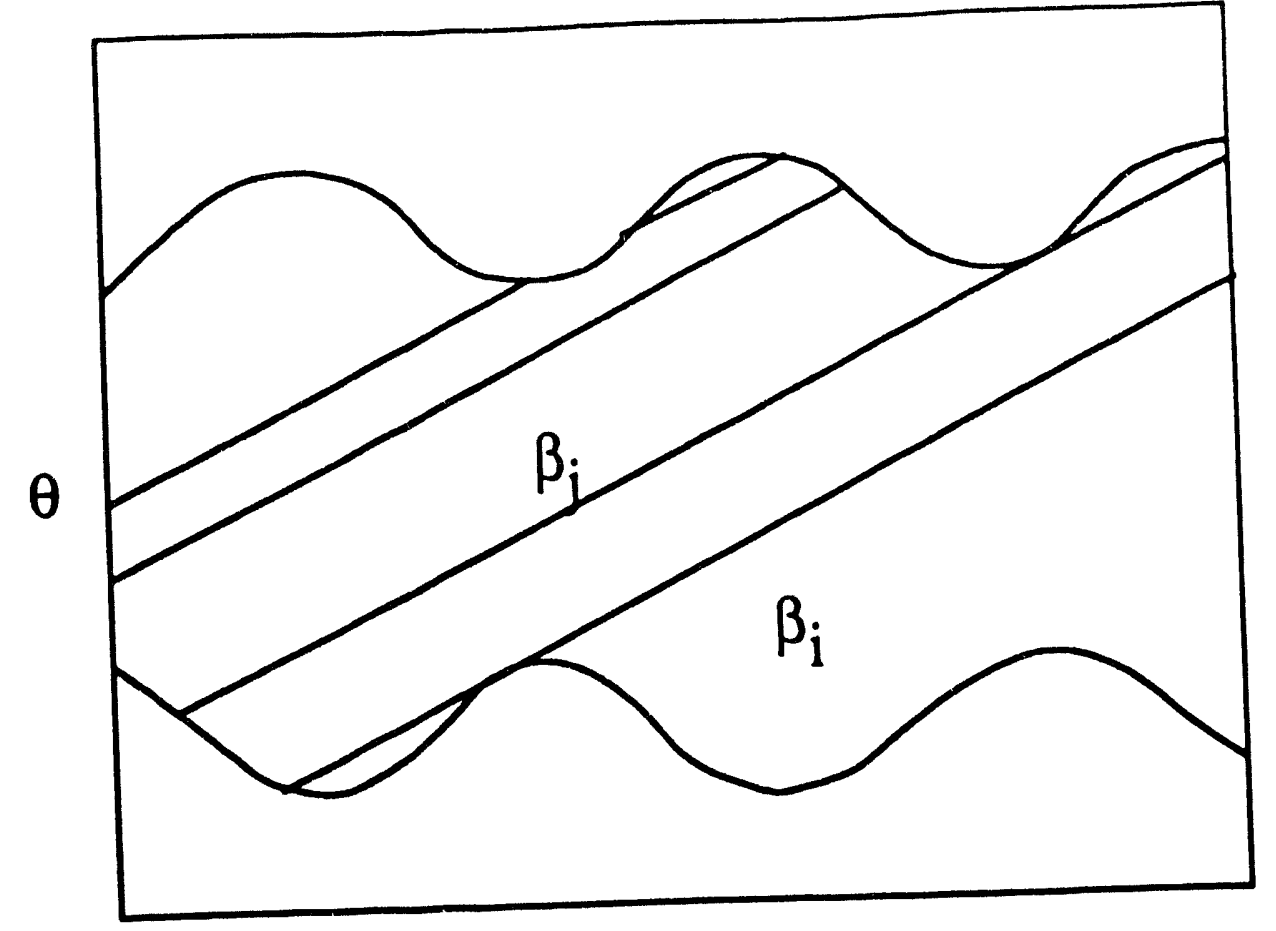

$\phi$

Fig.4 


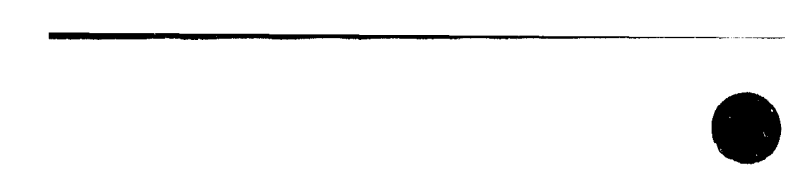

Appendix D. Bootstrap Cuurent of Transition Particles in NonAxisymmetric Tori 


\title{
Bootstrap Current of Transition Particles
}

\author{
S.G.Shasharina, ${ }^{*}$ P.N.Yushmanov, ${ }^{* *}$ John R.Cary,
}

\begin{abstract}
Transition particles make transitions back and forth between the toroidally and locally trapped states. The transition probability gives the relative rate of toroidally trapped particles to become locally trapped. For high energy particles or in the case of the electric potential comparable with the temperature of bulk plasma, the transition probability can be quite large, i.e. close to unity. The transition orbits then consist of one branch corresponding to the locally trapped state and another, on which the particle is toroidally trapped. Since in the locally trapped state the particle moves almost vertically, meanwhile it always moves in some particular toroidal direction in the toroidally trapped state, such orbits should modify the longitudinal transport, bootstrap current in particular. In our work we evaluate the bootstrap current corresponding to such transition particles.
\end{abstract}

** University of Colorado, Boulder, CO 80309-0391

** Institute for Fusion Studies and Fusion Research Center, The University of Texas at Austin, Austin, Texas 78712 


\section{Introduction}

Bootstrap or "diffusion-driven" current is an interesting phenomenon predicted by the neoclassical transport theory $[1,2]$. The phenomenological explanation of its nature is proposed in [3]. Basically, any parallel current in the toroidal devices, sustained without parallel electric field, is the bootstrap current [4]. It exists because of certain asymmetry of the distribution function for the particles moving in parallel and anti parallel direction. The latter is caused by Jifferent radial displacement of those particles, due to which they "feel" different conditions, since the temperature and density generally are non-uniform.

The bootstrap current has been evaluated in many papers. Thus, the investigation of the axisymmetric case is performed in $[2,4-7]$. Studies performed for nonaxisymmetric configurations deal, though, with the helically symmetric magnetic field (a straight stellarator), which reduces the problem to the axisymmetric case [4] with the corresponding changes in the parameters. In that approach only the locally trapped and passing particles exist. Since the toroidicity is neglected, such important phenomenon as collisionless trapping of the toroidally trapped particles into local wells can not be taken into account.

In the present paper, we are going to study the effect produced by the trap-detrap processes on the bootstrap current, i.e. we evaluate the parallel current of the transition particles. The transition orbits consist of the toroidally trapped and the locally trapped branches. Since in the locally trapped state the particle practically does not move in the parallel direction, there is an obvious asymmetry of number of particles moving in parallel and antiparallel directions This asymmetry should be proportional to the trapping probability [8] which presents the fraction of the toroidally trapped particles trapped in the reflection point. In fact, the larger the trapping probability is, the less is the number of the reflected toroidally trapped particles, the larger is the difference between the 
number of the particles moving in different directions. Since the bootstrap current is proportional to the radial width of the orbit, at low collisionality it should be proportional to the width of the transition orbit, which is determined by the locally trapped branch and is much larger then the displacement of non-transitional toroidally trapped particle. All these considerations motivated our studies.

\section{Trapping probability}

The magnetic field with helical perturbation in a stellarator (or ripple in a tokamak) has the form,

$$
B=B_{0}\left[1-\varepsilon_{\mathrm{t}} \cos (\theta)-\varepsilon_{\mathrm{r}} \cos (\mathrm{N} \phi-l \theta)\right],
$$

where $\varepsilon_{\mathrm{t}}=\mathrm{r} / \mathrm{R}_{0}$ is the inverse aspect ratio (toroidicity), $\varepsilon_{\mathrm{r}}$ is the ripple magnitude (or the helical modulation, for the stellarator); $l$ and $\mathrm{N}$ (usually $\mathrm{N} \gg>l$ ) are the poloidal and toroidal mode numbers for a stellarator, while $l=0$ for number for a tokamak; $\theta$ and $\phi$ are the poloidal and toroidal angles, which are related to each other on the magnetic field lines via the rotational transform, $d \phi / d \theta=-1 / t=-q ; r$ and $R=R_{0}\left(1+\varepsilon_{t} \cos (\theta)\right)$ are the minor and major radius.

In such field there exist basically three types of particles: the passing, toroidally and locally trapped. This separation becomes relative as we take the drift motion into account, due to which the toroidally trapped particles might become the locally trapped and vice versa. This transition process depends on the geometrical parameters of the magnetic field, its magnitude and the relative value of the radial electric field, which determine the role of the drift motion and, the transition probability. Why we need to introduce the probability in the deterministic picture of motion? The reason for that is we can not keep track of all the degrees of freedom in the 3-dimensional system and perform the averaging $[\mathrm{KS}, \mathrm{CS}$ ] over the fast toroidal phase of motion, $\phi$. Since the exact behavior of particles near transition points is determined by the toroidal phase, we can 
predict only the average relative number of reflections and transitions at the reflection point of the toroidally trapped particle. The fraction of transitions is called a transition probability $[\mathrm{C}, \mathrm{CS}]$.

Let us introduce some terminology $[\mathrm{C}, \mathrm{CS}]$. We distinguish between positively and negatively moving toroidally trapped particles and call them respectively a minus or plus state ("-" and "+"). We mark the locally trapped state with the index " $r$ ". We call a state majority state if from this state a branching of the trajectory is possible.

The transition probability is determined by the relative change of the phase volumes corresponding to different states. This change occur due to the drift motion. Since the total volume or the phase is conserved, the drift changes of the volumes are related via

$$
\dot{\mathrm{Y}}_{+}+\dot{\mathrm{Y}}_{-}+\dot{\mathrm{Y}}_{\mathrm{r}}=0
$$

Here, $Y_{+,-, r}$ are the phase volume corresponding to the,,+- and $r-s t a t e s$. The time derivative in Eq.(2) implies the derivative along the drift trajectory,

$$
\frac{\mathrm{d}}{\mathrm{dt}}=\dot{\mathrm{r}}_{\mathrm{d}} \frac{\partial}{\partial \mathrm{r}}+\dot{\theta}_{\mathrm{d}} \frac{\partial}{\partial \theta}
$$

with $\dot{\mathrm{r}}_{d}$ and $\dot{\theta}_{d}$ being the drift velocities in the radial and poloidal directions.

Since the number of particles in some particular state is proportional to the phase volume, we can talk of trapping only when

$$
\dot{\mathrm{Y}}_{+}>0 \text {. }
$$

When it is combined by,

$$
\dot{\mathrm{Y}}_{+} \dot{\mathrm{Y}}_{-}<0
$$


the majority state is + or - , namely that which has $\dot{\mathrm{Y}}_{\mathrm{mjs}}<0$ (here mjs $=+$ or - ). The transition orbit corresponding to Eqs.(4) and (5) and with the negative state being the majority is depicted on Fig.1. The conditions (4) and (5) corresponds to the trapping point " $T$ ". In the symmetrical point "S" all $\dot{Y}_{i}(i=+,-, r)$ change their sign. The trapping probability is defined in this case as

$$
P=-\frac{\dot{Y}_{r}}{\dot{Y}_{m j z}}
$$

and refers to the trapping point "T". In accordance with Eqs.(2), (4) and (5) the probability defined by Eq.(6) obeys

$$
0<\mathrm{P}<1 \text {. }
$$

When Eq.(4) is combined by

$$
\dot{\mathrm{Y}}_{+} \dot{\mathrm{Y}}_{-}>0
$$

which is obviously implies that $\dot{Y}_{+}, \dot{Y}_{-}<0$, the ripple state is the majority state and the orbit looks like is shown on Fig.2. This type of orbit is possible only in the case of very strong drift, so that in the poloidal cross-section both + and - states drift in one direction, transforming into r-state in the point "T". In "S" point all $\dot{Y}_{i}(i=+,-, r)$ have opposite signs. The trapping probability given by Eq.(6) does not make sense in this case, since it will give the values of $\mathrm{P}$ larger then unity. But we can introduce another probabilities, referring to the point "S": the probability for $r \rightarrow+$ and $r \rightarrow-$ transitions:

$$
\begin{aligned}
& \mathrm{P}_{+}=-\frac{\dot{\mathrm{Y}}_{+}}{\dot{\mathrm{Y}}_{\mathrm{r}}} \\
& \mathrm{P}_{-}=-\frac{\dot{\mathrm{Y}}_{-}}{\dot{\mathrm{Y}}_{\mathrm{r}}}=1-\mathrm{P}_{+}
\end{aligned}
$$

Since the case described by Eqs.(4) and (8) is quite rare, we shall consider the previous one, assuming that the majority state is + or - . But we still include the possibility of 
large $P$, i.e. close or equal to unity (which is possible when instead of inequality (5) we have equality and $\dot{\mathrm{Y}}_{\mathrm{mj \textrm {s }}}=-1=-\dot{\mathrm{Y}}_{\mathrm{r}}$ ).

The evaluation of the trapping probability is straightforward if the quantities $\dot{\mathrm{Y}}_{+}, \dot{\mathrm{Y}}_{-}$and $\dot{\mathrm{Y}}_{\mathrm{r}}$ are known as functions of coordinates. Since these functions are equal to the values of the adiabatic invariants on the separatrices, we can use the well known expressions for the invariants of the locally and toroidally trapped particles. The lowest order approximation for them gives (see [CS])

$$
P=\frac{f}{1+f / 2}
$$

where $\mathrm{f}$ in the lowest order in $\varepsilon_{\mathrm{r}}, \varepsilon_{\mathrm{t}}$ and $\alpha=\frac{\varepsilon_{\mathrm{t}}}{\mathrm{qN} \varepsilon_{\mathrm{r}}}$ is

$$
\mathrm{f}=\frac{8 \mathrm{q} \sqrt{\mu \mathrm{B}_{0} \varepsilon_{\mathrm{r}}}}{\pi \varepsilon_{\mathrm{r}} \Omega}\left(\frac{\mathrm{V}^{\prime}}{\mu \mathrm{B}_{0}}+\frac{\varepsilon_{\mathrm{r}}^{\prime}}{2 \varepsilon_{\mathrm{r}}}\right)
$$

Here, $\Omega=\frac{e B_{0}}{m c}$ is the gyrofrequency, $V=e \Phi / m, \Phi$ is the electric potential, $(\ldots)^{\prime} \equiv \frac{\partial}{\partial \mathrm{r}}$. The quantity $f$ is a ratio of the flux of the particles getting trapped to the unperturbed incoming flux of the toroidally trapped particles. On Fig.3a the flux of trapping particles is limited by the poirits $A$ and $B$. The unperturbed (i.e. neglecting the drift) flux, $F_{0}$, of the toroidally trapped particles is limited by points $A$ and $C$. Then the relative perturbed flux of the toroidally trapped particles is $1+f / 2$, it is limited by $A$ and $D$. The points $B$ mark the trajectory which gets on the lower maximum of the well after one bounce, so that all the trajectories between $A$ and $B$ become locally trapped. The point $D$ marks the toroidally trapped trajectory which comes on the higher maximum of the well (see Fig. $3 b)$, so that all the trajectories between $A$ and $D$ represent the incident flux of the majority particles. Since from $(1+\mathrm{f} / 2) \mathrm{F}_{0}$ of the incident particles $\mathrm{fF}_{0}$ particles become locally trapped, only $(1-\mathrm{f} / 2) \mathrm{F}_{0}$ get reflected. This is the origin of the asymmetry producing the bootstrap current of the transition particles. 
Keeping this asymmetry in mind, we generalize the well known formula for the integrating of some arbitrary function $G$ over the toroidally trapped state in the velocity space and averaging over the magnetic surface, which is correct when the numbers of + and - states are equal,

$$
\oiint \frac{d \theta d \phi}{(2 \pi)^{2}} \int d^{3} v G(v)=\oiint \frac{d \theta d \phi}{2 \pi} \int d E \int_{0}^{\frac{E}{\left(1+\varepsilon_{0}\right) B_{0}}} \frac{d \mu B}{|u|}(G(\sigma=-1)+G(\sigma=+1)),
$$

to the formula taking into account trapping effects,

$$
\begin{aligned}
& \oiint \frac{d \theta d \phi}{(2 \pi)^{2}} \int d^{3} v G(v)= \\
& \oiint \frac{d \theta d \phi}{2 \pi} \int d E \int_{0}^{\frac{E}{\left(1+e_{1}\right) B_{0}}} \frac{d \mu B}{|u|}\left[\left(1+\frac{f}{2}\right) G(\sigma=m j s)+\left(1-\frac{f}{2}\right) G(\sigma=-m j s)\right] .
\end{aligned}
$$

Here, $\sigma=\operatorname{sign}(u), m j s=+$ or,$- u$ is the parallel velocity, $u=\sigma \sqrt{2\{E-\mu B\}}$. In particular case, when

$$
\mathrm{G}=\mathrm{uF} \text {, }
$$

and $\mathrm{F}$ is a symmetric function, which does not depend on $\phi$,

$$
\mathrm{F}(\sigma=1)=\mathrm{F}(\sigma=-1),
$$

Eq.(14) yields,

$$
\operatorname{mjs} \oint d \theta \int d E \int_{0}^{\frac{E}{\left(1+\varepsilon_{1}\right) B_{0}}} d \mu B f(\mu, E, \theta) F(\mu, E, \theta), \quad \text { mjs }=1,-1 .
$$

Note, that in Eq.(17) we allow $f$ to be dependent on the poloidal angle, which it is, if the higher order formula for $f$ is used rather then the lowest order approximation given by Eq.(12) [CS]. 
3. Solution of the kinetic equation for the transition particle at low-collisional regime.

In order to find the bootstrap current, we need to solve the bounce averaged kinetic equation,

$$
\dot{\mathrm{r}} \frac{\partial \mathrm{F}}{\partial \mathrm{r}}+\dot{\theta} \frac{\partial \mathrm{F}}{\partial \theta}=\frac{v}{\mathrm{~B}_{0} \frac{\partial J}{\partial E}} \frac{\partial}{\partial \mu} \mu \tilde{\mathrm{J}} \frac{\partial \mathrm{F}}{\partial \mu}
$$

where $\dot{\mathrm{r}}$ and $\dot{\theta}$ are the bounce averaged radial and poloidal drift velocities,

$$
\begin{aligned}
& \dot{\mathrm{r}}=\frac{1}{\mathrm{r} \Omega} \frac{\partial \mathrm{J} / \partial \theta}{\partial \mathrm{J} / \partial \mathrm{E}}, \\
& \dot{\theta}=-\frac{1}{\mathrm{r} \Omega} \frac{\partial \mathrm{J} / \partial \mathrm{r}}{\partial \mathrm{J} / \partial \mathrm{E}},
\end{aligned}
$$

$J$ is the adiabatic invariant. It has different forms for the locally $\left(" r ": k^{2} \leq 1\right)$ and toroidally ("t": $\left.k^{2} \geq 1\right)$ trapped particles,

$$
\begin{aligned}
& J^{r}=R_{0} \oint p_{\varphi} d \phi=2 R_{0} \int_{\phi_{k}}^{\phi_{k+1}} \frac{|u| B_{0}}{B} d \phi, \\
& J^{t}=2 R_{0} \int_{\phi_{k+1}}^{\phi_{k}} p_{\phi} d \phi=2 R_{0} \int_{\phi_{k}+1}^{|l u| B_{0}} \frac{B}{B} d \phi+\sigma I^{\prime} . \\
& I^{\prime}=\frac{4 \pi}{N} \frac{\Omega R}{B_{0}} A_{l},
\end{aligned}
$$

Here,

$$
\mathrm{p}_{\varphi}=\frac{\mathrm{uB}}{\mathrm{B}}+\frac{\mathrm{e}}{\mathrm{mc}} \mathrm{A}_{\varphi}
$$

is the momentum conjugated to $\phi, A_{\phi}$ is the toroidal component of the vector-potential. Integration in Eqs.(21) and (22) is performed over one period of motion, $\phi_{\mathbf{k}}$ and $\phi_{\mathbf{k}+1}$ are the toroidal coordinates of the adjacent magnetic maxima. The quantity $\tilde{\mathrm{J}}$ in Eq.(18) differs from the adiabatic invariant, 


$$
\tilde{J} \equiv 2 R_{0} \int_{\phi_{k}}^{\operatorname{rut}} u d \phi
$$

At $\alpha<<\varepsilon_{\mathrm{b}} \varepsilon_{\mathrm{r}}<<\varepsilon_{\mathrm{t}}$, the quantities $\mathrm{Jr}, \mathrm{J}$, $\tilde{\mathrm{J}}$ reduce to

$$
\begin{aligned}
& \mathrm{J}^{\mathrm{t}}=\mathrm{G}+\sigma \mathrm{I}^{\mathrm{t}}, \\
& \mathrm{G}=\frac{16 \mathrm{R} \sqrt{\mu \mathrm{B}_{0} \varepsilon_{\mathrm{r}}}}{\mathrm{M}} \mathrm{kE}\left(\frac{1}{\mathrm{k}}\right) \\
& \mathrm{J}^{\mathrm{r}}=\frac{16 \mathrm{R} \sqrt{\mu \mathrm{B}_{0} \varepsilon_{\mathrm{r}}}}{\mathrm{M}}\left[\mathrm{E}(\mathrm{k})-\left(1-\mathrm{k}^{2}\right) \mathrm{K}(\mathrm{k})\right], \\
& \tilde{\mathrm{J}}^{\mathrm{t}}=\frac{\mathrm{R}_{0}}{\mathrm{R}} \sigma \mathrm{G}, \\
& \tilde{\mathrm{J}}^{\mathrm{r}}=\frac{\mathrm{R}_{0}}{\mathrm{R}} \mathrm{J}^{\mathrm{r}},
\end{aligned}
$$

where $R=R_{0}+\varepsilon_{t} \cos (\theta)$. Note, that the quantity $\mathrm{Jt}$ is an adiabatic invariant only in the case $\frac{1}{\text { qN }} \ll 1$. Substituting Eqs. (19), (20), (28) and (29) into Eq.(18), we finally obtain the bounce averaged kinetic equation for the toroidally and locally trapped particles,

$$
\begin{aligned}
& \frac{\partial J^{\prime}}{\partial \theta} \frac{\partial F}{\partial r}-\frac{\partial J^{\prime}}{\partial r} \frac{\partial F}{\partial \theta}=\sigma \frac{v r \Omega}{B_{0}} \frac{R_{0}}{R} \frac{\partial}{\partial \mu} \mu G \frac{\partial F}{\partial \mu}, \\
& \frac{\partial J^{r}}{\partial \theta} \frac{\partial F}{\partial r}-\frac{\partial J^{r}}{\partial r} \frac{\partial F}{\partial \theta}=\frac{v r \Omega}{B_{0}} \frac{R_{0}}{R} \frac{\partial}{\partial \mu} \mu J^{r} \frac{\partial F}{\partial \mu} .
\end{aligned}
$$

As was shown in [KS] these equation for the transition particles are solved more easily, if we transform from the variables $(r, \theta, \mu)$ to the variables $\left(r_{0}, \theta_{0}, \mu\right)$, where $r_{0}, \theta_{0}$ are the coordinates of the transition point and related to $\mu$ via $[\mathrm{KS}]$,

$$
\begin{gathered}
\frac{\partial \theta_{0}^{\mathrm{r}}}{\partial \mu}=-\frac{\frac{\partial \mathrm{J}^{\mathrm{r}}}{\partial \mu} \frac{\partial \mathrm{k}_{0}^{2}}{\partial \mathrm{r}_{0}}}{\mathrm{D}_{0}^{\mathrm{r}}} \\
\frac{\partial \mathrm{r}_{0}^{\mathrm{r}}}{\partial \mu}=\frac{\frac{\partial \mathrm{J}^{\mathrm{r}}}{\partial \mu} \frac{\partial \mathrm{k}_{0}^{2}}{\partial \theta_{0}}}{\mathrm{D}_{0}^{\mathrm{r}}}
\end{gathered}
$$




$$
\begin{aligned}
& \frac{\partial \theta_{0}^{\mathrm{l}}}{\partial \mu}=-\frac{\frac{\partial \mathrm{k}_{0}^{2}}{\partial \mu}}{\frac{\partial \mathrm{k}_{0}^{2}}{\partial \theta_{0}}} \\
& \frac{\partial \mathrm{r}_{0}^{\mathrm{l}}}{\partial \mu}=\frac{\frac{\partial \mathrm{J}^{\prime}}{\partial \mu}}{\frac{\partial \mathrm{J}_{0}^{\mathrm{t}}}{\partial \mathrm{r}}}
\end{aligned}
$$

where the subscript " 0 " means that the corresponding quantity is taken in the transition point and the quantity $D^{r}$ has the form,

$$
D^{r}=-\frac{8 R_{0} \sqrt{\mu B_{0} \varepsilon_{r}}}{N \varepsilon_{r}} \varepsilon_{r} \sin (\theta)\left[\frac{V^{\prime}}{\mu B_{0}}+\frac{\varepsilon_{r}^{\prime}}{2 \varepsilon_{r}}\right]
$$

so that the function $\mathrm{f}$ given by Eq.(22) can be rewritten,

$$
f=\frac{N q}{2 \pi r \Omega \partial k^{2} / \partial \theta} D^{r} .
$$

Eqs.(30) and (31) in the lowest order in $\varepsilon_{t}$ in coordinates $\left(r_{0}, \theta_{0}, \mu\right)$ become

$$
\begin{aligned}
& \frac{\partial F}{\partial \theta}=-\frac{\sigma v r \Omega}{B_{0} \frac{\partial J^{\prime}}{\partial r}} \frac{d}{d \mu} \mu G \frac{d F}{d \mu}, \\
& \frac{\partial F}{\partial \theta}=-\frac{v r \Omega}{B_{0} \frac{\partial J^{r}}{\partial r}} \frac{d}{d \mu} \mu \mathrm{J}^{r} \frac{d F}{d \mu},
\end{aligned}
$$

where all the quantities are expressed in new variables, in particular,

$$
\frac{\mathrm{d}}{\mathrm{d} \mu}=\frac{\partial \theta_{0}}{\partial \mu} \frac{\partial}{\partial \theta_{0}}+\frac{\partial \mathrm{r}_{0}}{\partial \mu} \frac{\partial}{\partial \mathrm{r}_{0}}
$$

with $\frac{\partial \theta_{0}}{\partial \mu}$ and $\frac{\partial \mathrm{r}_{0}}{\partial \mu}$ given by Eqs. (32), (33) and (34), (35) for Eqs. (38a) and (38b), respectively. 
Let us look for the low collisional solution of Eqs. (38a) and (38b) in the following form,

$$
F\left(r_{0}, \theta_{0}, \theta\right)=F_{M}\left(r_{0}\right)+\bar{\Psi}\left(r_{0}, \theta_{0}\right)+\tilde{\Psi}\left(r_{0}, \theta_{0}, \theta\right),
$$

where $F_{M}(r)$ is the Maxwellian function, $\bar{\Psi}$ is independent on $\theta$ correction, $\tilde{\Psi}$ varies on the magnetic surface. Using the well-known technique [rosenbluth], we find that the lowest (zeroth) in the collision frequency $\tilde{\Psi}$ is zero, while the first order in $v$ solution obeys

$$
\begin{aligned}
& \frac{\partial \tilde{\Psi}}{\partial \theta}=-\sigma \frac{v_{r} \Omega}{B_{0} \frac{\partial \mathrm{J}^{\mathrm{t}}}{\partial \mathrm{r}}} \frac{\partial \theta_{0}^{\mathrm{l}}}{\partial \mu} \frac{\partial}{\partial \theta_{0}} \mu \mathrm{G}\left(\frac{\partial \mathrm{F}_{\mathrm{M}}}{\partial \mathrm{r}_{0}} \frac{\partial \mathrm{r}_{0}^{\mathrm{t}}}{\partial \mu}+\frac{\partial \theta_{0}^{\mathrm{t}}}{\partial \mu} \frac{\partial \bar{\Psi}}{\partial \theta_{0}}\right) \equiv \frac{\hat{\mathrm{C}}_{1}\left(\mathrm{~F}_{\mathrm{M}}+\bar{\Psi}\right)}{\frac{\partial \mathrm{J}^{\mathrm{t}}}{\partial \mathrm{r}}}, \\
& \frac{\partial \tilde{\Psi}}{\partial \theta}=-\frac{v r \Omega}{B_{0} \frac{\partial J^{r}}{\partial r}} \frac{\partial \theta_{0}^{r}}{\partial \mu} \frac{\partial}{\partial \theta_{0}} \mu \mathrm{J}^{r}\left(\frac{\partial F_{M}}{\partial r_{0}} \frac{\partial r_{0}^{r}}{\partial \mu}+\frac{\partial \theta_{0}^{r}}{\partial \mu} \frac{\partial \bar{\Psi}}{\partial \theta_{0}}\right) \equiv \frac{\hat{C}_{r}\left(F_{M}+\bar{\Psi}\right)}{\frac{\partial J^{r}}{\partial r}} .
\end{aligned}
$$

To find the function $\bar{\Psi}$, we should integrate Eqs.(41) and (42) over the transition trajectory,

$$
0=\oint_{\text {trans.otiix }} \frac{\partial \tilde{\Psi}}{\partial \theta} \mathrm{d} \theta=\int_{\mathrm{r}} \frac{\hat{\mathrm{C}}_{\mathrm{r}}\left(\mathrm{F}_{\mathrm{M}}+\bar{\Psi}\right)}{\frac{\partial \mathrm{J}^{\mathrm{r}}}{\partial \mathrm{r}}} \mathrm{d} \theta+(2 \mathrm{~N}+1) \int_{i} \frac{\hat{\mathrm{C}}_{\mathrm{r}}(\bar{\Psi})}{\frac{\partial \mathrm{J}^{\mathrm{t}}}{\partial \mathrm{r}}} \mathrm{d} \theta+\int_{1} \frac{\hat{\mathrm{C}}_{\mathrm{t}}\left(\mathrm{F}_{\mathrm{M}}\right)}{\frac{\partial \mathrm{J}^{\mathrm{t}}}{\partial \mathrm{r}}} \mathrm{d} \theta
$$

Here, $\mathrm{N}$ is the average number of the full circuits of the particle in the toroidally trapped state before it becomes locally trapped, so that for the odd in $\sigma$ combination under the integral we get the factor $(2 \mathrm{~N}+1)$ of the toroidal passes and the factor 1 for the even combination. Thus, since the radial displacement of the toroidally trapped particle is odd, which manifests itself in the oddity of $\frac{\partial \mathrm{r}_{0}^{\mathrm{t}}}{\partial \mu}$ the last integral in Eq.(43) has the factor unity instead of $(2 N+1)$. We can neglect the term $\frac{\hat{C}_{t}\left(F_{M}\right)}{\frac{\partial I^{2}}{\partial r}}$ in comparison with $\frac{\hat{C}_{r}\left(F_{M}\right)}{\frac{\partial J^{r}}{\partial r}}$, since
their ratio has the order, 


$$
\frac{\mathrm{J}^{\mathrm{r}} \frac{\partial \theta_{0}^{\mathrm{r}}}{\partial \mu} \frac{\partial \mathrm{r}_{0}^{\mathrm{r}}}{\partial \mu}}{\mathrm{G} \frac{\partial \theta_{0}^{\mathrm{r}}}{\partial \mu} \frac{\partial \mathrm{r}_{0}^{\mathrm{r}}}{\partial \mu}} \approx\left(\frac{\mathrm{r}}{\rho}\right) \frac{\varepsilon_{\mathrm{t}}}{\varepsilon_{\mathrm{r}}^{3 / 2} \mathrm{r}^{2} / \partial \mathrm{r}}>>1
$$

The factor $(2 \mathrm{~N}+1)$ in Eq.(43) has a meaning of the inversed trapping probability,

$$
2 \mathrm{~N}+1=\mathrm{P}^{-1} \text {, }
$$

so that Eq.(43) yields,

$$
P \int_{r} \frac{\hat{C}_{r}\left(F_{M}+\bar{\Psi}\right)}{\frac{\partial J^{r}}{\partial r}} d \theta+\int_{1} \frac{\hat{C}_{r}(\bar{\Psi})}{\frac{\partial J^{\prime}}{\partial r}} d \theta=0
$$

Note, that in the second integral we perform integration for the majority state. Let us compare the terms,

$$
\begin{aligned}
& P \int_{r} \frac{\hat{C}_{r}(\bar{\Psi})}{\frac{\partial \mathrm{J}^{\mathrm{r}}}{\partial \mathrm{r}} \mathrm{d} \theta}=\frac{\mathrm{f}}{1+\mathrm{f} / 2} \frac{\left(\frac{\partial \theta_{0}^{\mathrm{r}}}{\partial \mu}\right)^{2} \mathrm{~J}^{\mathrm{r}}}{\frac{\partial \mathrm{J}^{\mathrm{r}}}{\partial \mathrm{r}}} \\
& \int_{\mathrm{r}} \frac{\hat{\mathrm{C}}_{\mathrm{r}}(\bar{\Psi})}{\frac{\partial \mathrm{J}^{\mathrm{t}}}{\partial \mathrm{r}} \mathrm{d} \theta}=\frac{\left(\frac{\partial \theta_{0}^{\mathrm{t}}}{\partial \mu}\right)^{2} \mathrm{G}}{\frac{\partial \mathrm{J}^{\mathrm{t}}}{\partial \mathrm{r}}} .
\end{aligned}
$$

Substituting Eqs.(26a), (27), (32), (34), (37) into expressions (47), we find that the ratio of the first term to the second is $\frac{1}{(1+f / 2) \varepsilon_{\mathrm{r}}}>>1$. That permits us to neglect the second term in Eq.(46) which after that yields,

$$
\frac{\partial \bar{\Psi}}{\partial \theta_{0}}=-\frac{\partial \mathrm{F}_{M}}{\partial \mathrm{r}_{0}} \frac{\int_{\mathrm{r}}\left(\frac{\partial \mathrm{r}_{0}^{\mathrm{r}}}{\partial \mu}\right) \mathrm{d} \theta}{\int_{\mathrm{r}}\left(\frac{\partial \theta_{0}^{\mathrm{r}}}{\partial \mu}\right) \mathrm{d} \theta} \approx \mathrm{F}_{\mathrm{M}}^{\prime} \frac{\frac{\partial \mathrm{k}_{0}^{2}}{\partial \theta_{0}}}{\frac{\partial \mathrm{k}_{0}^{2}}{\partial \mathrm{r}_{0}}}
$$

With the help of the following relations, 


$$
\begin{aligned}
& \frac{\partial \mathrm{k}_{0}^{2}}{\partial \mathrm{r}_{0}}=\frac{\varepsilon_{\mathrm{t}}^{\prime}}{2 \varepsilon_{\mathrm{r}}}\left(\gamma+\cos \left(\theta_{0}\right)\right), \\
& \frac{\partial \mathrm{k}_{0}^{2}}{\partial \theta_{0}}=-\frac{\varepsilon_{\mathrm{t}}}{2 \varepsilon_{\mathrm{r}}} \sin \left(\theta_{0}\right),
\end{aligned}
$$

where

$$
\gamma=-\frac{\frac{V^{\prime}}{\mu B_{0}}+\varepsilon_{r}^{\prime}}{\varepsilon_{t}^{\prime}}
$$

the solution yields,

$$
\bar{\Psi}=-\mathrm{rF} F_{M}^{\prime}\left\{\begin{array}{lll}
\int_{0}^{\theta_{0}} d \theta_{0} \frac{\sin \left(\theta_{0}\right)}{\cos \left(\theta_{0}\right)+\gamma} & \text { mjs }=1, & 0 \leq \theta_{0} \leq \pi \\
\int_{\pi}^{\theta_{0}} d \theta_{0} \frac{\sin \left(\theta_{0}\right)}{\cos \left(\theta_{0}\right)+\gamma} & \operatorname{mjs}=-1, & \pi \leq \theta_{0} \leq 2 \pi
\end{array} .\right.
$$

Let us evaluate everything for mjs $=1$, since the bootstrap current should have the same amplitude, but the opposite sign for mjs=-1. Then integrating Eq.(51) gives,

$$
\bar{\Psi}=-\mathrm{rF}_{M}^{\prime} \ln \left|\frac{1+\gamma}{\cos \left(\theta_{0}\right)+\gamma}\right|
$$

The bootstrap current, produced by the transition particles, $j=\oiint \frac{d \theta d \phi}{(2 \pi)^{2}} \int d^{3} v u \bar{\Psi}$, according to Eq.(17), with $\mathrm{f}$ given by Eq.(12), is

$$
j=2 \pi m j s \int d E \int_{0}^{\pi} d \theta_{0} \frac{B_{0}}{\frac{\partial \theta_{0}^{t}}{\partial \mu}} f(E) \Psi .
$$

Using Eq.(34), which gives $\frac{\partial \theta_{0}^{\iota}}{\partial \mu}=-\frac{1}{\mu \varepsilon_{\mathrm{t}} \sin \left(\theta_{0}\right)}$, we can explicitly find,

$$
\mathrm{j}=-2 \pi m j s \varepsilon_{\mathrm{t}} \int \mathrm{dEf}(\mathrm{E}) \mathrm{rF} \mathrm{F}_{M}^{\prime} \mathrm{E}\left[2+\ln \left|\frac{(1+\gamma)^{2}}{1-\gamma^{2}}\right|\right] \approx-\operatorname{mjs} 4 \pi \varepsilon_{\mathrm{t}} \int \mathrm{dEf}(\mathrm{E}) \mathrm{rF} \mathrm{F}_{M}^{\prime} E \begin{cases}1, & |\gamma|<<1 \\ \frac{1}{\gamma}, & |\gamma| \gg>1\end{cases}
$$




$$
j=\frac{3 m j s \varepsilon_{\mathrm{t}} \sqrt{\varepsilon_{\mathrm{r}}} N q v_{\mathrm{T}}^{2}}{\pi \sqrt{2} \Omega\left(e \Phi^{\prime} / T\right)}\left(\frac{\varepsilon_{\mathrm{r}}^{\prime}}{4 \varepsilon_{\mathrm{r}}}\left[\frac{5 N^{\prime}}{N}-\frac{65 T^{\prime}}{\mathrm{T}}\right]+\frac{e \Phi^{\prime}}{\mathrm{T}}\left[\frac{N^{\prime}}{N}-\frac{4 T^{\prime}}{\mathrm{T}}\right]\right), \text { for } \quad \varepsilon_{\mathrm{t}}^{\prime} \ll\left|\frac{e \Phi^{\prime}}{T}+\varepsilon_{\mathrm{r}}^{\prime}\right|
$$

where $v_{T}=\sqrt{\frac{2 T}{m}}$. The rough estimates for the bootstrap current could be obtained from the heuristic formula,

$$
\mathrm{j} \approx \mathrm{N}^{\prime} \Delta \mathrm{rv}_{\mathrm{T}} \mathrm{f} \varepsilon_{\mathrm{t}},
$$

where $f$ gives the relative difference between the toroidally trapped particles moving in different directions, $\varepsilon_{\mathrm{t}}$ gives the fraction of the toroidally trapped particles in $\mu$ space, $\Delta r$ is the radial width of the transition particle which is determined by the radial displacement of the particle in the locally trapped state,

$$
\Delta r \approx \frac{\varepsilon_{\mathrm{t}}}{\max \left\{\frac{\mathrm{e} \Phi^{\prime}}{\mathrm{T}}, \varepsilon_{\mathrm{t}}^{\prime}, \varepsilon_{\mathrm{r}}^{\prime}\right\}}
$$

For comparison, let us recall the bootstrap current in the usual banana regime,

$$
\mathrm{j}_{\mathrm{B}} \approx \mathrm{N}^{\prime} \Delta \mathrm{r}_{\mathrm{B}} \mathrm{v}_{\mathrm{T}} \approx\left(\frac{\mathrm{q} \rho}{\sqrt{\varepsilon_{\mathrm{t}}}}\right) \mathrm{N}^{\prime} \mathrm{v}_{\mathrm{T}}
$$

with $\Delta \mathrm{r}_{\mathrm{B}}$ being the displacement of the toroidally trapped particles, but since the current is produced by the passing particles, there is no any geometrical factor, like $\varepsilon_{\mathrm{t}}$ in Eq.(57). 


\section{References}

1. R.J. Bickerton, J.W. Connor, J.B. Taylor, Nature Phys. Sci. 229, 110 (1971).

2. L.M. Kovrizhnykh, Nuclear Fusion 24, 851 (1984).

3. J.W. Connor, J.B. Taylor, Comments Plasma Phys. Controlled Fusion 11, 37 (1987).

4. A.H. Boozer, H.J. Gardner, Phys. Fluids B2, 2408 (1990).

5. M.N. Rosenbluth, R.H. Haseltine, F.L. Hinton, Phys. Fluids 15, 112 (1972).

6. A. Pytte, A.H. Boozer, Phys. Fluids 24, 88 (1981).

7. K.C. Shaing, E.C. Crume, Jr., J.S. Tolliver, S.P. Hirshman, W.J. van Rij, Phys. Fluids B1, 148 (1989);

K.C. Shaing, J.D. Callen, Phys. Fluids 26, 3315 (1983).

8. John R. Cary, S.G. Shasharina, Submitted to Phys. Fluids.

9. L.M. Kovrizhnykh, S.G. Shasharina, Nuclear Fusion 30, 453 (1990). 


\section{Figure Captions}

Fig.1 Transition orbit for the case, when "-"-state is a majority state. In the point "T", the trapping occurs with the probabilty $\mathrm{P}$, the reflection occurs with the probability (1-P).

Fig.2 Transition orbit for the case, when " $r$ "-state is a majority state.

Fig.3a The phase portrait of the trapping processe. Thick dashed lines denote the sparatrices between the locally and toroidally trapped states. Thick solid lines represent the trajectories. The trapping particles are limited by the trajectory $\mathrm{B}$ and the point $\mathrm{A}$. The incident unperturbed flux is limited by $A$ and $B$, the perturbed flux is limited by $A$ and D.

Fig.3b The same processe as on Fig.3a, but in $(\mu B, \phi)$ space. 

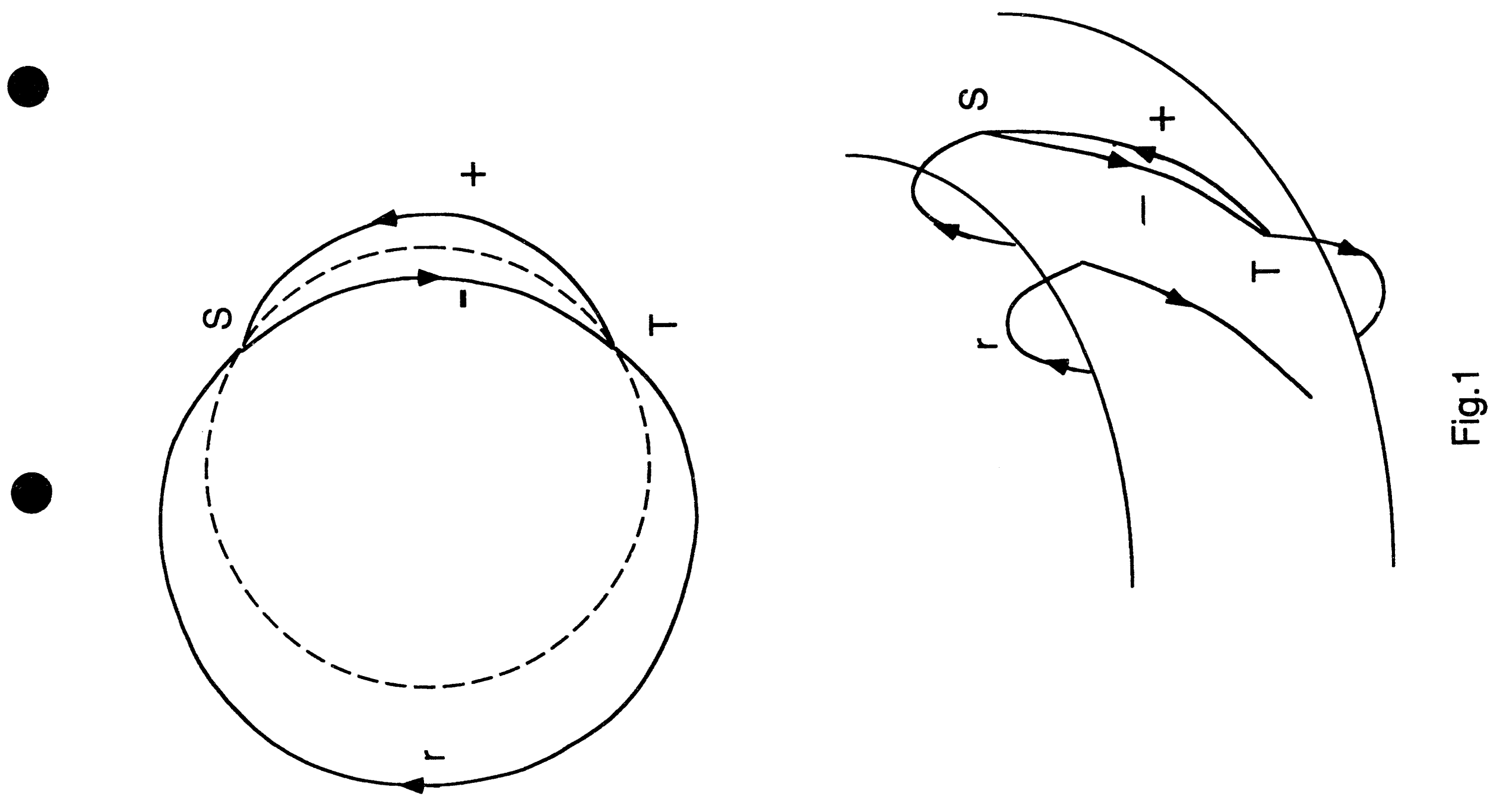

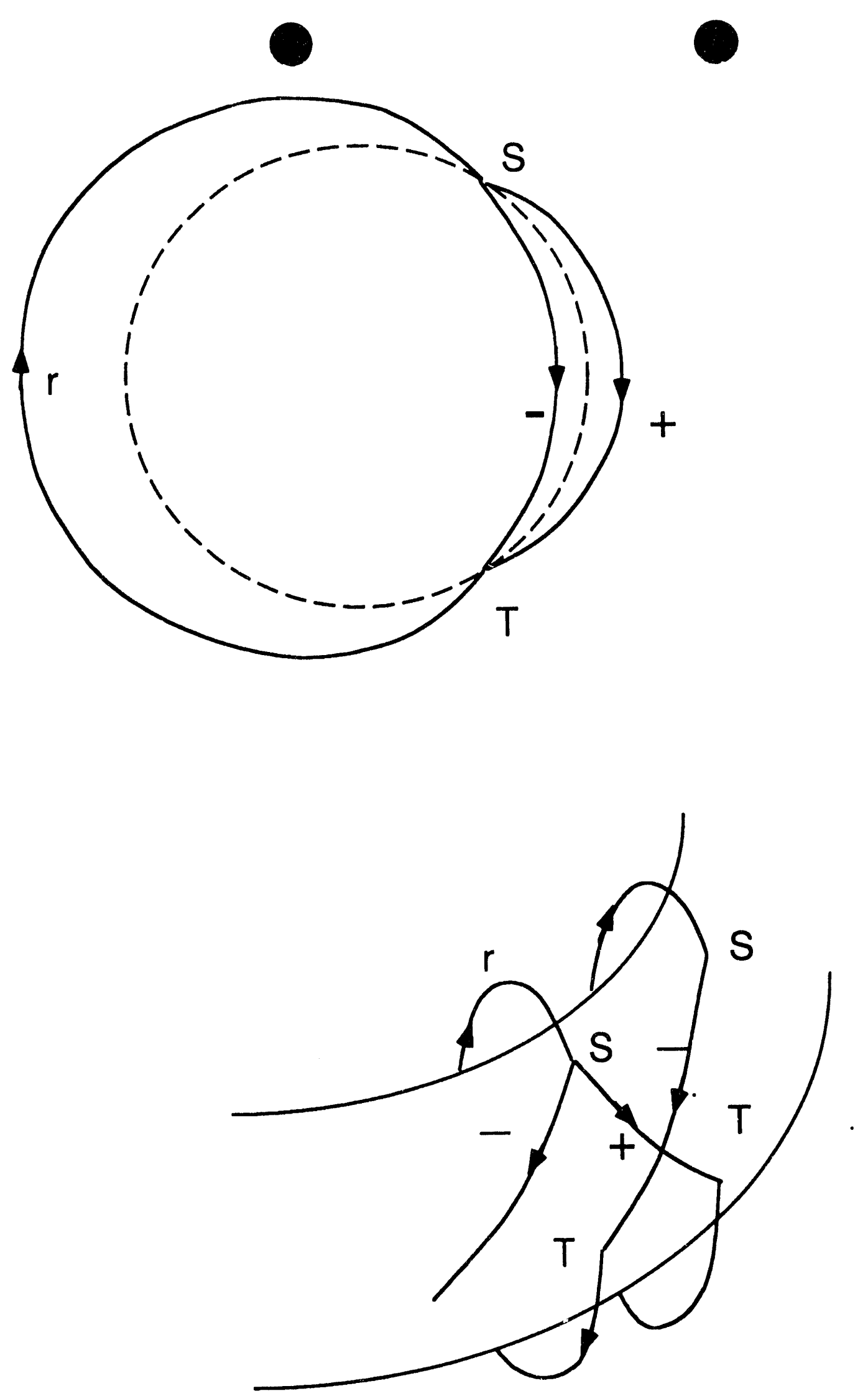

Fig.2 


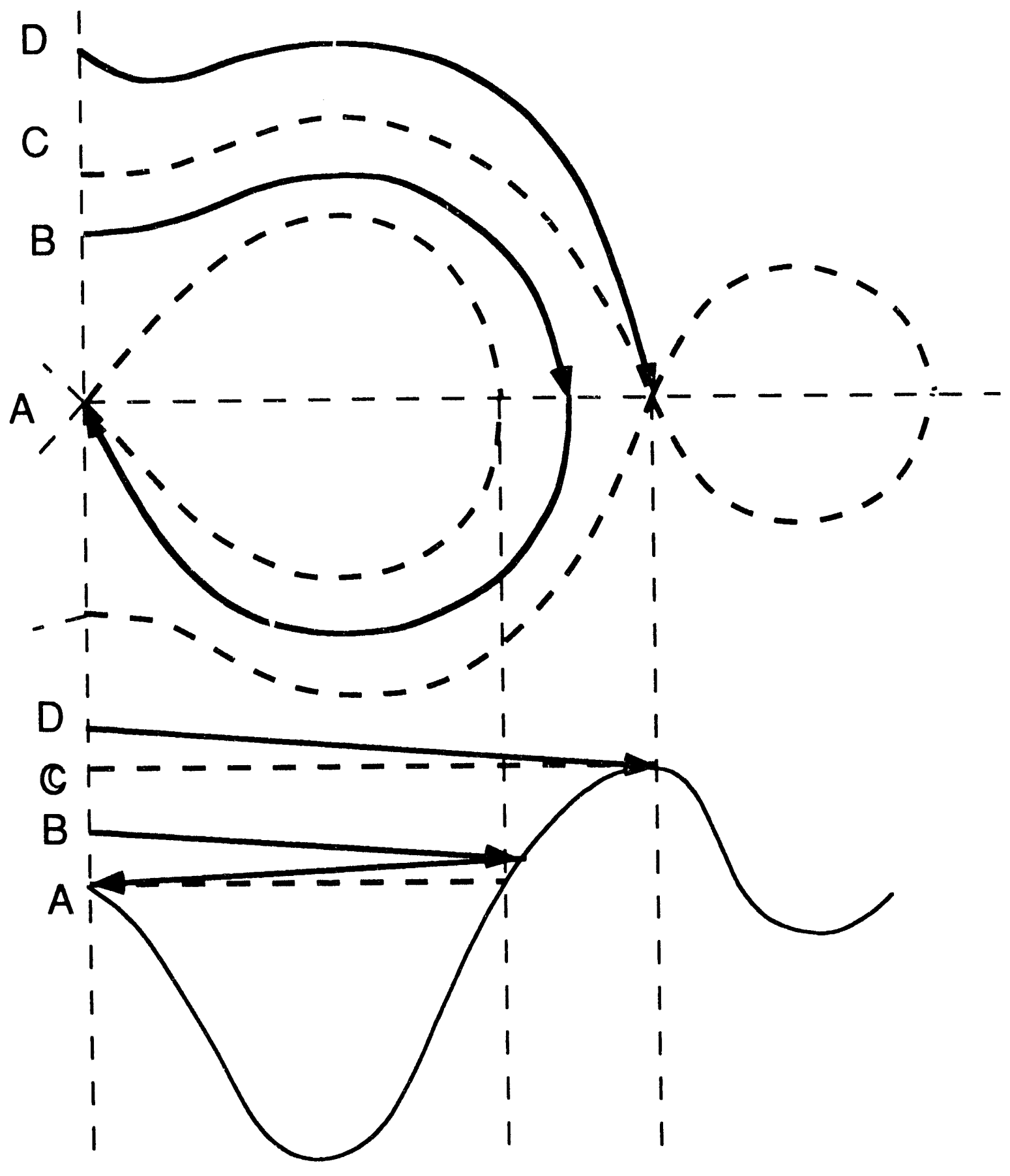

Fig.3(a,b) 

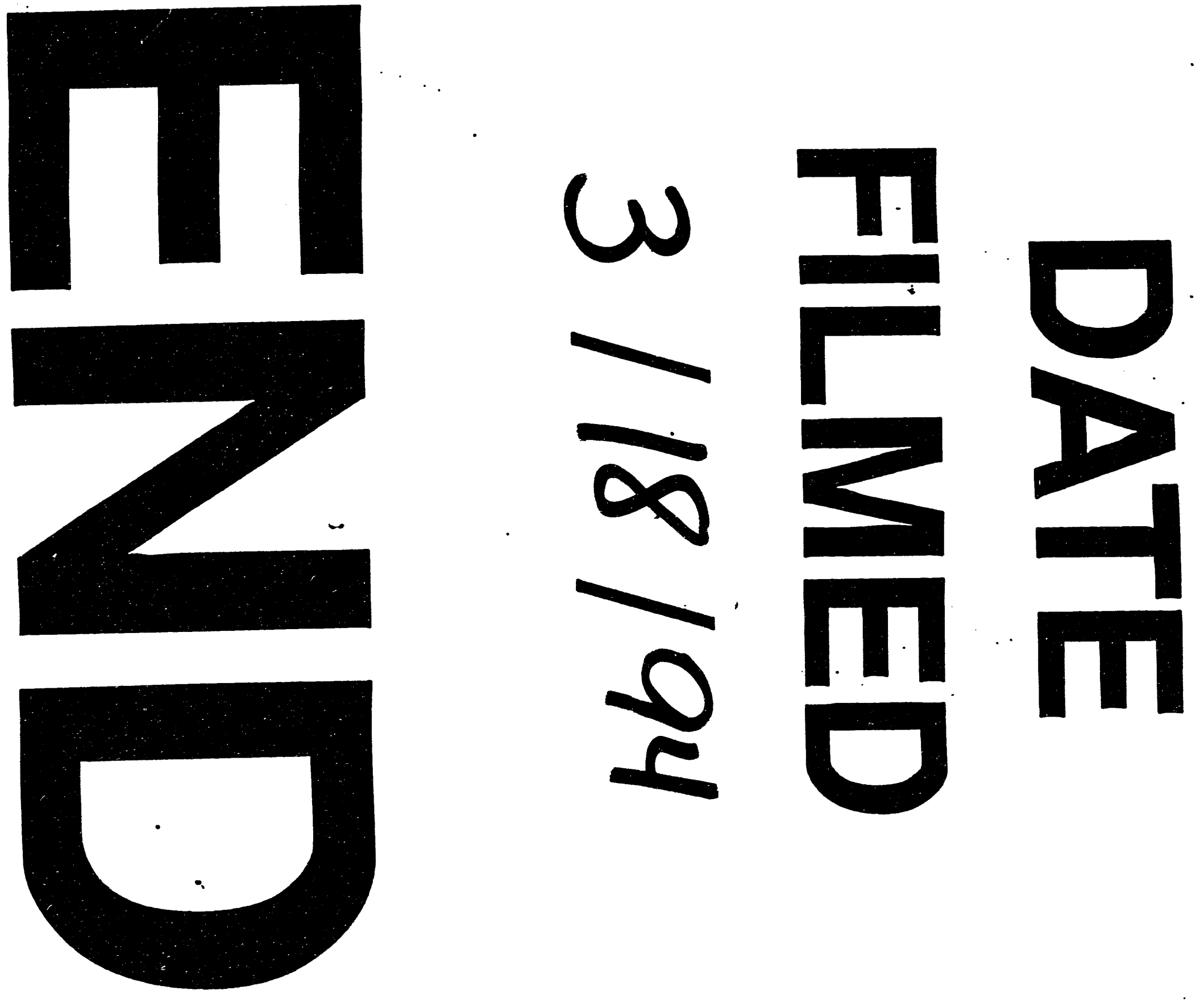


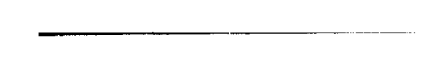

